\title{
DESIGN OPTIMIZATION OF AIRCRAFT LANDING GEAR ASSEMBLY UNDER DYNAMIC LOADING
}

by

Jonathan Y. B. Wong

A thesis submitted to the Department of Mechanical \& Materials Engineering

In conformity with the requirements for

the degree of Master of Applied Science

\author{
Queen's University \\ Kingston, Ontario, Canada
}

(September, 2017)

Copyright (C) Jonathan Y. B. Wong, 2017 


\begin{abstract}
As development cycles and prototyping iterations begin to decrease in the aerospace industry, it is important to develop and improve practical methodologies to meet all design metrics. This research presents an efficient methodology that applies high-fidelity multi-disciplinary design optimization techniques to commercial landing gear assemblies, for weight reduction, cost savings, and structural performance dynamic loading. Specifically, a slave link subassembly was selected as the candidate to explore the feasibility of this methodology. The design optimization process utilized in this research was sectioned into three main stages: setup, optimization, and redesign.

The first stage involved the creation and characterization of the models used throughout this research. The slave link assembly was modelled with a simplified landing gear test, replicating the behavior of the physical system. Through extensive review of the literature and collaboration with Safran Landing Systems, dynamic and structural behavior for the system were characterized and defined mathematically. Once defined, the characterized behaviors for the slave link assembly were then used to conduct a MultiBody Dynamic (MBD) analysis to determine the dynamic and structural response of the system. These responses were then utilized in a topology optimization through the use of the Equivalent Static Load Method (ESLM). The results of the optimization were interpreted and later used to generate improved designs in terms of weight, cost, and structural performance under dynamic loading in stage three. The optimized designs were then validated using the model created for the MBD analysis of the baseline design.

The design generation process employed two different approaches for post-processing the topology results produced. The first approach implemented a close replication of the topology results, resulting in a design with an overall peak stress increase of $74 \%$, weight savings of $67 \%$, and no apparent cost savings due to complex features present in the design. The second design approach focused on realizing reciprocating benefits for cost and weight savings. As a result, this design was able to achieve an overall peak stress increase of $6 \%$, weight and cost savings of $36 \%$, and $60 \%$, respectively.
\end{abstract}




\section{Acknowledgements}

I would like to take this time to thank my supervisor Dr. Il-Yong Kim for providing me with the opportunity and resources to excel in my professional development. His expertise, guidance, and meticulous work mentality has inspired me to achieve greatness beyond my own contemplation. For this, I am truly grateful for everything you have done to help me change for the better as both an individual as well as an engineer.

To both current and past students of the Structural and Multidisciplinary Systems Design Group, thank you all for your selfless support to help me persevere over the challenges I have faced. In particular, many thanks to my fellow Calgarian Luke Ryan for always being a voice of reason that have turned some of my crazy ideas into feasible and successful outcomes. I genuinely could not have done this Masters without your expertise (and sarcasm!).

Thank you to my family for their unquestionable love, moral support, and motivation that has led me to experience the brightest of days and overcome the darkest of hours throughout my life. Although you may no longer be with us, I want to also take this opportunity express my deep gratitude to Norman Chin, for aspiring me to become who I am today. I sincerely thank you for being the big brother I always needed but never deserved.

To the staff and professors of Queen's University, thanks for the help and support you have provided for the past six years. An especially big thank you to Gabrielle Whan for just being someone I could always count on.

Finally, thank you to Safran Landing Systems and the National Science and Engineering Research Council of Canada for providing me with this unique and exciting opportunity as a research project. I would like to personally thank Joseph Lan and James Ning for their amazing support and advice throughout this project. It was an amazing experience and your contributions to it are greatly appreciated. 


\section{Table of Contents}

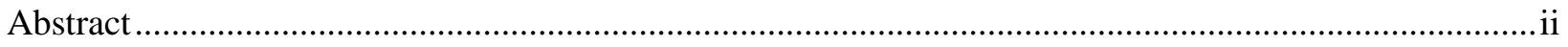

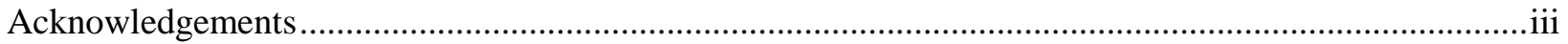

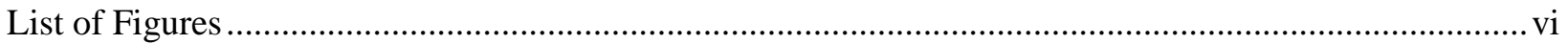

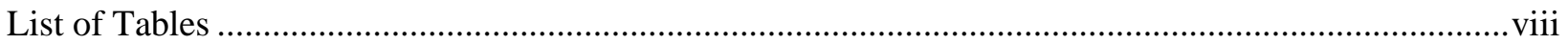

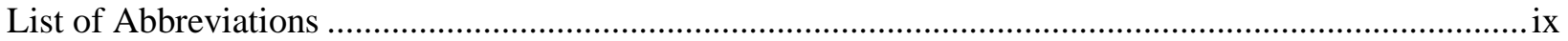

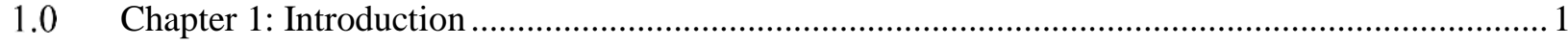

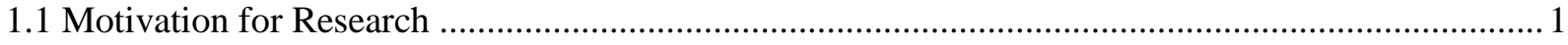

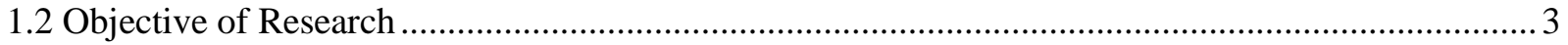

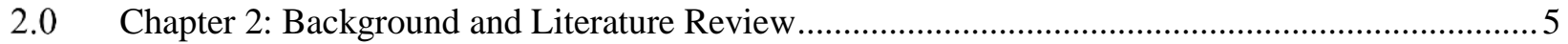

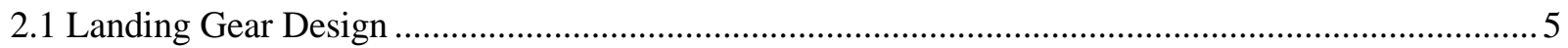

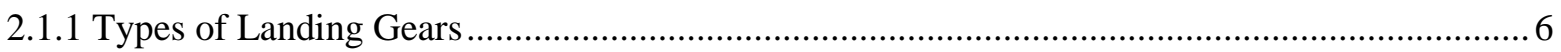

2.1.2 Suspension Configurations for Landing Gears .................................................................. 7

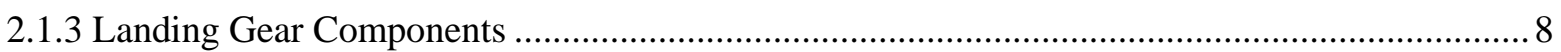

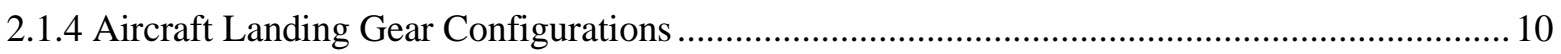

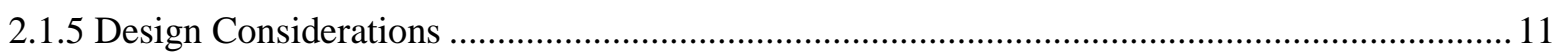

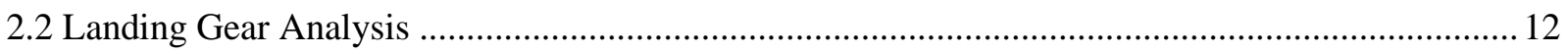

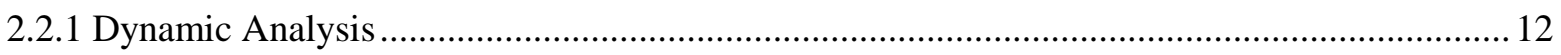

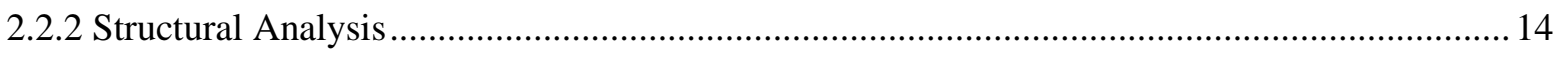

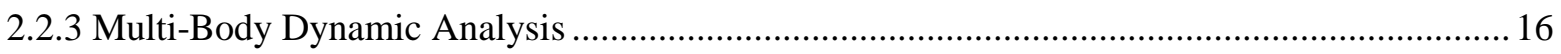

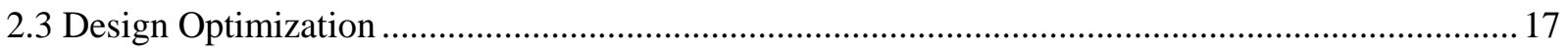

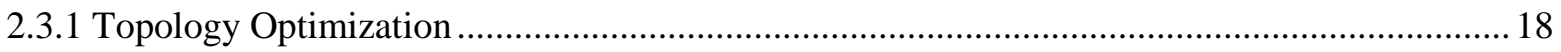

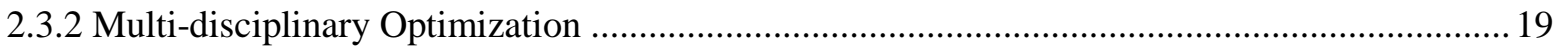

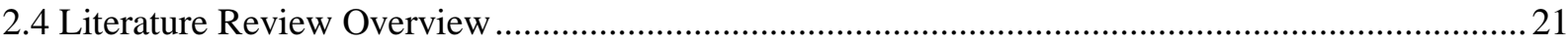

3.0 Chapter 3: Design Optimization Process ..............................................................................22

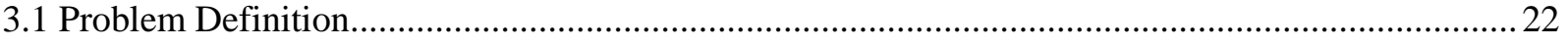

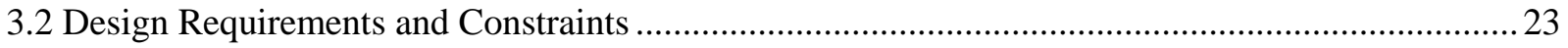

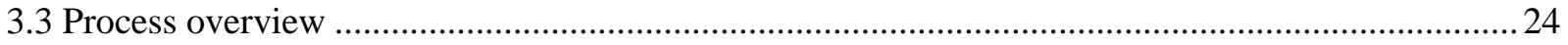

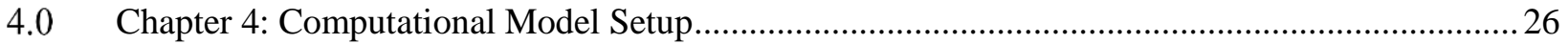

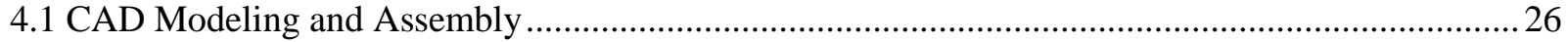

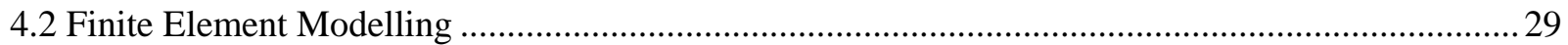

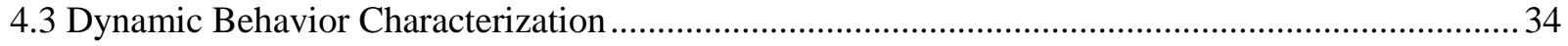

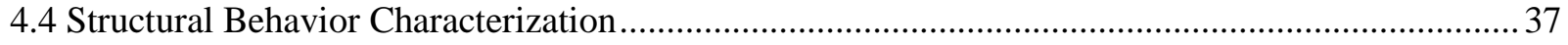




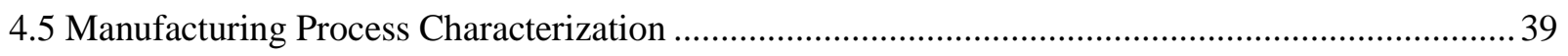

5.0 Chapter 5: Design Analysis and Optimization........................................................................ 42

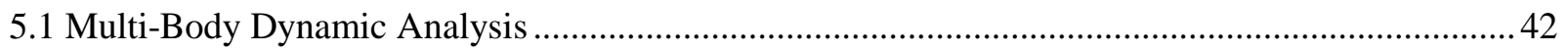

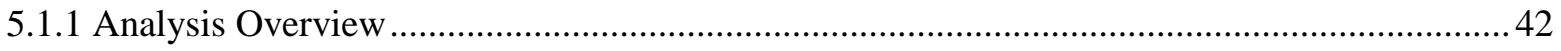

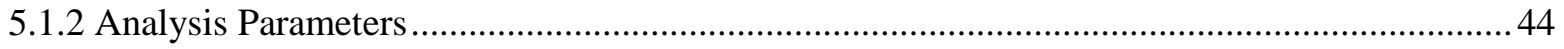

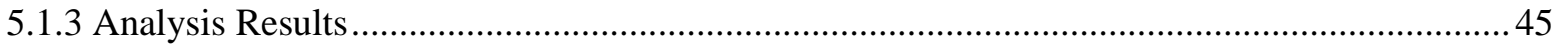

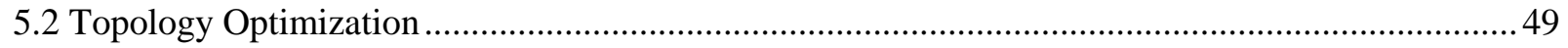

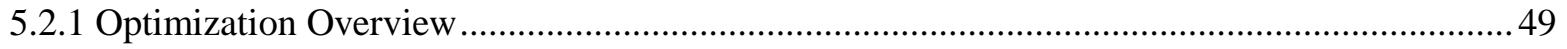

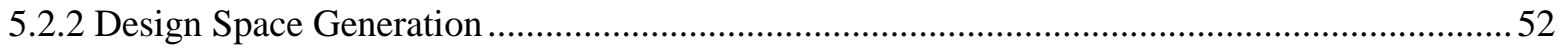

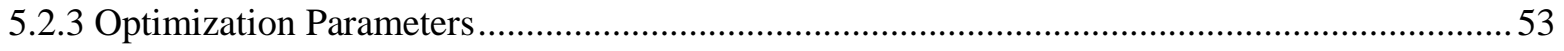

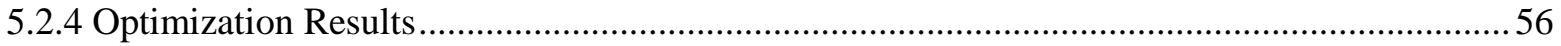

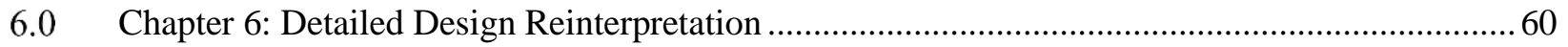

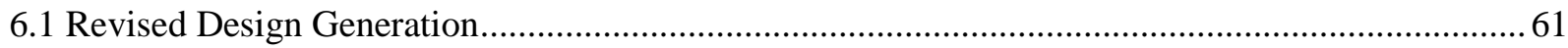

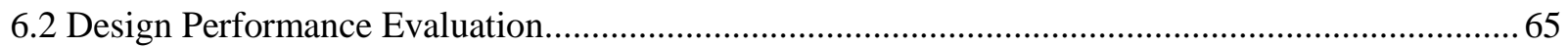

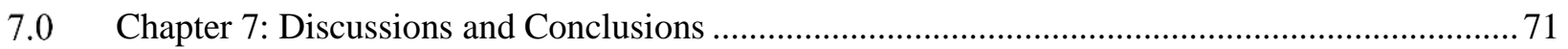

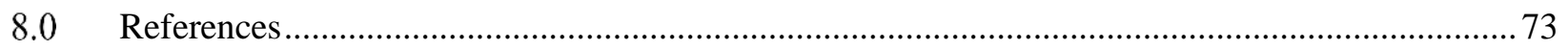

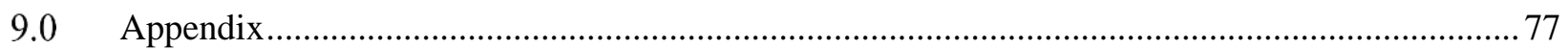

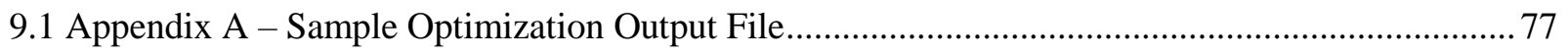

9.2 Appendix B - MATLAB Output File Parser Code ................................................................... 78

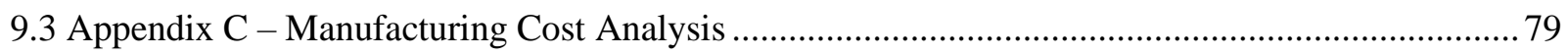

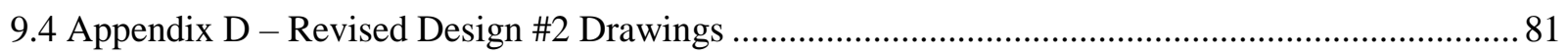




\section{List of Figures}

Figure 1 - Fixed (Left) versus Retractable (Right) landing gear system [10][12] ....................... 6 Figure 2 - Various landing gear strut and shock absorber configurations [10]. The telescopic configuration is by far the most common in commercial aviation and will be the focus for this research, however other configurations are still used today in specific applications. ..................... 7 Figure 3 - List of components typically found in most commercial aircraft with a telescopic and retractable landing gear system [13]. Key components have labels outlined in red and will be

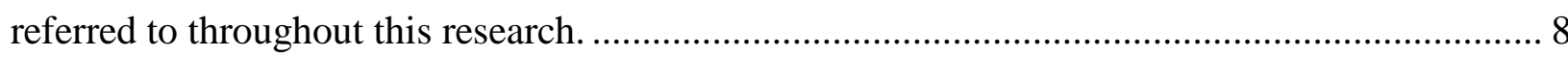
Figure 4 - (A) Boeing 747 - Main and backup lines through the torque link, (B) Boeing 777 Both lines going through torque link and a slave link, (C) Superjet 100 - Backup lines through the slave link and main lines through the torque link. [14] [15] [16] .......................................... 9 Figure 5 - Various aircraft landing gear configurations of different models. [18]....................... 10 Figure 6 - Shimmy (left) and Gear Walk (right) vibrational load phenomena [26]..................... 13 Figure 7 -2D Topology Optimization Example. Design domain, fixed boundary, and point load is defined (left) and the resulting optimized structure (right) [39].............................................. 18 Figure 8 - Diagram outlining the ESLM process.[46] ........................................................... 20 Figure 9 - Slave link assembly for a Sukhoi Superjet 100 aircraft. [16] ..................................... 22 Figure 10 - Slave link assembly design optimization process overview...................................... 24

Figure 11 - Simplified upper slave link drawing (left) and CAD model (right). ......................... 26

Figure 12 - Simpified lower slave link drawing (left) and CAD model (right). ........................... 27

Figure 13 - Simplified upper cable guide drawing (left) and CAD model (right). ...................... 27 Figure 14 - Simplified lower cable guide drawing (left) and CAD model (right). ...................... 28 Figure 15 - Slave link assembly components on a simplified landing gear CAD Model. Design optimization was applied to the upper slave link and lower slave link. ...................................... 29 Figure 16 - Finite element model of slave link assembly on simplified landing gear test rig. .... 30 Figure 17 - Joint definitions available in Altair Hyperworks and utilized in the model............... 30

Figure 18 - Joint modeling techniques and effects on analysis............................................... 32

Figure 19 - Translational input motion $\boldsymbol{u t}$ applied to the cylindrical number J2......................... 34

Figure 20 - Rotational Shimmy motion $\boldsymbol{\theta y t}$ applied to the cylindrical joint J2 ........................ 35

Figure 21 - Rotational gear walk motion $\boldsymbol{\theta z t}$ applied to the revolute joint $\mathrm{J} 1$........................... 35 


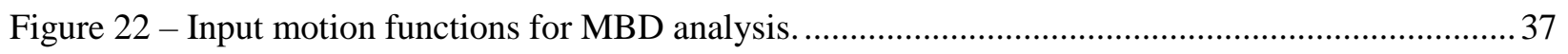

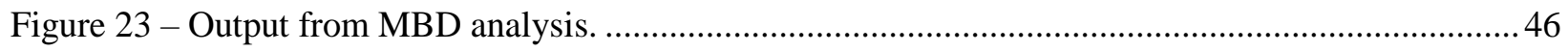

Figure 24 - Stress contours for baseline slave link components at $\mathrm{t}=0.008$ s.................................... 47

Figure 25 - Stress contours for slave link components for various time steps. .......................................48

Figure 26 - MBD topology optimization overview flowchart.............................................................51

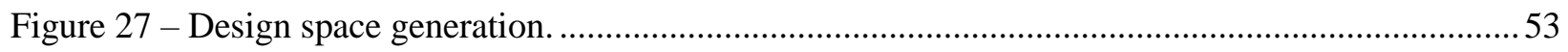

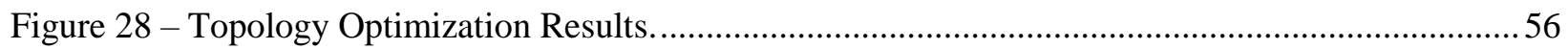

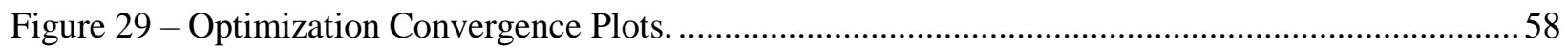

Figure 30 - Abrupt changes in compliance due to ESLM outerloop recalculation of loads .....................59

Figure 31 - RD\#1 interpreted from topology isosurface results ...........................................................62

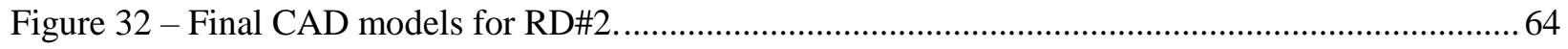

Figure 33 - Reconfigured finite element models for the revised designs..............................................66

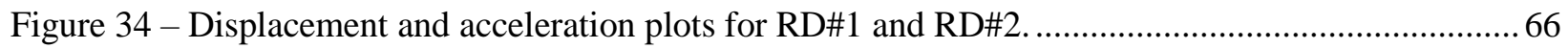

Figure 35 - Stress contours and plots comparison for revised and baseline designs..............................67

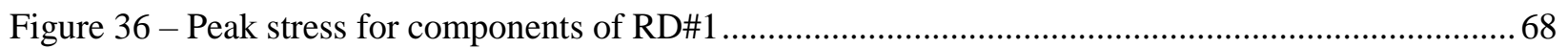

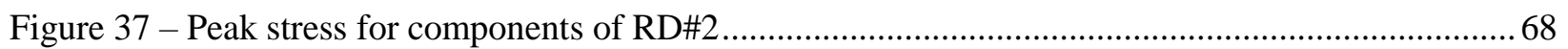

Figure A-38 - Revised Design \#2 lower slave link detailed drawing.................................................... 81

Figure A-39 - Revised Design \#2 upper slave link detailed drawing ................................................... 82 


\section{List of Tables}

Table 1 - Unit Consistency for the slave link landing gear model ......................................................29

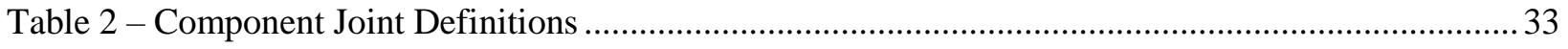

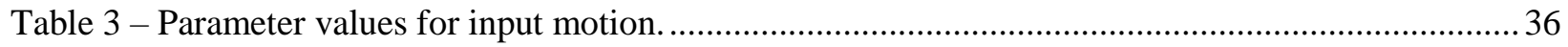

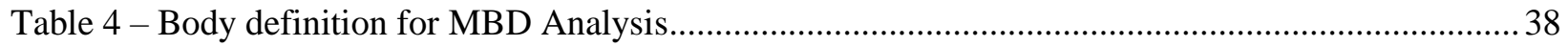

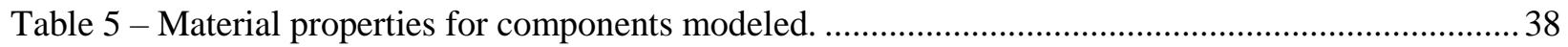

Table 6 - Manufacturing cost model metrics and weight factors ........................................................ 41

Table 7 - Optimization parameters, settings, and computational cost..................................................55

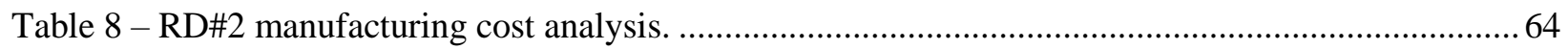

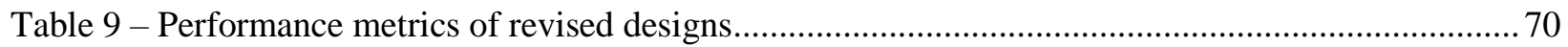

Table A-10 - Manufacturing cost analysis for upper slave link.......................................................... 79

Table A-11 - Manufacturing cost analysis for upper slave link. .......................................................... 80 


\section{List of Abbreviations}

\begin{tabular}{|c|c|}
\hline Abbreviation & Definition \\
\hline MBD & Multi-Body Dynamic \\
\hline ESLM & Equivalent Static Load Method \\
\hline IATA & International Air Transport Association \\
\hline OEW & Operating Empty Weight \\
\hline MTOW & Maximum Takeoff Weight \\
\hline NLG & Nose Landing Gear \\
\hline MLG & Main Landing Gear \\
\hline CAD & Computer Aided Design \\
\hline FEA & Finite Element Analysis \\
\hline FAA & Federal Aviation Administration \\
\hline MMA & Methods of Moving Asymptotes \\
\hline USL & Upper Slave Link \\
\hline LSL & Lower Slave Link \\
\hline $\mathrm{CNC}$ & Computer Numerical Control \\
\hline DOF & Degree Of Freedom \\
\hline TRANS & Translational \\
\hline ROT & Rotational \\
\hline DAE & Differential Algebraic Equation \\
\hline $\mathrm{BDF}$ & Backward Differentiation Formula \\
\hline CMS & Component Mode Synthesis \\
\hline CPU & Central Processing Unit \\
\hline $\mathrm{CG}$ & Center of Gravity \\
\hline SIMP & Solid Isotropic Microstructure Penalization \\
\hline MINLP & Mixed Integer Non-Linear Problem \\
\hline STL & Stereo Lithography \\
\hline RD\#1 & Revised Design \#1 \\
\hline $\mathrm{RD \# 2}$ & Revised Design \#2 \\
\hline
\end{tabular}




\subsection{Chapter 1: Introduction}

The continued strive towards advancing lightweight and cost reduction design practices in the aerospace industry has promoted collaboration between industry and academic institutions. In conjunction with Safran Landing Systems, Queen's University has developed a methodology to computationally analyze and optimize both dynamic and structural aspects of landing gear assemblies. The goal of implementing this methodology was to reduce the weight and cost of landing gear components while satisfying structural performance requirements in dynamic loading scenarios.

\subsection{Motivation for Research}

As our global interactions continue to expand, there will be a subsequent increase in demand for aviation as the mode of transportation to accommodate this growth. The International Air Transport Association (IATA) forecasts a doubling of annual air travelers over the next 20 years from approximately 3.8 billion passengers in 2016 to 7.2 billion in 2035 [1]. In order to meet these demands, airline companies will need to expand their fleets of aircraft in order to grow their network of operations. Although these demands continue to incur an increase in air traffic, it is also equally important to consider the environmental impacts of a growing aviation industry. To offset these impacts, IATA has set a target of an average improvement of $1.5 \%$ in fuel efficiency per year from 2009 to 2020 [1]. The use of lightweight design techniques on aircraft components may be utilized to improve fuel efficiency. It has been shown that for 10 kilogram of weight savings, 3925 kilograms of fuel and subsequently 4 tons of $\mathrm{CO}_{2}$ can be saved [2].

In response to weight saving incentives, the aerospace industry has taken initiatives in various areas to realize these benefits. The weight distribution of a typical aircraft can be broken down into three main categories: total fuel load, total payload, and operating empty weight (OEW) of the aircraft. Within each of these categories lies unique design challenges, which have led to some innovative solutions. 
Fuel management is vital to ensure aircrafts can reach their destination with a margin of safety in reserves, however it is also important to note that fuel contributes approximately $20 \%$ to $30 \%$ of a typical commercial aircraft's maximum takeoff weight (MTOW)[3]. To put this into perspective, a Boeing 777-300ER burns roughly 114 tons of fuel in a one-way flight from Hong Kong to Toronto [4]. This has consequently driven a strong interest into improving aircraft engine technology for better fuel efficiency, with the results being consistently realized for the past 50 years [5]. It has also driven a rise in innovative solutions such as electrification of the landing gear system to reduce taxi and idle fuel consumption.

Although the payload contributes the least to the MTOW in most cases, approximately $20 \%$ [3], it still represents an important area for the aerospace industry to optimize. Unlike the other two categories, the percentage contribution of the payload to the MTOW has the highest variability and plays a key role in determining the economic feasibility for a particular flight. One of the methods implemented to help mitigate this variability are baggage weight restrictions imposed by most airline companies [6]. Apart from this and other logistical solutions, research into introducing weight savings for the payload is limited.

The remaining $50 \%$ to $60 \%$ of the MTOW is from the OEW of an aircraft and is the main area of focus for introducing weight savings. Since an aircraft is comprised of many different components and subsystems, there is a vast amount of research going into various aspects and parts of aircraft design [7][8]. Although there have been significant advances in this field, there are still systems where introducing weight savings may prove to be difficult due to the inherent complexities associated with said system. An example of these challenging systems to optimize includes aircraft landing gears.

Aircraft landing gear systems are composed of numerous actuation mechanisms and structural bracing components interconnected together. Their function is to enable movement while on the ground, and improve aerodynamic efficiency by being stowed away during flight. Due to weight and space limitations, few redundancy features exist within landing gear systems. However, 
they must endure the extreme impact and vibrational loading experienced during landing and braking, and are consequently deemed critical components. These aspects are in essence some of the main challenges that come with landing gear design. Additionally, it is also imperative that these components are designed with a high degree of robustness and reliability. As the aerospace industry continues to shorten their development cycles and number of prototyping iterations while setting more ambitious cost and weight targets, it is important to develop and improve practical, efficient, and accurate design methodologies to meet these growing demands and safety requirements.

\subsection{Objective of Research}

The objective of this research was to develop and perform high-fidelity multi-disciplinary design optimization of commercial landing gear assemblies for weight, cost, and performance by considering structural and dynamic behaviours. Specifically, a slave link subassembly would be selected as the candidate to explore the feasibility of this methodology. The components considered are tertiary, which do not require expensive and time-consuming recertification testing in order to be put into service and thus have a greater potential of being implemented, if proven successful. The sole purpose of the slave link subassembly is to guide secondary hydraulic and electrical lines from the upper portion landing gear to the wheel hub. An overview of the methodology is summarized as follow:

1. Utilize finite element methods to efficiently analyze structural and dynamic aspects of a landing gear assembly under dynamic loading scenarios

2. Perform design optimization to minimize the weight and cost of targeted landing gear components while satisfying performance requirements

3. Generate several lightweight and low cost revised designs based on the findings from the design optimization 
4. Perform trade-off studies for revised designs with the baseline design to obtain design insights and recommendations

Further details pertaining to the proposed methodology are elaborated in Section 3.0. This research stands to have a significant impact in the aerospace industry as it has the potential to drastically reduce design development time and prototyping iterations by optimizing structural and dynamic aspects of a system at the computational stage. Although the methodology presented in this research focuses on a specific application to landing gear systems, it should be noted that it can be applied to the design of other aircraft systems or even other industries. 


\subsection{Chapter 2: Background and Literature Review}

The literature review presented in this research gives an overview for how design in the aerospace industry has been done in the past along with emerging ideas, trends, and technology to improve on existing practices. It is also important to note there are many aspects that go into detailed landing gear design, which are not all extensively discussed in this research. Most of these aspects will be mentioned in a high-level overview in order to support a key conclusion that has been drawn from this review: although there has been a wealth of research done on different aspects of landing gear design independently, there has been very limited work done that considers multiple aspects simultaneously. This is an emerging trend which the aerospace industry is very interested in pursuing. The philosophy behind utilizing multi-disciplinary design solutions as opposed to traditional design practices stems from the belief that each aspect has an effect on the other and optimizing for one aspect of the design may not lead to a design that is optimal in another aspect. Therefore, the goal of this research was to realize this trend by optimizing several components considering both dynamic and structural aspects. Other than a white paper published by Altair Engineering on the potential to utilize this technique for landing gear systems, which does not provide any concrete methodologies or results [9], there has been no mention of any landing gear assembly optimization that considered dynamic and structural aspects simultaneously. Therefore, this research is likely the first attempt at establishing a repeatable and innovative methodology for dynamic and structural optimization for landing gear assemblies.

\subsection{Landing Gear Design}

One of the most important and critical subsystems in an aircraft is the landing gear, as it enables aircrafts to gain enough velocity while on the ground to achieve takeoff as well as ensure a safe and smooth touchdown when the destination is reached. Despite being such a heavily loaded structure, this subsystem typically makes up only $2.5 \%$ to $5 \%$ of the weight of an aircraft and roughly $1.5 \%$ of the overall initial sunk cost [10]. However, it has been determined that 
approximately $50 \%$ of the total damages that incur on a commercial aircraft over its lifetime are related to taxi, take-offs and landing, which correspond to $20 \%$ of the direct maintenance cost [10][11]. Given these facts, one can start to see some of the challenges that designers may face when trying to developing improvements to the existing system. In order to gain a better understanding of these challenges, a basis of the fundamentals of landing gear design must be established, and will be covered in the following subsections.

\subsubsection{Types of Landing Gears}

The landing gear system is analogous with the wheel, brake, steering, and suspension system of an automobile in that they serve the purpose of enabling movement and maneuvering. A key difference between the aerospace and automotive system is landing gears may have the additional functionality of being either fixed or retractable. There are advantages and disadvantages to both types of landing gears. Fixed landing gears benefit from being simple and consequently very cost efficient relative to retractable systems. However, they typically suffer in terms of aerodynamic performance due to the drag generated by the landing gear protrusion. Retractable landing gears have the benefit of reducing drag by allowing the system to be blended into the body of the airframe, however, these systems are usually more costly due to the added complexities associated with the additional components required to enable the retraction mechanism. An example of each system is shown in Figure 1.
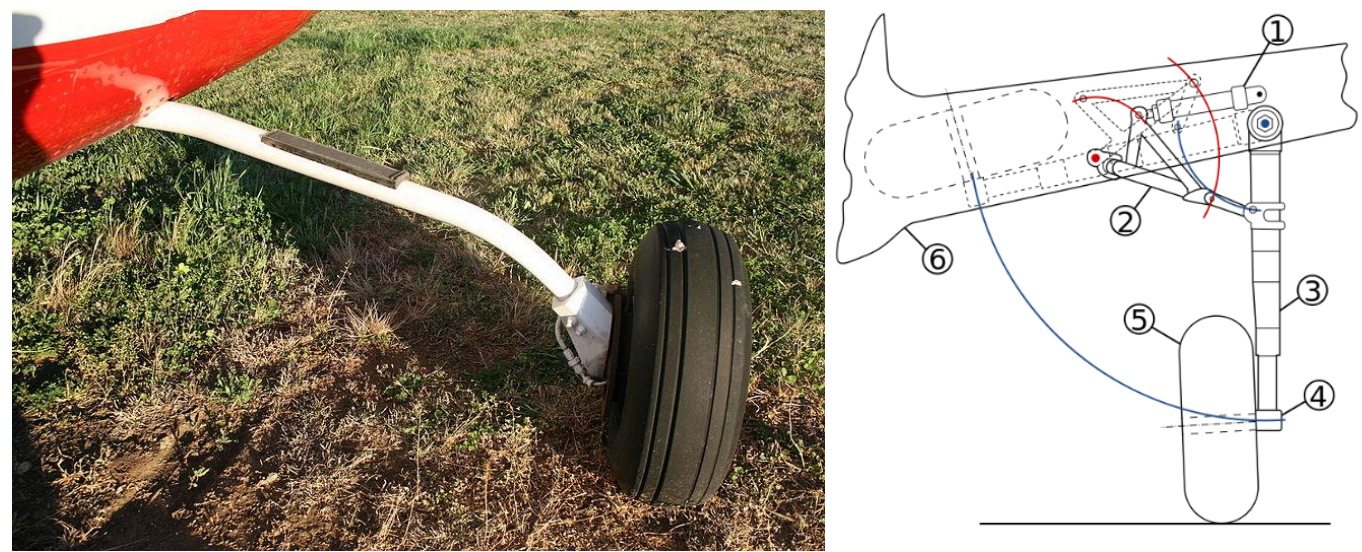

Figure 1 - Fixed (Left) versus Retractable (Right) landing gear system [10][12]. 
Generally speaking, fixed landing gears are more prevalent in smaller and low speed aircraft because the cost incentives outweigh the loss of aerodynamic performance, which is relatively minor in these circumstances. Retractable landing gear systems are more popular in commercial and military applications, where aerodynamic performance significantly outweighs the cost of implementing the retraction mechanism. From a structural and dynamic perspective, the roots for one of the first challenges in landing gear design comes from the use of retractable landing gear systems, and will be further discussed in Section 2.2.

\subsubsection{Suspension Configurations for Landing Gears}

The suspension system plays an essential role in mitigating and dampening the harsh loading, which the landing gear system must endure during take-off and touchdown. By converting the kinematic and potential energy from ground events into a form used for displacing and subsequently heating a fluid medium within a shock absorber, the suspension system is able to dissipate $85 \%$ to $90 \%$ of the total energy generated [11]. Although fundamentals behind the suspension system are the same for all aircraft, there are multiple approaches and designs for achieving this functionality. These configurations are shown in Figure 2.

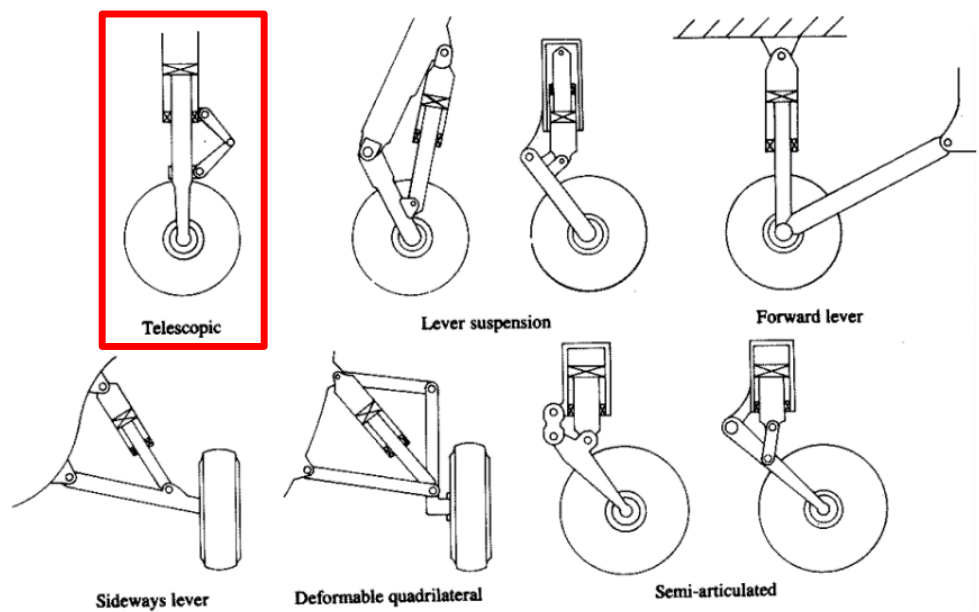

Figure 2 - Various landing gear strut and shock absorber configurations [10]. The telescopic configuration is by far the most common in commercial aviation and will be the focus for this research, however other configurations are still used today in specific applications. 


\subsubsection{Landing Gear Components}

In order to gain a better understanding of the landing gear design process, the nomenclature typically used to identify each landing gear component and its functionality should be defined. A generic retractable and telescopic landing gear system is shown below in Figure 3. Note that certain features and components may vary from one aircraft to the other.

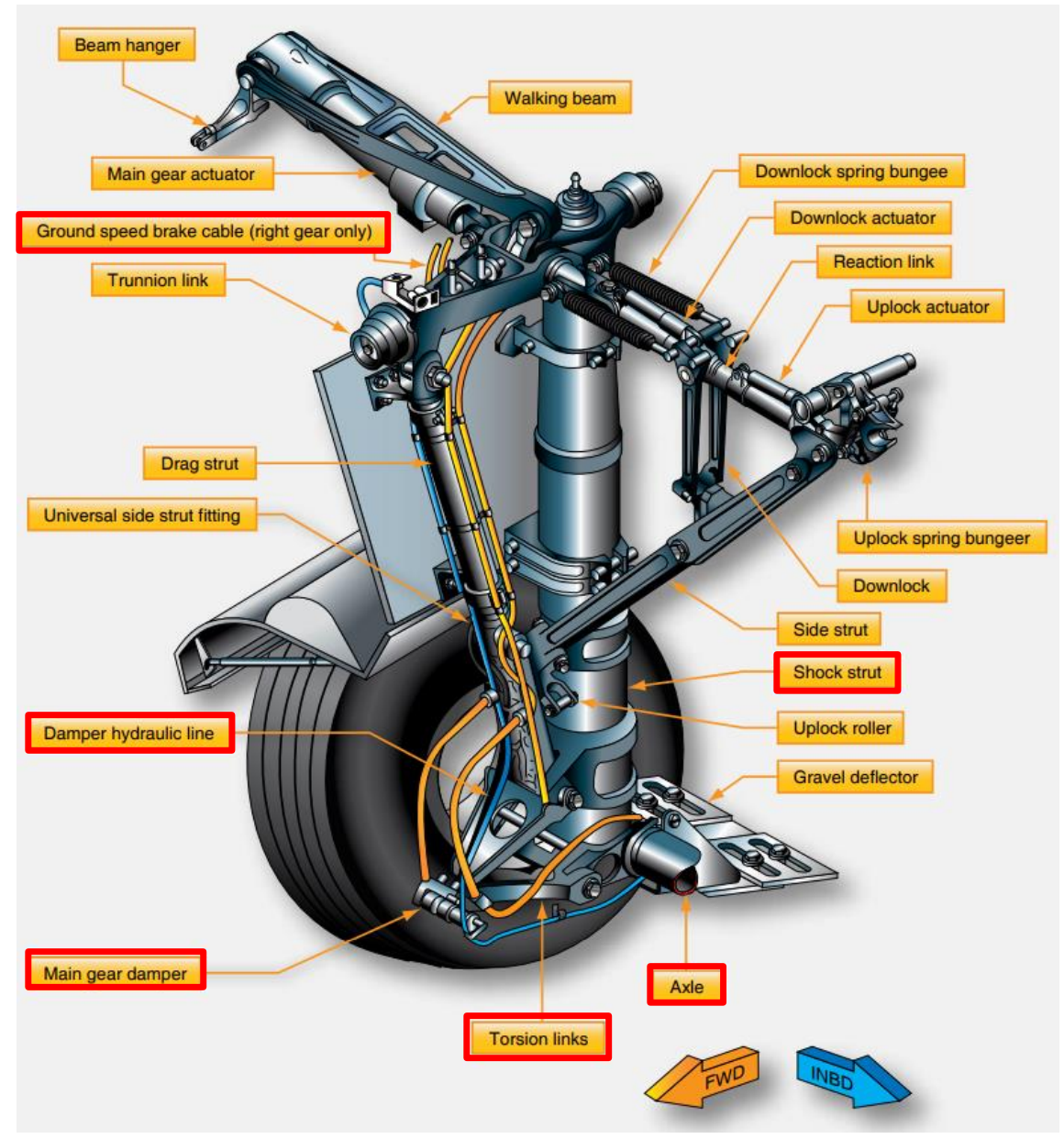

Figure 3 - List of components typically found in most commercial aircraft with a telescopic and retractable landing gear system [13]. Key components have labels outlined in red and will be referred to throughout this research.

From the diagram above, the suspension system is composed of primarily the shock strut, which house the shock absorber's cylinder and piston, the torsion link, which provides torsional stiffness and constrains the rotational motion between the shock cylinder and shock piston, the axle which connects the shock piston to the wheels, and the main gear damper, which is used to dampen 
the vibrational effects along the axis shock strut known as "Shimmy" (discussed further in Section 2.2.1). The main components that contribute to the functionality of the retraction mechanism are the main gear actuator, which drives the overall mechanism, and the trunnion link, which enables the entire gear assembly to rotate about the upper portion of the shock strut. In order to ensure the shock strut remains as rigid as possible during take-off and landing despite being connected to various components that are part of the retraction mechanism, the drag and side strut are introduced to provide structural rigidity in the longitudinal ("FWD" direction) and lateral ("INBD" direction), respectively. The downlock, uplock, and reaction link components are present to allow the side strut to remain rigid when the landing gears are deployed and flexible when retracted. Lastly, there are multiple hydraulic and electrical lines (mains and backup for redundancy) that connect from the upper portion of the shock strut to the wheel assembly.

The slave link assembly, the main focus of this research, is not shown in Figure 3 as it is a component which is specific to some select landing gear designs. As previously mentioned, the sole purpose of the slave link is to guide secondary electrical and hydraulic lines to the wheel hub. This function can be achieved through several design methodologies, as illustrated in Figure 4.
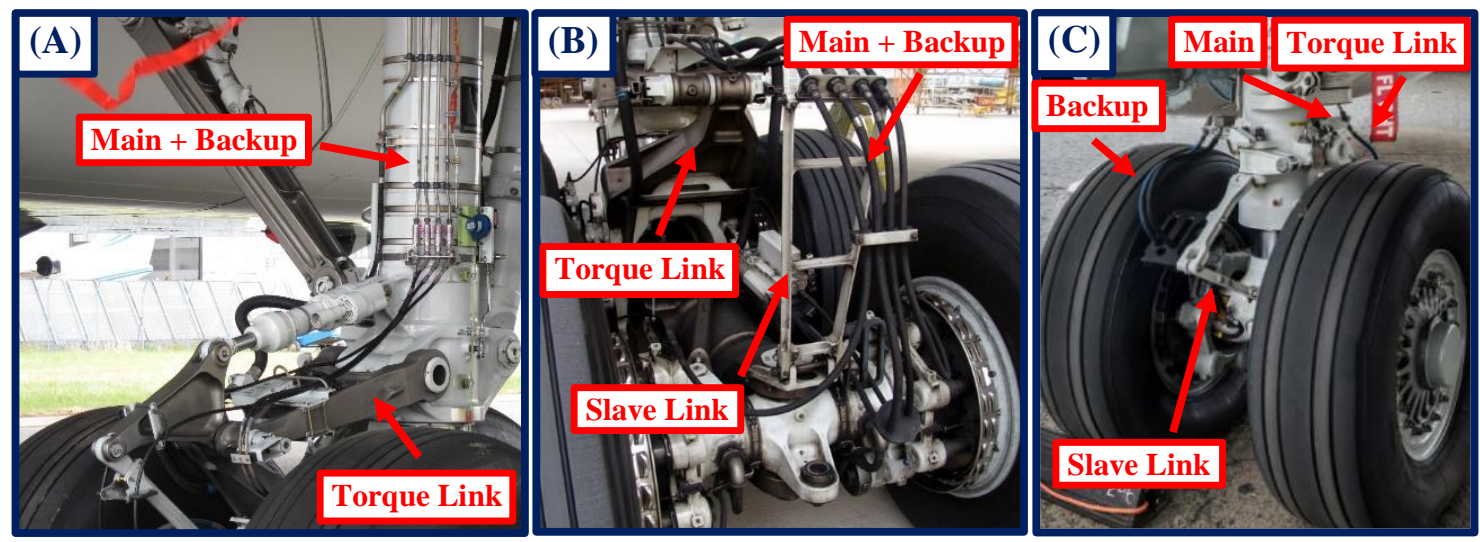

Figure 4 - (A) Boeing 747 - Main and backup lines through the torque link, (B) Boeing 777 - Both lines going through torque link and a slave link, (C) Superjet 100 - Backup lines through the slave link and main lines through the torque link. [14] [15] [16] 
A possible reason for the differences in configuration may be due to the airframe layout of the hydraulic and electrical lines. Another possible reasoning may be a difference in design philosophy, where one believes the main and backup lines should be kept physically separated to minimize the risk of simultaneous failure of both lines. Regardless of the true underlying reason behind these design decisions, key insights that should be drawn include: there are multiple ways of achieving a feasible design and there is a high degree of variances within a type of landing gear system such as the telescopic and retractable variety.

\subsubsection{Aircraft Landing Gear Configurations}

There are various configurations of landing gear layouts, but the tricycle layout is one of the most common configurations used in modern aviation, as they offer a high level of stability and maneuverability. Typically, tricycle landing gears are composed of a single nose landing gear (NLG) and two or more sets of main landing gears (MLG) located in line with the wings of an aircraft. The number of MLGs is dictated by the overall weight of an aircraft, for better load distribution when the wheels come into contact with the ground, as shown in Figure 5. Within each of these nose or main landing gear assemblies are various subassembly mechanisms that enable various functionalities, similar to the ones mentioned in Section 2.1.3.

In the unlikely event where a one-gear landing condition is unavoidable, the structural integrity and safety of the aircraft are critical. Therefore, strict policies and regulations such as CS 25.483 are set in place to ensure each landing gear are designed to withstand such extreme loading conditions [17].

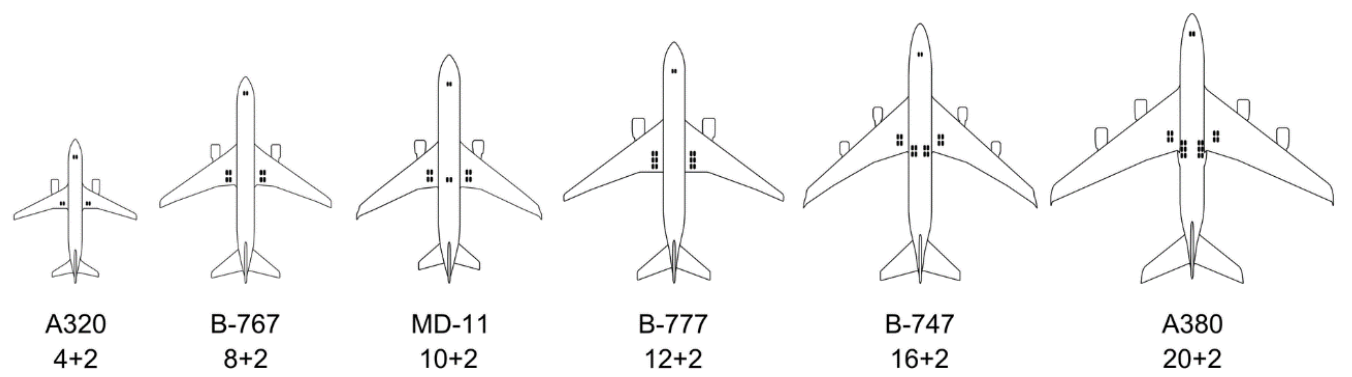

Figure 5 - Various aircraft landing gear configurations of different models. [18] 


\subsubsection{Design Considerations}

An aircraft landing gear system is a complex structure that serves various functional requirements, as described in the previous sections. The ultimate goal for the future development of this system is to continually strive to minimize the overall weight, initial and operating cost, and volume space within the airframe when retracted. Although these goals are well defined, achieving them poses many design challenges because each goal is dependent on the other. In some cases, a decrease in weight may lead to increased cost due to use of more expensive but lighter materials or additional manufacturing cost.

In order to satisfy the various strict airworthiness regulations, optimized designs must be able to meet the metrics outlined. First, the performance of the system under dynamic ground events should be analyzed and the loads experienced should be identified. Second, the system must be able to maintain structural integrity even through the worst-case loading scenarios. Third, the fatigue behavior and loading cycles for each of the components must be well defined, in order to prevent failures and establish servicing and maintenance schedules. Typically, commercial aircraft have safe component life requirements as high as 60,000 landing cycles [11]. From a more practical perspective, unconventional load cases such as accidental abuse during installation or maintenance should also be taken into account. Although these events may be difficult to quantify, it is still important to identify and consider, in order to prevent premature component failure.

Although the goals and requirements for landing gear systems have not changed significantly over time, there has been a trending decrease in development time. The growing utilization of computational tools and virtual product development is a key contributor to this shift. Verifying and validating designs with these techniques has also enabled fewer prototyping iterations before reaching a certified design, which further reduces development cost and time.

This research intends on building and expanding the capabilities of these computational tools by proposing a methodology to further improve development time through efficient and effective modeling, analysis, and optimization. 


\subsection{Landing Gear Analysis}

There are various types of analysis that go into the development of landing gear systems. For the purposes of this research, only dynamic and structural analysis will be discussed in this review. In the past, dynamic and structural analysis were typically done independently and sequentially despite being mutually dependent. Techniques for simultaneously analyzing both dynamic and structural characteristics have been developed and demonstrated in the past, however their applications to complex landing gear systems have been limited. This is likely due to the high computational cost associated with those methods that made it infeasible or impractical in the past. However, with the continual exponential growth in computational resources, these techniques may start to become feasible and will be further discussed in this section.

\subsubsection{Dynamic Analysis}

The dynamics of aircraft landing gears have been studied extensively and there are a number of notable publications. For instance, mathematical models using rigid body assumptions were developed to simulate how landing gears behave when maneuvering on the ground and validated through various experimental testing [19]. Other notable overviews in regards to aircraft ground simulation include publications by AGARD [20] and Besselink [21], which focus more heavily on accurate simulations of the shimmy phenomenon, and Krüger et al. [22], which discusses various numerical modelling approaches to simulate various ground operation scenarios, shimmy, and touch down dynamics. A number of modeling techniques proposed by Denti et al. [23] and Tadeusz et al. [24] have been utilized to accurately capture the effects of wheel ground contact and the corresponding response. A literature survey conducted by Pritchard [25] summarizes the work that has been done in the past with regards to analytical, experimental, and some computational modeling of landing gear dynamics. 
In terms of landing gear design, the two primary loading cases that are typically considered are vertical loading induced when the tire comes into contact with the ground during landing and fore/aft loading which occurs when brakes are applied to decelerate an aircraft upon landing. However, another consideration that is also of high importance in design is that there are also induced vibrational loads as a result of the aforementioned loading and can be classified by two possible phenomena: "shimmy" and "gear walk". These vibrational loads have the tendency to cause limit cycle oscillations and may cause additional mechanical wear at the joints or interface of various components that have been excited and an illustration for each phenomena is shown in Figure 6.
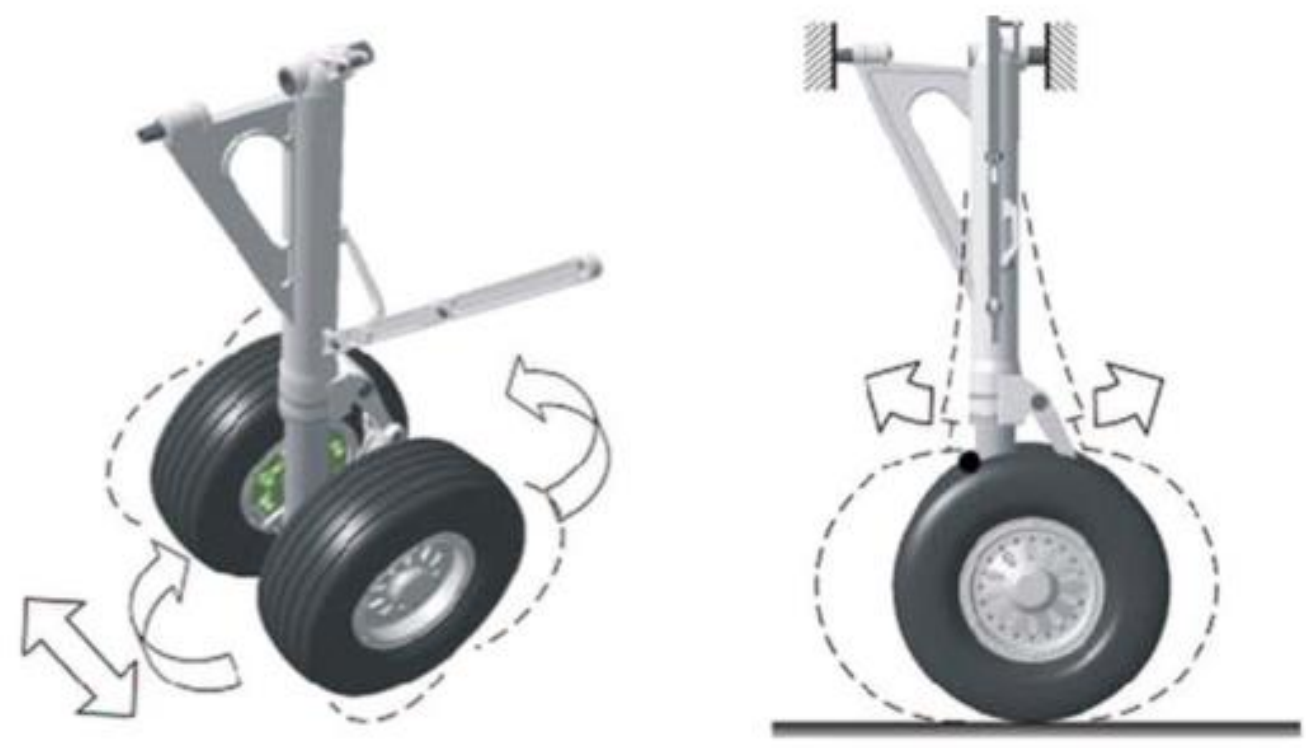

Figure 6 - Shimmy (left) and Gear Walk (right) vibrational load phenomena [26].

Shimmy is a generic term used to describe self-induced oscillations about the vertical axis of the landing gear strut. Typically, as a result of lateral bending and torsion, shimmy is considered a type of vibrational loading, which can occur in various conditions such as during taxiing, takeoff as well as landing. Similarly, gear walk is another phenomenon that is used to describe oscillations in the fore/aft direction of the landing gear, and this typically occurs during landing impact or braking. In many instances, it is possible to have both shimmy and gear walk coupled. 
The factors that contribute to these phenomena include elasticity in the landing gear components, variability in tire pressure and contact patch of different wheels, friction and free-play of pinned joints of the trunnion, side and drag strut.

\subsubsection{Structural Analysis}

In terms of structural analysis of landing gear components, the majority of the work that has been done involves simply translating loads obtained from dynamic analysis or governing equations into approximate static forces and pressure distributions. Given the narrow scope of the slave link assembly and limited work in the literature pertaining to it, three papers which analyzed various landing gear components were chosen to illustrate some of the current methodology used to perform structural analysis in the aerospace industry.

The first paper shows a nose landing gear structural analysis conducted by Nguyen et al [27]. The computer aided design (CAD) model for the cylinder, piston, fork, and wheel assembly were created using SolidWorks $@$ and the structural analysis was conducted using Algor FEA (finite element analysis). The tire was not modelled to avoid performing nonlinear analysis, which adds a substantial amount of complexity to the problem. Static pressures loads were prescribed to several components the system to model the following effects: 1.) the ground forces acting on the tire and subsequently the landing gear during landing, 2.) the reaction forces from the compression of the shock absorber and 3.) the tire pressure's influence on the wheel hub. The magnitudes of these pressure loads were derived through analytical kinematics and Federal Aviation Administration (FAA) approximation standards. The fixed boundary condition was applied to the two bolt connections to which the landing gear mounts onto the bulkhead and contact surfaces were generated to model the interaction of multiple components. Displacement and stress contours were shown, along with a summary table for the factor of safety of each component.

The second paper shows a similar analysis done by Gowda [28], who also conducted a structural analysis on another nose landing gear design as well as a subsequent stress-life fatigue 
analysis. For this paper, CATIA@ was used as the CAD modeling software, MSC Nastran $\odot$ was utilized to conduct the structural analysis, and the fatigue analysis was conducted with stress-life equations in Microsoft Excel@ $\odot$. The loads prescribed to the system were in the form of point forces applied to the two ends of the axle in the three principal directions. Multiple load cases were generated by varying the magnitude of the loads in each direction, replicating the various ground maneuvering events such as taxing, turning, and towing to name a few. Again, fixed boundary conditions were applied to the connection points of the airframe, however rigid elements were used to model the connection between the various landing gear components. A possible justification for this may be due to the high number of components in the model analyzed by Gowda, relative to the model analyzed by Nguyen. Stress and displacement results were generated and later used to obtain damage results from fatigue analysis.

The third paper demonstrates an in-depth analysis and prototyping of a dual locking linkage mechanism that was carefully conducted by Oh [29]. CATIA@ was again used as the CAD modeling software. A key difference to the methodology used by Oh that separates his work from that of the previous two is the utilization of computational tools to obtain loads specific to the design. Kinematic and dynamic analysis was performed in ADAMS $\odot$ for the entire landing gear assembly and the loads experienced by the link were obtained. These loads were then used in a subsequent static structural analysis to obtain the stress and displacement contours for the linkage mechanism under tensile and compressive loading.

Based on this brief overview of the methodology used to conduct structural analysis for landing gear systems, one can begin the notice a high degree of variability in terms of where the loads are obtained. The use of standards and analytical techniques may give a good first approximation to the magnitudes of the actual loads experienced. Although it is accepted in the aerospace industry to utilize this method with conservative estimates, this may lead to overdesigned and non-optimized components. Performing dynamic analysis on specific landing gear systems is essential to obtain accurate load magnitudes, as shown in the work done by Oh. However, this 
method would likely add a substantial amount time to the development cycle, as two separate analysis, dynamic and structural, would need to be conducted. While this is necessary, the application of this process may be limited to only critical components due to the inefficiencies of this approach.

\subsubsection{Multi-Body Dynamic Analysis}

Although the dynamics of each mechanism independently is well understood, the coupling and interactions between multiple mechanisms creates complexities in modeling and analysis. To add to these complexities, structural performance is often a crucial aspect to consider in the design of landing gears in order to ensure components are able to withstand various time-dependent loading conditions. The coupling of dynamic and structural analysis is inherently a difficult problem to solve due to the number of nonlinearities introduced as well as the increased computational cost involved with analyzing multiple components. Despite these challenges, recent improvements and implementation of multi-body dynamic (MBD) solvers into commercial FEA software suites have enabled the potential for dynamic and structural aspects to be considered within a single analysis.

From this, the MBD analysis of various landing gear mechanisms was another important area of research to review. Initial searches yielded examples that focused more on the dynamic aspects of the analysis. For example, a single-sidestay mechanism was successfully modelled using numerical continuation analysis and a rigid-body assumption in an article published by Knowles [30]. However, vibrational loading is a highly important loading scenario to consider in landing gear design. It is desirable to model components as deformable bodies, rather than rigid bodies, because the behavior of these oscillations are affected by the elasticity of the landing gear frame. This has been shown to be the case in another article published by Krüger et al. [26], which discussed the differences in the stability simulated responses for a various landing gear components 
modeled with rigid and flexible components under oscillating phenomenon such as shimmy and gear walk.

Improvements in computing power and methods have given rise to techniques that now exist to couple MBD and structural analysis through flexible elements. Techniques for deformable MBD modeling and simulation outlined in publications by Shabana [31], Bauchau [32], and Cardona [33] have been implemented in modern FEA software packages with practical example results from Xingguo el at. [34]. Although these concepts have existed for some time, no work has been performed for landing gear slave link systems.

\subsection{Design Optimization}

Design optimization can be considered to be the process of determining the best set of parameters to yield the greatest performance gain, while satisfying a particular set of rules or constraints. The process of determining the best set of parameters is typically done iteratively, through either heuristic or systematic approaches. For the systematic approach, a design problem may be mathematically formulated in the following general form [35]:

minimize $f(\mathbf{X})$

subject to $\begin{cases}h_{i}(\mathbf{X})=0, & i=1,2, \ldots, m \\ g_{j}(\mathbf{X}) \leq 0, & j=1,2, \ldots, r \\ \mathbf{X} \in S . & \end{cases}$

where $f(\mathbf{X})$ is the objective function, $h_{i}(\mathbf{X})$ and $g_{j}(\mathbf{X})$ are the equality and inequality constraints, respectively, $\mathbf{X}$ is a vector of design variables, $S$ is a subset of the feasible design space, and lastly $m$ and $r$ are the number of equality and inequality constraints, respectively. From the optimization statement shown, it can be seen that problem is setup in a manner where the objective and constraints are a function of the design variables. Therefore, a systematic updating scheme for the design variable may be implemented to drive the objective function towards an optimal solution, while ensuring the defined constraints are satisfied. There are various approaches to implementing 
said updating scheme, however gradient-based methods [35] including the method of moving asymptotes (MMA) [36], genetic algorithms [37], and evolutionary algorithms [38] are some of the popular methods used and researched in the aerospace industry.

For the purposes of this research, two types of design optimization will be studied and utilized in the proposed methodology.

\subsubsection{Topology Optimization}

Topology optimization is a type of design optimization defined by the process of determining the optimal design layout of material and connectivity inside a user-specified design domain and constraints. An example is shown in Figure 7.
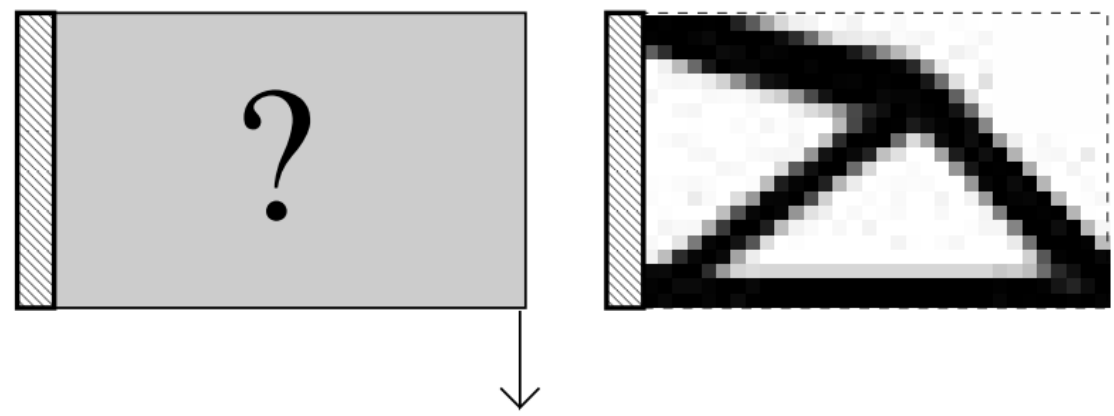

Figure 7 - 2D Topology Optimization Example. Design domain, fixed boundary, and point load is defined (left) and the resulting optimized structure (right) [39].

There are various formulations for the topology optimization problem statement, however the most generic form is as follow [40]:

minimize $\quad C\left(\rho_{i}\right)=\mathrm{U}^{\mathrm{T}} * \mathrm{~K} * \mathrm{U}$

subject to $\left\{\begin{array}{l}K\left(\rho_{i}\right) * U=f \\ \sum_{i=1}^{n} \rho_{i} v_{i}-V^{*} \leq 0 \\ 0 \leq \rho_{i} \leq 1, \quad i=1,2, \ldots, n\end{array}\right.$

where $C\left(\rho_{i}\right)$ is compliance of the design domain, $K\left(\rho_{i}\right)$ is the global stiffness matrix, $U$ is the displacement vector, $f$ is the static load vector, $\rho_{i}$ and $v_{i}$ are the density and volume of the $i$-th element, respectively, $V^{*}$ is the volume fraction constraint value, and $n$ is the total number of design 
variables. Compliance is typically chosen as the objective function, because it is numerically stable and the sensitivities are well defined. Common values for the volume fraction constraint ranges from 0.2 to 0.4 , and set based on the volume of the design domain. This is done to ensure the target volume is set to a value that lower than the volume of the baseline design.

Topology optimization has become an effective design tool in the aerospace industry for achieving significant weight savings and performance improvements [8]. By efficiently utilizing the available design freedom allocated, optimized designs produced from this approach consistently outperform ones generated from other optimization techniques such as shape and size. Examples for successful applications of topology optimization in the aerospace industry include the Airbus A380 leading edge ribs [41], Airbus A320 nacelle hinge bracket [42], and Airbus A350 turbine engine pylon [43].

Typically done with linear static load cases, topology optimization is not readily applicable for landing gear systems because of the complexities that come from dynamic loading and interaction between various components. Approximations for the magnitudes of the static loads may be made, although this has the potential to lead to suboptimal results as the load cases used to describe the behavior of the system may not be accurate. The alternative is to conduct a fully nonlinear topology optimization, however this is an undesirable technique to use because it is computationally very expensive to the point where it is not practical. Topology optimization with geometric and material non-linearity has been done with some success but only for simple test case geometries [44].

\subsubsection{Multi-disciplinary Optimization}

Multi-disciplinary Optimization is defined by the use of optimization methods to solve a design problem involving a number of different disciplines. The design optimization of the slave link assembly falls into this category, as it is important to consider both dynamic and structural aspects in the topology optimization process. 
A method that shows great promise in resolving these issues and enabling multidisciplinary topology optimization is the use of the Equivalent Static Load Method (ESLM). The methodology behind ESLM has been described in the works of Choi et al. [45] and Park et al. [46]. By taking the displacement field of each time step from a particular non-linear analysis and transforming them into multiple equivalent static load cases, the ESLM has the capability of enabling optimization with various non-linear analysis as an input or objective function. In the case of MBD analysis, this is achieved by systematically taking the compliance of the system at various time steps and converting them into linear-static load cases for topology optimization. A diagram summarizing this process is shown in Figure 8.

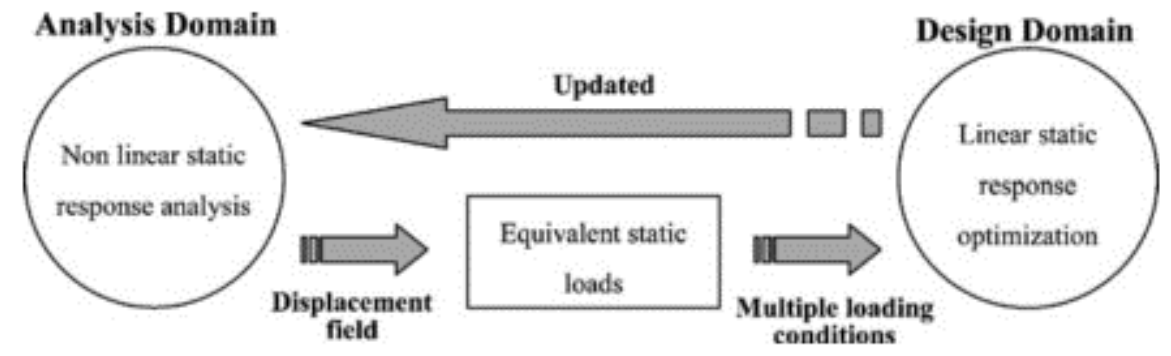

\section{Figure 8 - Diagram outlining the ESLM process.[46]}

The ESLM has been proven successful in various simple test case problems and even some practical problems in other industries. The early works of Kang et al. demonstrated the use of the ESLM to optimize 2D-truss structures [47]. More recently, similar works were done by Li et al., however 2D-voxel elements were used instead of trusses [48]. Sun et al. presented one of the first examples for the use of optimization with ESLM for 3D structures, where components of a slidecrank mechanism were successfully optimized with this method [49]. Other than a white paper published by Altair Engineering on the potential to utilize this technique for landing gear systems without concrete methodologies and results [9], there has been no mention found of any aerospace components that considered MBD analysis and topology optimization with ESLM. 


\subsection{Literature Review Overview}

In summary, the literature review has provided a high-level overview of landing gear design, analysis, and optimization. To reiterate a key recurring theme in the aerospace industry, there is a growing demand to produce more lightweight and cost efficient components with less development time and prototyping iterations. It is therefore important to continue to explore new approaches, in order to generate designs for real world applications in an accurate yet efficient manner. The multi-disciplinary topology optimization with ESLM to consider both dynamic and structural aspects of a landing gear assembly has yet to be performed and documented with a detailed methodology. This research could prove to be a highly beneficial design tool for the aerospace industry. 


\subsection{Chapter 3: Design Optimization Process}

The focus of this research was to perform high-fidelity multi-disciplinary design optimization to reduce the weight and cost of a slave link assembly while simultaneously satisfying structural and dynamic performance requirements. The goal of the optimization was to develop multiple designs to introduce either $30 \%$ weight savings, $20 \%$ cost savings, or both simultaneously. Detailed drawings for the slave link assembly was provided by Safran Landing Systems to conduct this research and CAD models were generated using SolidWorks $@$. Altair Hyperworks@ software suite was used to create finite element model as well as perform subsequent MBD analysis and topology optimization with Altair's Optistruct $\odot$ solver.

\subsection{Problem Definition}

For this research, two components were selected as the main candidates to conduct design optimization, the upper slave link (USL) and lower slave link (LSL) for the MLG of a Sukhoi Superjet-100 aircraft. Figure 9 shows the physical baseline design installed on an aircraft in operation, with the USL and LSL highlighted.

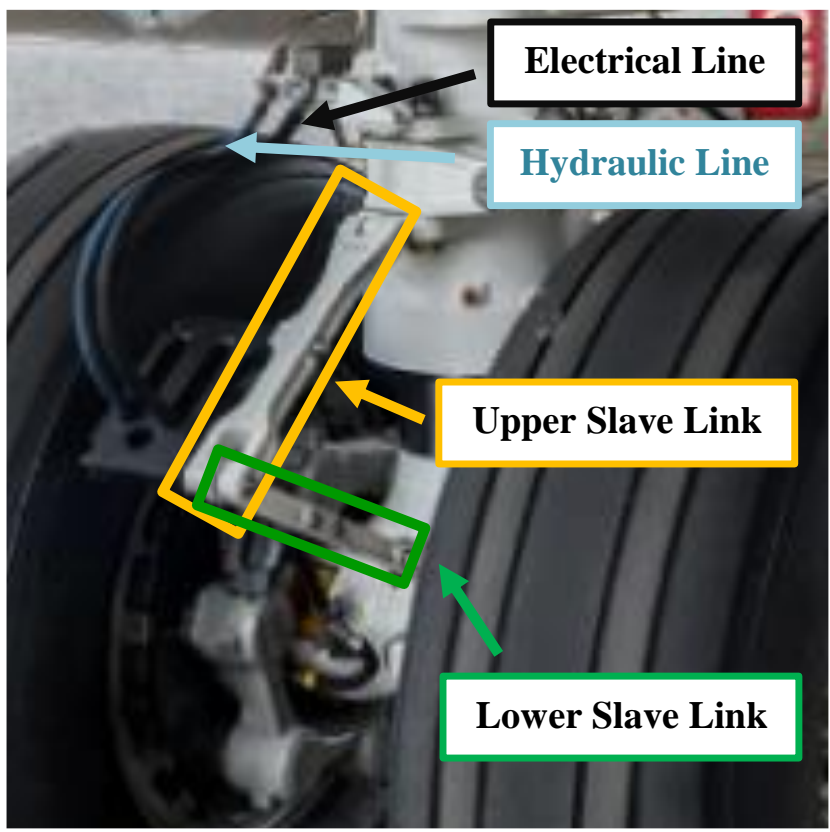

Figure 9 - Slave link assembly for a Sukhoi Superjet 100 aircraft. [16] 


\subsection{Design Requirements and Constraints}

To reiterate, the sole purpose of the slave link assembly is to guide secondary hydraulic (blue) and electrical (black) lines from the upper portion landing gear to the wheel hub. The slave link components are considered tertiary components, and therefore do not require expensive and time-consuming recertification testing to be put into service. As long as revised designs generated from this research can be justified to be a design improvement computationally, subsequent experimental testing may conducted to ensure the designs meet the internal requirements of Safran for implementation.

Through continuous communication and collaboration with Safran Landing Systems, the following design requirements and constraints were defined and implemented in the optimization process. It should be noted that any requirement and constraint not explicitly listed below may be assumed to be a design freedom.

1. Loading Conditions - Subject to interpretation, the revised designs must be able to withstand the worst possible landing scenarios. An example of a static-equivalent loading typically used for this scenario were given as a

a. 20g (gravity) load applied in the forward-aft direction for each component

b. 20g load applied in the lateral direction for each component

2. Component Mounting - The following connection points may not be changed, as they affect the geometry of other components which are outside the scope of this research:

a. Pin connection between the upper slave link and the cylinder of the shock strut

b. Pin connection between the lower slave link and the wheel hub

3. Manufacturing - In order to fully utilize the manufacturing capabilities available at Safran and to help ensure cost savings are met, it was recommended that the revised designs be manufacturable through the means of computer numerical control (CNC) machining such as milling. 
4. Material Selection - Although there were no explicit restrictions against the use of alternative materials, it was strongly advised to keep the material selection the same as the original design because it would simplify post-processes such as anodizing and corrosion protection. For this reason, 7000-series aluminum was selected as the material choice for the revised design.

\subsection{Process overview}

To approach the design optimization in a systematic manner, the proposed methodology implemented in this research was partitioned into three design stages. These stages are defined as Stage 1 - Setup, Stage 2 - Optimization, and Stage 3 - Redesign. There are multiple aspects and sub-processes considered within each stage, outlined in Figure 10. The subsequent chapters will be based around each stage and a high level overview is provided below.

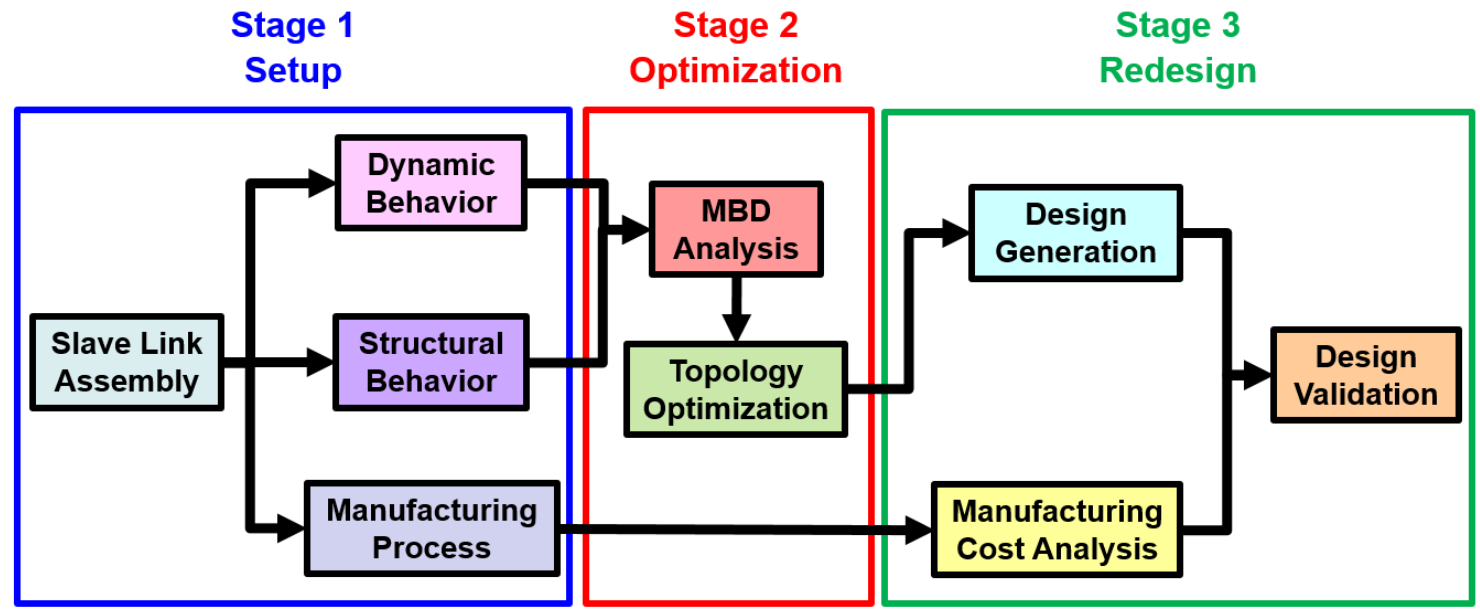

Figure 10 - Slave link assembly design optimization process overview

The first stage of this research covers the creation and setup of the models for analysis and optimization. Detailed drawings for the slave link assembly components were modelled in CAD. In order to properly set up the model for MBD analysis and subsequently topology optimization without introducing unnecessary complexities, a simplified landing gear test rig model was created 
for the attachment of the slave link assembly. This was done to closely replicate the behavior of the physical system. The finished CAD assembly was then exported into Altair's Hypermesh to create the finite element model. Next, the dynamic behavior for the shock absorber compression, shimmy and gear walk vibrational loading was quantitatively defined through mathematical equations and experimental results. The structural behavior characterization involved identifying the material properties, body definitions, and joint definitions for each component in the model. Definitions and methodology for the manufacturing process of the slave link components were selected in this stage, while the analysis was conducted at a later stage, once the optimization results were produced.

In stage two, the finite element model created in the previous stage was utilized to conduct MBD analysis. The results for the dynamic and structural responses of the system were first examined, to ensure the results accurately reflected the behavior of the physical system. Once verified, the finite element model of the landing gear was reconfigured to accept design spaces for the two slave link components to be optimized. Multiple topology optimization runs were then performed with various optimization parameters and active constraints. Topology isosurfaces for the optimized structures were generated, along with convergence plots to observe the behavior of each optimization run.

Finally, stage three involved producing revised designs from the results of the topology optimization. Isosurfaces were exported into the CAD program for design reinterpretation. Manufacturing process definitions from the first stage were utilized in this stage to perform a manufacturing cost analysis, estimating the relative change in cost compared to the baseline design. Two approaches were taken in the design generation process, where one focused solely on maximizing the weight savings, and the other on meeting both weight and cost saving targets. Once created and evaluated, these revised designs were then reconfigured into the landing gear finite element model, to validate the dynamic (displacements, and accelerations) and structural (stresses and strains) performance of the new components. 


\subsection{Chapter 4: Computational Model Setup}

The purpose of this stage of the research was to produce the basis and foundation of which the proposed methodology would build upon. One of the key benefits of this methodology was attempting to achieve was an efficient and effective computational model with which the baseline design could be analyzed and later reconfigured to accept revised designs for a comparative analysis. In order to accomplish this, the model had to be versatile and practical. The next step was to define and identify the dynamic and structural behavior for the worst-case loading scenario. Experimental testing data and practical experience were heavily utilized to obtain these behaviors and later implemented into the model. The manufacturing process for the slave link components was $\mathrm{CNC}$ milling; to predict the cost savings associated with the design revisions, a model was created based on various parameters such as the cost of each operation and stock material volume. The details for each aspect of the setup will elaborated in the sections below.

\subsection{CAD Modeling and Assembly}

Detailed drawings for the upper slave link and lower slave link were provided by Safran Landing Systems and a CAD model was recreated using SolidWorks@. In order to establish an accurate baseline, these models were created with the exact dimensions and features specified. Drawings and CAD models for the upper slave link and lower slave link are shown below in Figure 11 and Figure 12, respectively. All dimensions listed are in $\mathrm{mm}$. Due to confidentiality, only the overall dimensions of each component are shown.
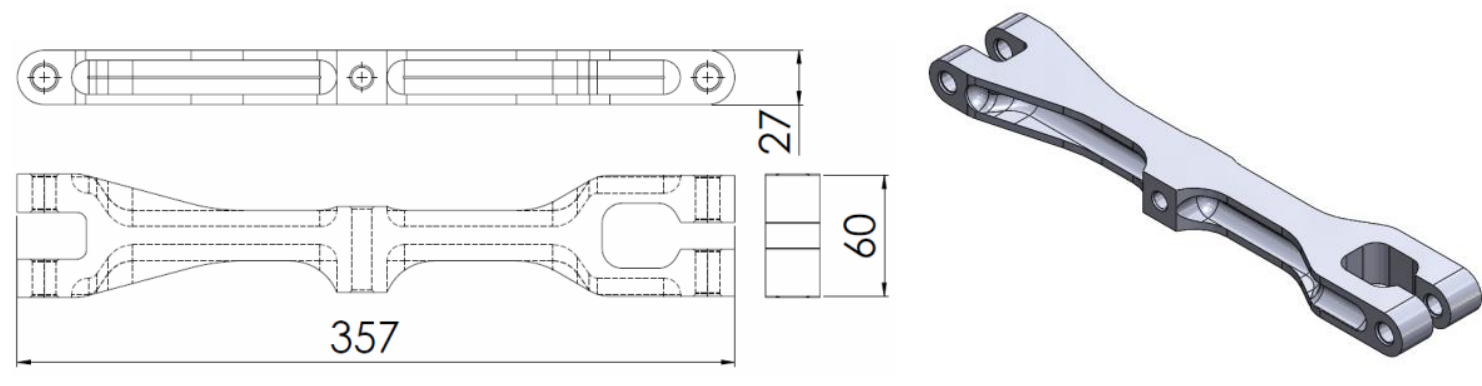

Figure 11 - Simplified upper slave link drawing (left) and CAD model (right). 

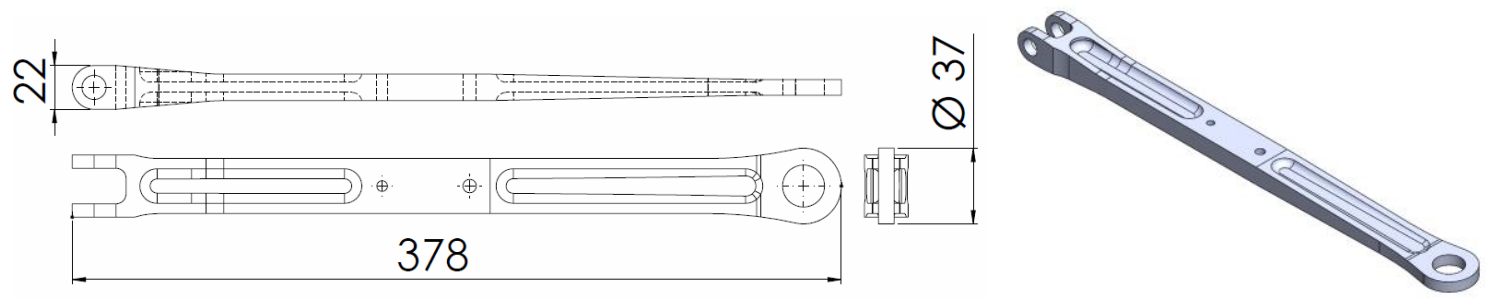

Figure 12 - Simpified lower slave link drawing (left) and CAD model (right).

The slave links are manufactured from 7000-series aluminum. As previously mentioned, the primary purpose of these two linkage components are to act as cable guides for secondary hydraulic and electrical lines. Attached to the linkage components are Delrin cable guides, which come into direct contact with the lines, reducing sliding friction when the landing gear is in motion. Since the detailed drawing files for these components were not provided, a simplified and approximated version of the upper (Figure 13) and lower (Figure 14) Delrin cable guides were created from an assembly view of the landing gear to simulate the inertial effects it has on the slave link components.
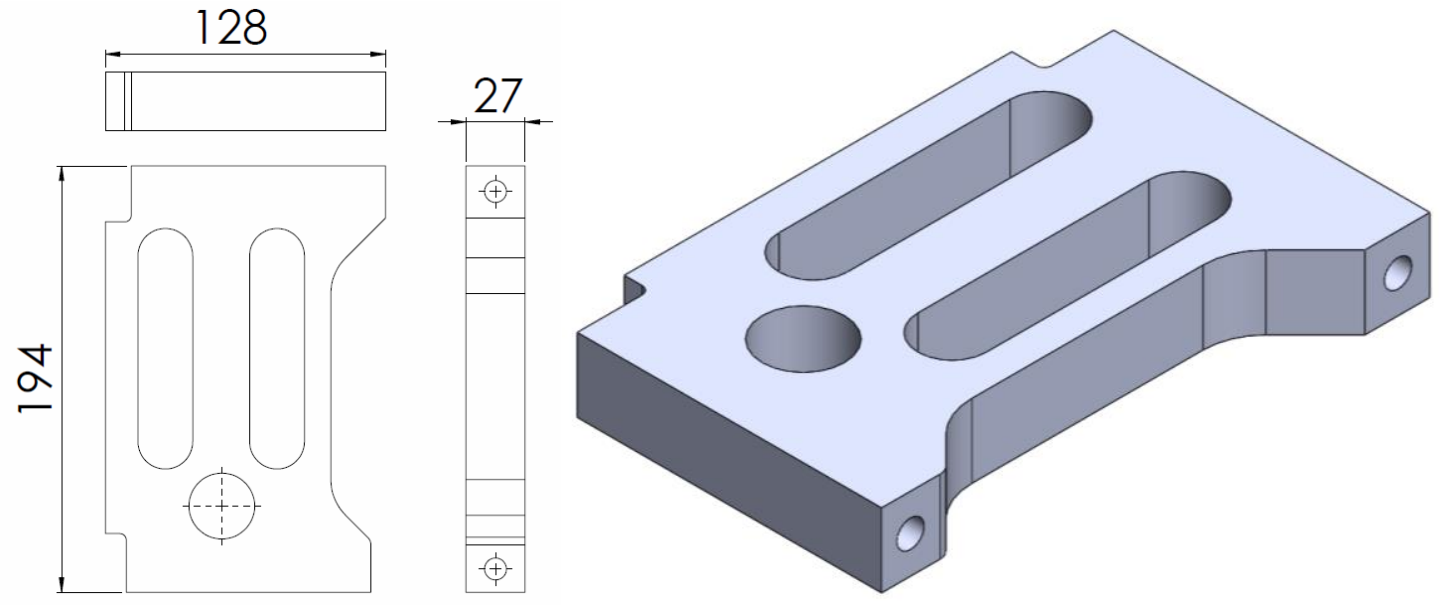

Figure 13 - Simplified upper cable guide drawing (left) and CAD model (right). 


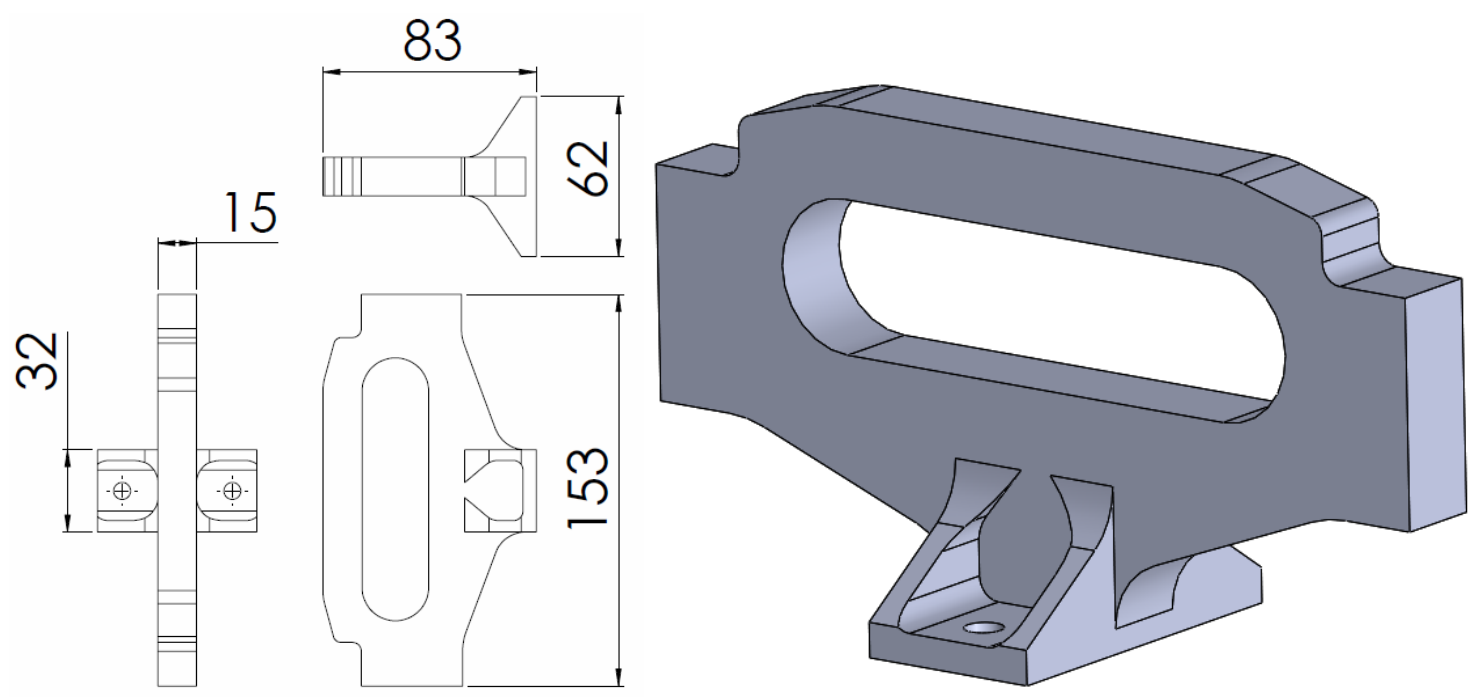

Figure 14 - Simplified lower cable guide drawing (left) and CAD model (right).

The upper slave link was connected to the upper strut through a revolute joint, a ball joint between the upper and lower slave link, and universal joint to connect the lower slave link to the lower strut. This joint configuration was selected to allow the mechanism to have the necessary degrees of freedom to accommodate for the vibrational loading present. The material used to model the pin connections was stainless steel. The pins were not fully modelled for reasons that will be further discussed in the finite element modeling section of this research (Section 4.2).

To save on computational modeling resources, the upper and lower strut CAD geometries were simplified significantly, as they were not the main focus of this research. To ensure the slave link assembly would behave in a similar manner with the physical system, the location for the upper and lower strut connection point were kept consistent. Additionally, the travel distance of the lower strut was modelled to closely match the one seen in the assembly drawing provided. The entire CAD assembly is shown in Figure 15. Note that the secondary lines were modeled for visualization purposes only and were not included in the subsequent analysis. The justification for this is based on the assumption that the secondary lines have negligible structural effects to the slave link assembly. 


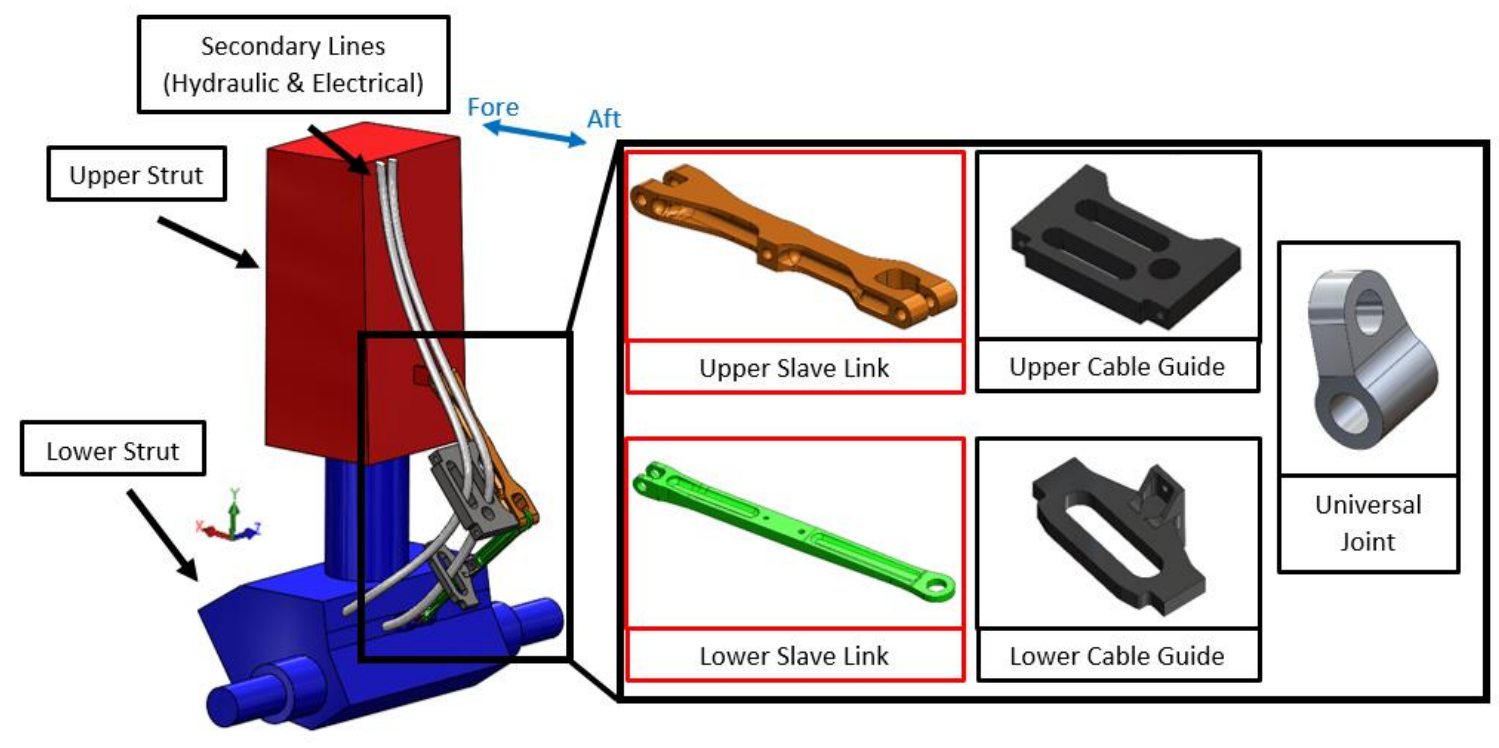

Figure 15 - Slave link assembly components on a simplified landing gear CAD Model. Design optimization was applied to the upper slave link and lower slave link.

\subsection{Finite Element Modelling}

A finite element model was created from the geometries in the CAD assembly model in Altair's Hypermesh software. The entire meshed model of the slave link assembly and landing gear test rig can be found in Figure 16 and was composed of approximately 535,000 finite elements and 861,000 nodes. The mesh density was determined from preliminary mesh sensitivity analysis. The coordinate system will remain constant for the entirety of this research and references to axis directions will be based on Figure 16. The top grey block acts as a grounding point for the multibody dynamic model and represents the point of connection for the landing gear to the aircraft frame. Unit consistency was an important aspect to maintain throughout the model setup process to ensure precision in the analysis. Table 1 summarizes the units used in the development of the model.

Table 1 - Unit Consistency for the slave link landing gear model

\begin{tabular}{ccccccccc}
\hline Mass & Length & Time & Force & Pressure & Energy & Velocity & Density & Gravity \\
\hline $\mathrm{Mg}$ (ton) & $\mathrm{mm}$ & $\mathrm{s}$ & $\mathrm{N}$ & $\mathrm{MPa}$ & $\mathrm{mJ}$ & $\mathrm{mm} / \mathrm{s}$ & $\mathrm{Mg} / \mathrm{mm}^{3}$ & $9.81 \mathrm{E}+03 \mathrm{~mm} / \mathrm{s}^{2}$ \\
\hline
\end{tabular}



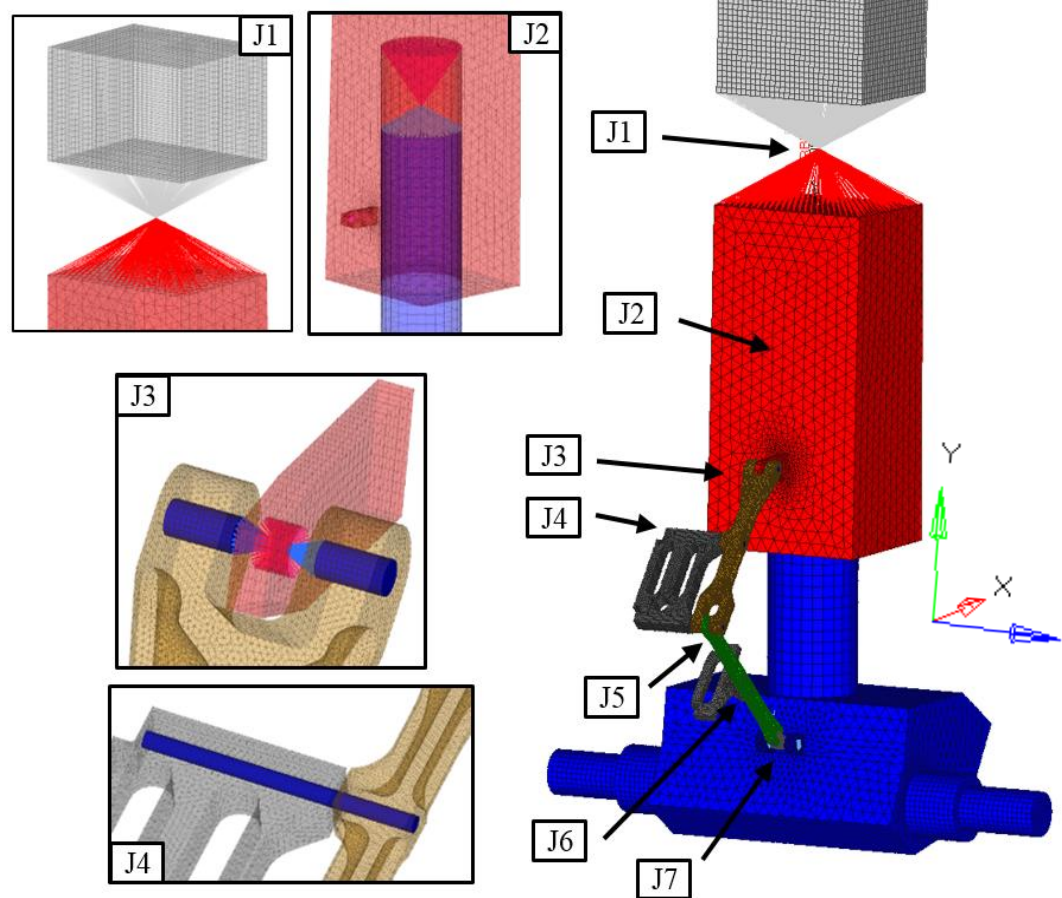

\section{Ground Component}
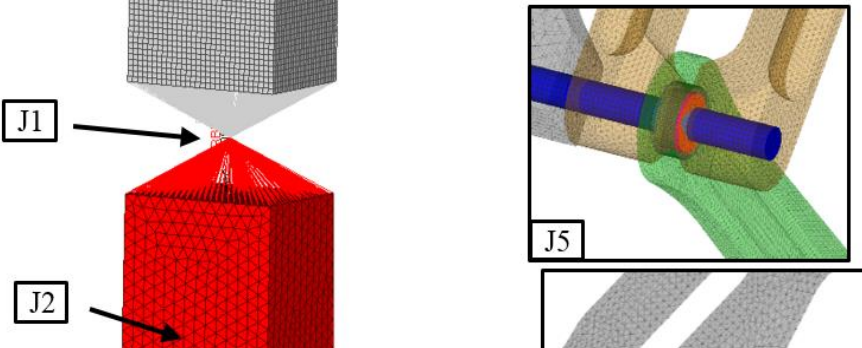

Figure 16 - Finite element model of slave link assembly on simplified landing gear test rig.

In order to define joints with specific degrees of freedom (DOF) enabled to replicate the correct motion, two coincident nodes, one from each component being attached, must be created and linked. A fully unconstrained node has six DOF, which are comprised of three translational (TRANS) DOF and three rotational (ROT) DOF, each corresponding to a principal axis X, Y, and Z. The joints defined in Altair OptiStruct $(\mathrm{C}$ and utilized in the landing gear model are shown in Figure 17.

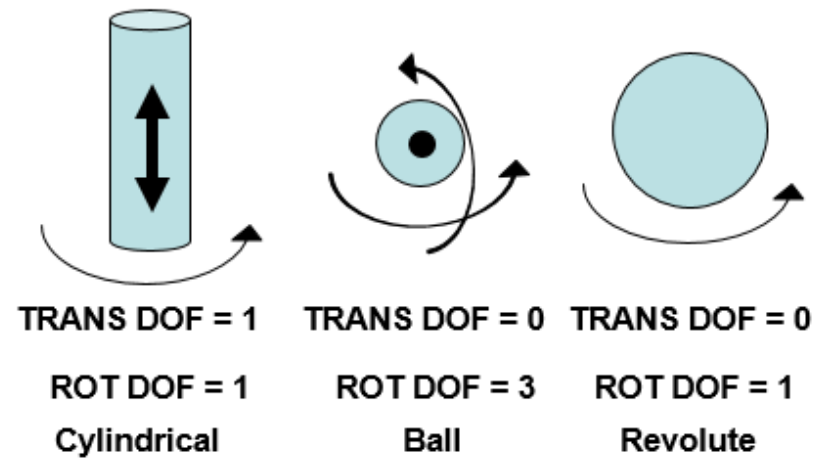

Figure 17 - Joint definitions available in Altair Hyperworks and utilized in the model. 
There are various techniques to attach joint nodes to components with different advantages and disadvantages. The simplest and most computationally efficient technique for joining was to use rigid (RBE2) elements between the joint node (independent) and the nodes on the inner faces of the hole to which a pin would slot in (Figure 18-A). The disadvantage to this technique was that it over-stiffened the joint and led to inaccuracies in the subsequent results. An alternative technique was to use solid elements within the pinholes and connect the rigid elements to the face of the solid elements. The solid elements could then be given material properties to match that of the physical pins and allows for small deformations of the pin to be captured by the model (Figure 18-B). The disadvantages were that this approach was more computationally costly than just using rigid elements, and there were still some inaccuracies present. For instance, when the pin was under bending, one side of the pin experienced compression loading due to contact with the component. As a result of the solid element mesh being connected with the component elements, however, the opposite side of the pin experienced an artificial tension loading equal to the compression load. A technique to prevent this from occurring was to introduce contact elements between the solid pin elements and the corresponding mating hole (Figure 18-C). This would have improved the accuracy of the result; however, the computational cost for running the model would have also increased. With the number of different joints present in the model, contact elements had the potential to introduce unnecessary numerical instabilities. 

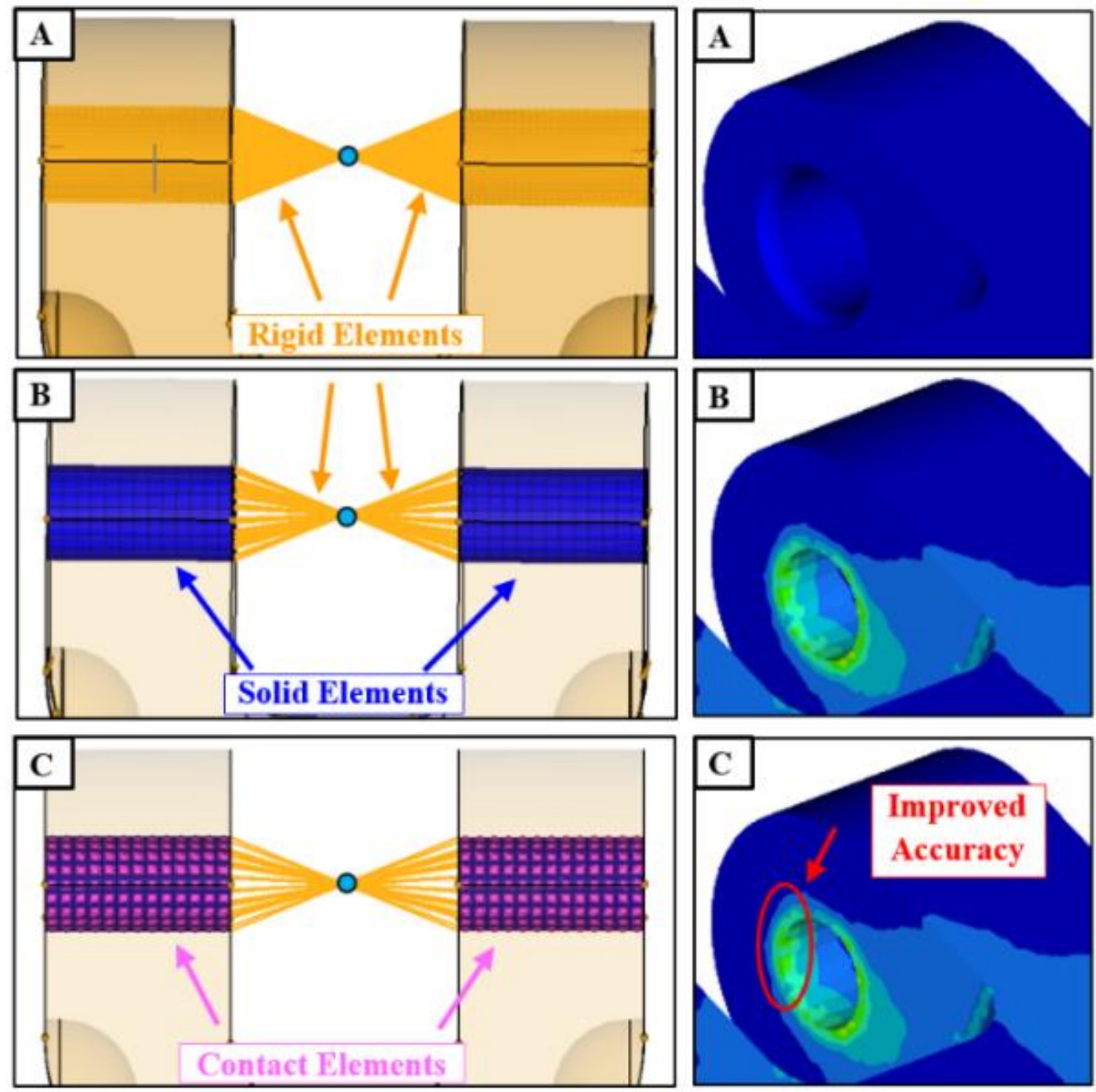

Figure 18 - Joint modeling techniques and effects on analysis.

Since the end purpose of this model was to conduct topology optimization, solid pin elements (Figure 18-B) were utilized as the technique for connecting joint nodes to components. They were deemed to provide sufficient levels of accuracy without adding a substantial amount of computational cost. The trade-off with the slight loss in accuracy for better computational performance was further justified by the additional decrease in user setup time. Although this may not be significant for a single analysis, the benefits are quickly realized for analyses that require multiple iterations or reconfiguration. 
Once the joints were modelled with solid pin elements, joint definitions were prescribed to each joint in a manner which matched the physical system. To enable the landing test rig to experience gear walk, a revolute joint (J1) was imposed between the upper strut and the fixed ground block to simulate play in the trunnion joint and allow for a pivot motion about the Z-axis. Similarly, a cylindrical joint (J2) was given to the connection between the upper and lower strut to provide the necessary degrees of freedom (D.O.F.) for the shock absorber compression and shimmy motion. Based on the drawings of the physical system, the following joint definitions were prescribed: a revolute joint (J3) between the upper strut and upper slave link, a ball joint (J5) between the upper slave link and lower slave link, and two revolute joints (J7) to replicate a universal joint where the two axis of rotations are offset from one another to connect the lower slave link to the lower strut. The connection between the cable guide and slave link for both upper (J4) and lower (J6) were modelled using solid pin elements with no D.O.F. The joint definitions are summarized in Table 2.

Table 2 - Component Joint Definitions

\begin{tabular}{llllll}
\hline ID & Component 1 & Component 2 & Joint Type & $\begin{array}{l}\text { Rotational } \\
\text { DOF }\end{array}$ & $\begin{array}{l}\text { Translational } \\
\text { DOF }\end{array}$ \\
\hline J1 & Ground (Fixed) & Upper Strut & Revolute & 1 & 0 \\
J2 & Upper Strut & Lower Strut & Cylindrical & 1 & 1 \\
J3 & Upper Strut & Upper Slave Link & Revolute & 1 & 0 \\
J4 & Upper Cable Guide & Upper Slave Link & Solid Pin & 0 & 0 \\
J5 & Upper Slave Link & Lower Slave Link & Ball & 3 & 0 \\
J6 & Lower Cable Guide & Lower Slave Link & Solid Pin & 0 & 0 \\
J7 & Universal Joint & Lower Slave Link & Revolute & 1 & 0 \\
J7 & Universal Joint & Lower Strut & Revolute & 1 & 0 \\
\hline
\end{tabular}




\subsection{Dynamic Behavior Characterization}

Before conducting an MBD analysis, various types of motion experienced by a landing gear were carefully studied and characterized. The findings from the literature review led to the definition of three input motions that would lead to replicating the behavior of the physical system. The first motion involves the translational motion of the lower strut because of the shock absorber compressing upon landing (Figure 19). The second and third motion involve vibrational loads induced on the system during landing such as shimmy and gear walk. To replicate these phenomena, a rotational motion about the $\mathrm{Y}$-axis was prescribed to the lower strut in place of shimmy (Figure 20) and a rotational motion about the Z-axis is prescribed between the upper strut and the ground block (Figure 21).

The translational motion $u(t)$ of the lower strut can be described mathematically with the following second order response equation:

$$
\begin{aligned}
& u(t)=A_{1} *\left[1-\frac{1}{\beta} e^{-\zeta_{1}\left(2 \pi f_{1}\right) t} \sin \left(2 \pi f_{1} \beta t+\alpha\right)\right] \\
& \beta=\sqrt{1-\zeta_{1}^{2}} \\
& \alpha=\cos ^{-1} \zeta_{1}
\end{aligned}
$$

where $\mathrm{t}$ denotes the time-domain, $A_{1}$ represents the final compressed position of the lower strut, $f_{1}$ is the frequency at which the response occurs, $\zeta_{1}$ is the damping ratio.

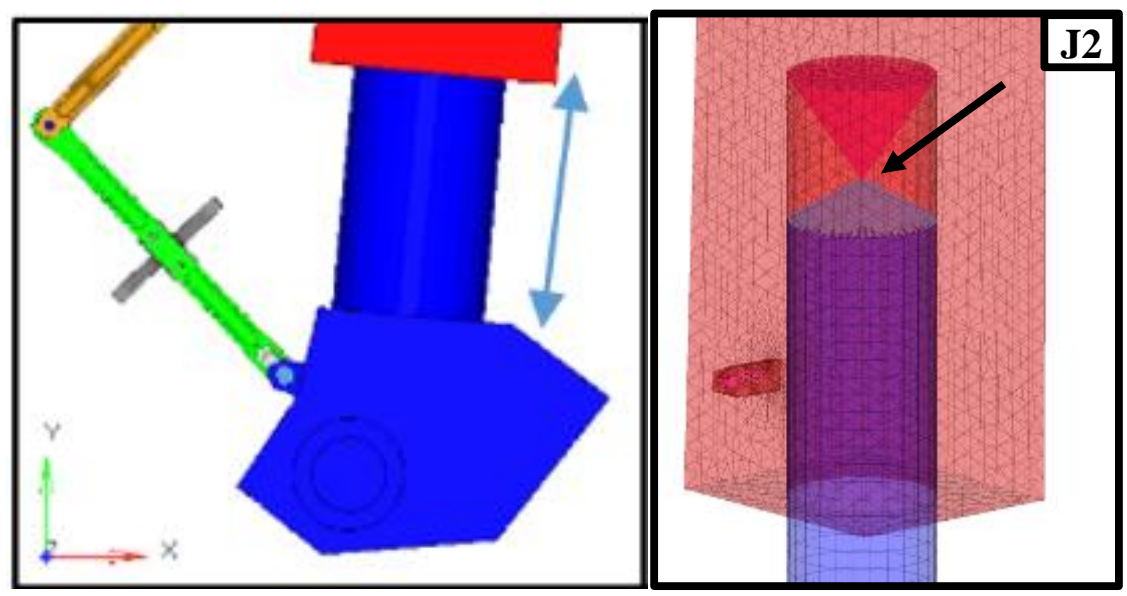

Figure 19 - Translational input motion $u(t)$ applied to the cylindrical number J2. 
For the rotational shimmy $\theta_{y}(t)$ and $\theta_{z}(t)$ gear walk motion, a mathematical equation for decaying oscillations was used and are as follow:

$$
\begin{aligned}
& \theta_{y}(t)=A_{2} e^{-\zeta_{2}\left(2 \pi f_{2}\right) t} \sin \left(2 \pi f_{2} t\right) \\
& \theta_{z}(t)=A_{3} e^{-\zeta_{3}\left(2 \pi f_{3}\right) t} \sin \left(2 \pi f_{3} t\right)
\end{aligned}
$$

where $A_{2}, A_{3}$ are the amplitudes of oscillation, $\zeta_{2}, \zeta_{3}$ are the damping ratio, and $f_{2}, f_{3}$ are the frequency of oscillation.

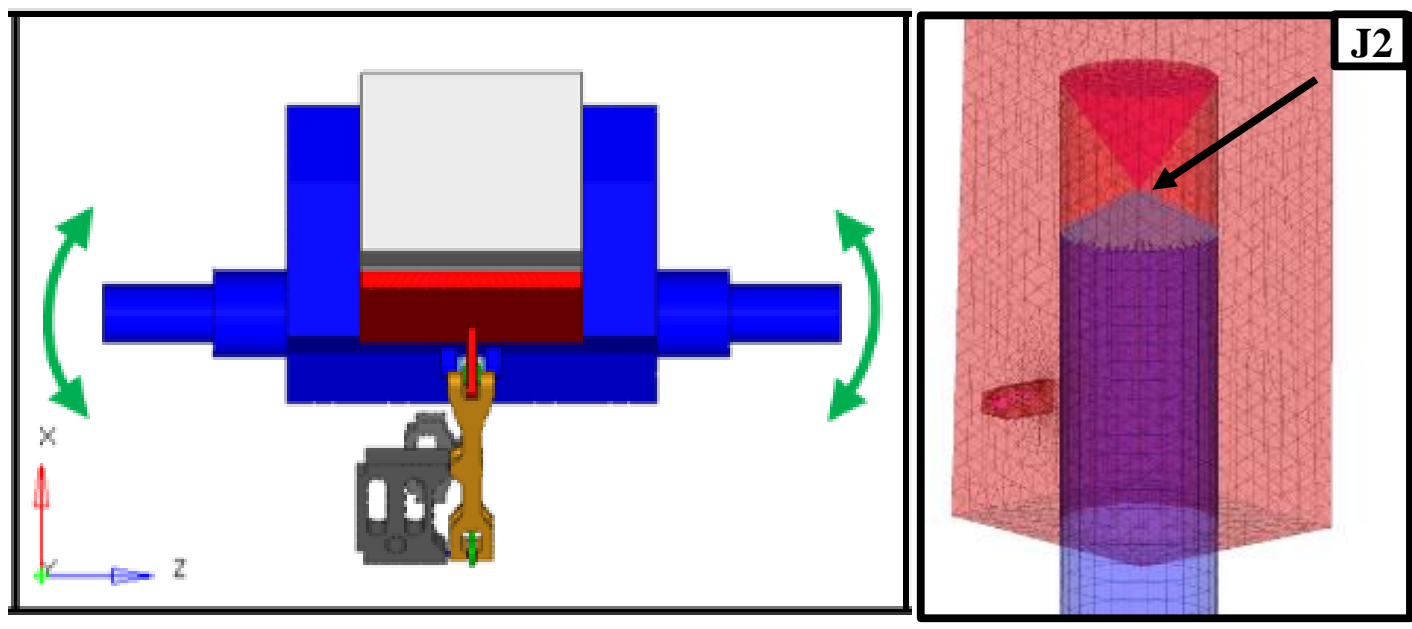

Figure 20 - Rotational Shimmy motion $\theta_{y}(t)$ applied to the cylindrical joint $\mathbf{J} 2$.

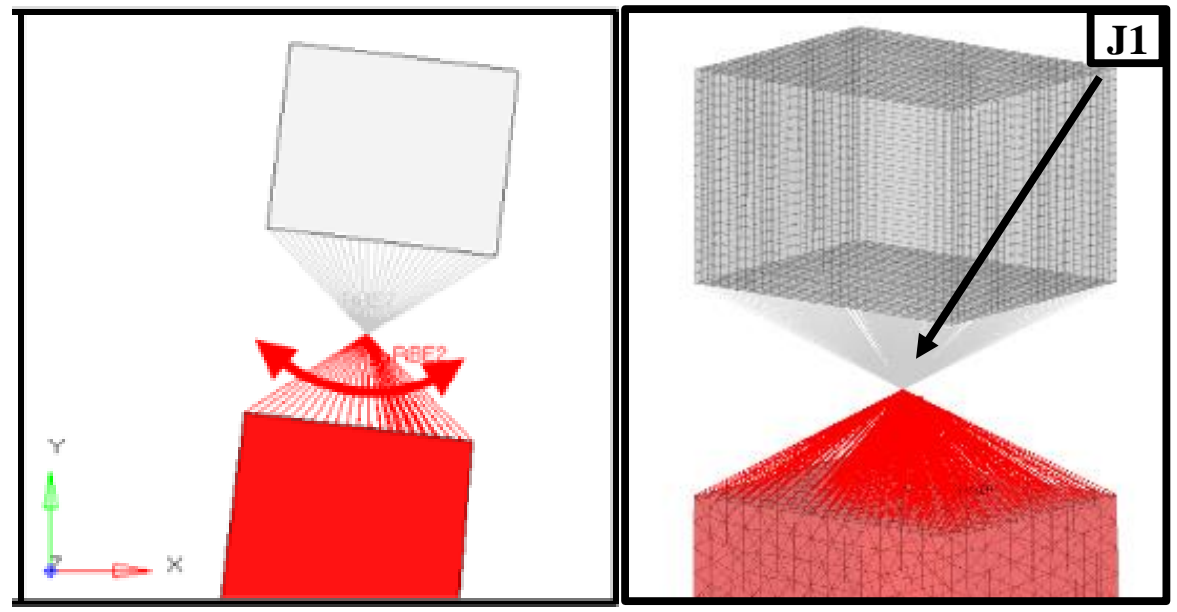

Figure 21 - Rotational gear walk motion $\theta_{z}(t)$ applied to the revolute joint $\mathbf{J 1}$. 
Based on a review of findings from AGARD [20] and Besselink [21], parameter values for the input motion were determined and shown in Table 3, with plots shown in Figure 22. Note that the units for angular displacement are shown as degrees for clarity, however these values were converted to radians in order to maintain unit consistency in the finite element analysis.

Table 3 - Parameter values for input motion.

\begin{tabular}{lll}
\hline Variable & Value & Units \\
\hline$A_{1}$ & 200 & {$[\mathrm{~mm}]$} \\
$f_{1}$ & 1 & {$[\mathrm{~Hz}]$} \\
$\zeta_{1}$ & 0.70 & {$[-]$} \\
$\beta$ & 0.7141 & {$[-]$} \\
$\theta$ & 0.7954 & {$[-]$} \\
$A_{2}$ & 2.5 & {$[\mathrm{deg}]$} \\
$f_{2}$ & 30 & {$[\mathrm{~Hz}]$} \\
$\zeta_{2}$ & 0.01 & {$[-]$} \\
$A_{3}$ & 0.1875 & {$[\mathrm{deg}]$} \\
$f_{3}$ & 30 & {$[\mathrm{~Hz}]$} \\
$\zeta_{3}$ & 0.01 & {$[-]$} \\
\hline
\end{tabular}



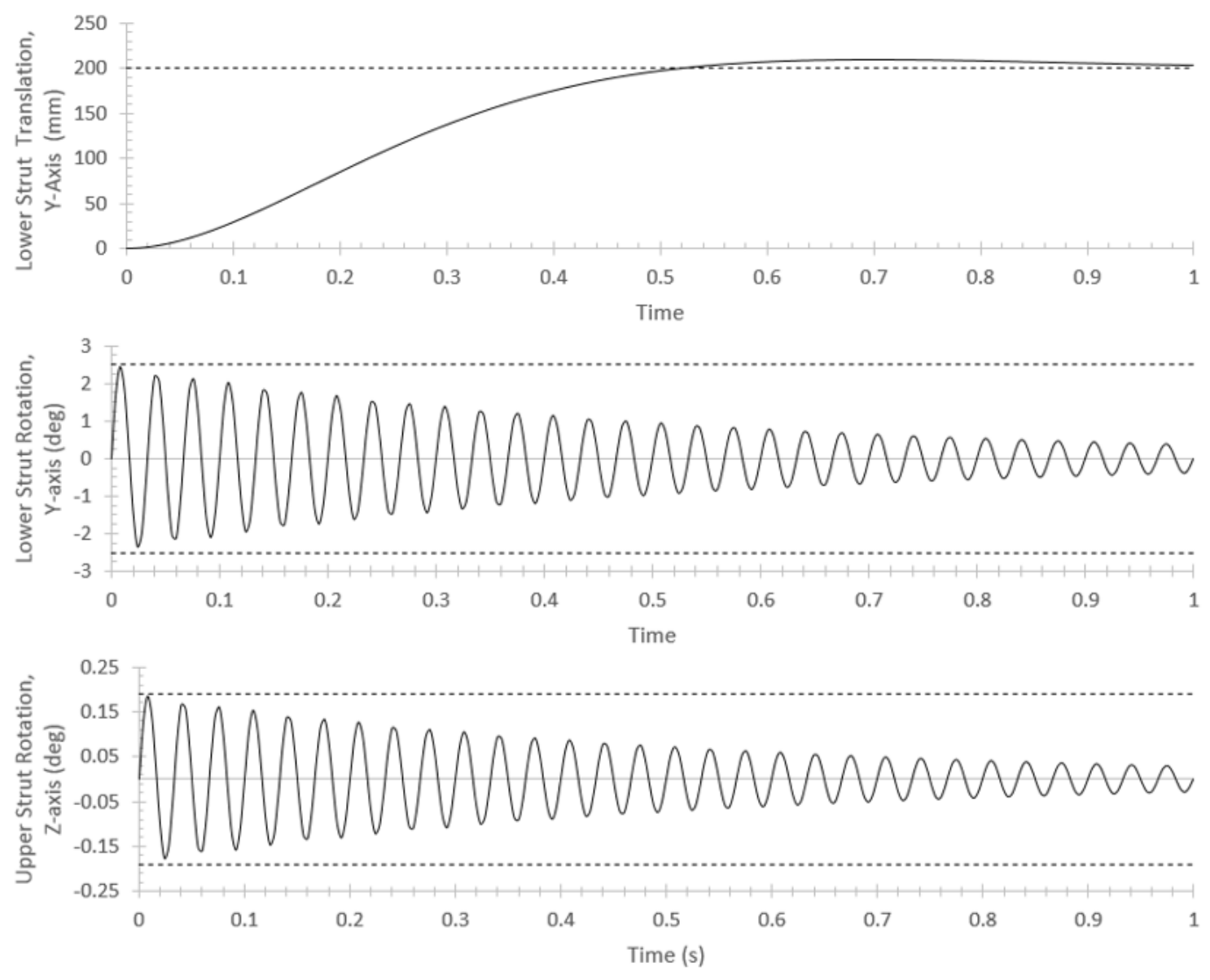

Figure 22 - Input motion functions for MBD analysis.

\subsection{Structural Behavior Characterization}

The next step in the modeling of the landing gear assembly was to define the body types of each component in the system. For an MBD analysis, there were three possible body definitions to prescribe: ground, rigid, and flexible. The ground definition was set to the ground component (shown in Figure 16) and remained fixed throughout the analysis. Rigid body definition was given to the upper and lower strut as their main purpose in the analysis was to transmit the input motion experienced during a landing load case into the slave link assembly and there was no interest in the structural behavior of these simplified components. The rest of the components, which include the slave links, cable guides, universal joint, and pins, were given a flexible body definition, as the structural aspects of these components will be analyzed. Table 4 summarizes the body definitions for each component in the finite element model, along with their corresponding material composition. 
Table 4 - Body definition for MBD Analysis

\begin{tabular}{ccc}
\hline Component & Material & Body Type \\
\hline Ground (Fixed) & N/A & Ground \\
Upper Strut & AISI 4340M & Rigid \\
Lower Strut & AISI 4340M & Rigid \\
Upper Slave Link & Al-7075-T73XXX & Flexible \\
Lower Slave Link & Al-7075-T73XXX & Flexible \\
Upper Cable Guide & Delrin 100 NC010 & Flexible \\
Lower Cable Guide & Delrin 100 NC010 & Flexible \\
Universal Joint & Al-7075-T73XXX & Flexible \\
Joint Pins & 17-4PH H1025 SS & Flexible
\end{tabular}

Linear isotropic material properties for steels and aluminum used in this model were obtained from ASM Handbooks [50] while the properties for the Delrin plastic were obtained from a supplier's datasheet [51]. Table 5 outlines the values for density, Poisson's ratio, Young's modulus, and yield strength of each material. Values for density and Young's modulus were reported in $\mathrm{kg} / \mathrm{m}^{3}$ and GPa rather than the unit consistency convention of $\mathrm{Mg} / \mathrm{mm}^{3}$ and $\mathrm{MPa}$, purely for the purpose of clarity. In the subsequent analysis and optimization, the correct units for density $\left(\mathrm{Mg} / \mathrm{mm}^{3}\right)$ and Young's Modulus (MPa) were used.

Table 5 - Material properties for components modeled.

\begin{tabular}{lcccc}
\hline \multicolumn{1}{c}{ Material } & $\begin{array}{c}\text { Density } \\
{\left[\mathrm{kg} / \mathrm{m}^{3}\right]}\end{array}$ & $\begin{array}{c}\text { Poisson's Ratio } \\
{[-]}\end{array}$ & $\begin{array}{c}\text { Young's Modulus } \\
{[\mathrm{GPa}]}\end{array}$ & $\begin{array}{c}\text { Yield Strength } \\
{[\mathrm{MPa}]}\end{array}$ \\
\hline AISI 4340M Steel & 7750 & 0.30 & 210 & 1586 \\
Al-7075-T73XXX & 2830 & 0.33 & 72 & 435 \\
17-4PH Stainless Steel & 7750 & 0.30 & 190 & 1172 \\
Delrin 100 NC010 & 1420 & 0.35 & 3.1 & 72 \\
\hline
\end{tabular}




\subsection{Manufacturing Process Characterization}

This research employs design optimization to introduce weight and cost savings to the slave link components. While a majority of the weight savings was realized through topology optimization, the cost savings for these components were realized by understanding the manufacturing processes behind producing these components and developing a methodology for evaluating the economics behind various machining operations. As previously mentioned, the slave link components are $\mathrm{CNC}$ machined from raw 7000-series aluminum stock. The justification behind using this manufacturing method was due to the production volume and scale of these components. Safran Landing System quoted that less than ten units are produced per month, therefore $\mathrm{CNC}$ machining was determined to be the most economically feasible option, relative to casting or extrusion processes.

The qualitative manufacturing process considerations for the slave link components are listed below. This list was developed with support from Safran Landing Systems and outline some of the key characteristics to take into account when developing a manufacturing cost model. They are as follows:

1. Raw material stock size selection

2. CNC milling setup and machining time

a. Drilling \& reaming holes

b. Pocketing \& contouring operations

c. Dimensional Tolerances

d. Filleting \& finishing passes

3. Machining Inspection

4. Anodizing for corrosion resistance

5. Quality control

6. Storage 
Based on the list of considerations, there are several aspects that are important to introducing cost savings, as they have reciprocating benefits. For instance, by altering the design in a manner where less raw material stock is required will inherently also lead to less machining to achieve the same amount of weight savings. Another instance of this reciprocating benefit can be realized in the reduction of machining operations which would consequently lead to less features for machining inspection and save time in the quality control process. Although the understanding of the cause and effect of manipulating these considerations for cost savings is important from a qualitative perspective, a quantitative model is essential to establish an objective comparison.

There are many challenges associated with developing an accurate and quantitative manufacturing cost model for a variety of reasons. Some of these challenges include the following:

1. Designs are subject to the interpretation of the machinist to manufacture

2. Machining time is highly influenced by the available machining facilities and resources

3. Order of operation in which features of a component is manufactured may change with different operators or programmers

4. Component cost estimation tools vary across different suppliers and exact details are usually kept confidential

In order to effectively create a quantitative cost model for manufacturing, the variances and uncertainties associated with the challenges described must be addressed. The methodology for overcoming these challenges was to normalize as much of the evaluation process as possible. Although this would lead to inaccuracies in terms of the actual cost of components, it would still be able to serve the purpose of determining relative changes to cost. For this research, the baseline design was chosen as the datum for comparison. Variations were eliminated by assuming that all components are manufactured with the same machining process, quality of tooling and machinist. For the purposes of this research, the assumptions made for the development of the manufacturing cost model were deemed sufficient and necessary to establish a means to quantitatively compare designs. 
A mathematical model for determining manufacturing cost was developed based on a weighted sum of normalized factors method and the governing equation is as follow:

$\mathrm{S}_{\mathrm{c}}=\sum_{i=1}^{N} w_{i} *\left(\frac{x_{i}^{A}}{x_{i}^{0}}\right)$

where $\mathrm{S}_{\mathrm{c}}$ is the cost score, $w_{i}$ is the weight for the $i$-th factor, $x_{i}^{0}$ is the $i$-th cost metric for the baseline design, $x_{i}^{A}$ is the $i$-th cost metric for the design being compared (design $\mathrm{A}$ ), and $N$ is the number of cost metrics being considered. Seven cost metrics were established from the list of considerations. The weightings for each metric was approximated with the aid and expertise of Safran Landing Systems internal cost estimation experience. It should be noted that the costs associated with anodizing and storage were not included in the development of this model, as the change in cost between the baseline and revised design was assumed to negligible for most cases. A summary of the cost metrics and weighting factors is shown in Table 6. Material volumes for stock and machining removal were determined using SolidWorks $\odot$, the other parameters were determined through manual inspection of the designs. This analysis was conducted after the revised design generation was performed.

Table 6 - Manufacturing cost model metrics and weight factors

\begin{tabular}{clcccc}
\hline \multirow{2}{*}{ Category } & \multicolumn{2}{c}{ Description } & \multicolumn{2}{c}{ Cost Metric } & \multicolumn{2}{c}{ Weighting Factor } \\
& & Variable & Units & Variable & Value [\%] \\
\hline Raw Material & Stock material volume & $x_{1}$ & {$[-]$} & $w_{1}$ & 10 \\
Machine & Number of stock flip operations & $x_{2}$ & {$[-]$} & $w_{2}$ & 15 \\
Setup & Number of milling tools & $x_{3}$ & {$[-]$} & $w_{3}$ & 10 \\
Machining & Material removal volume & $x_{4}$ & {$[-]$} & $w_{4}$ & 50 \\
& Number of pocketing operations & $x_{5}$ & {$[-]$} & $w_{5}$ & 5 \\
Inspection & Number of external contours & $x_{6}$ & {$[-]$} & $w_{6}$ & 5 \\
& Number of unique radii & $x_{7}$ & {$[-]$} & $w_{7}$ & 5 \\
\hline & & \multicolumn{2}{c}{ Total } & $w_{\text {Total }}$ & 100
\end{tabular}




\subsection{Chapter 5: Design Analysis and Optimization}

The second stage of this research involves performing both a high-fidelity MBD analysis and topology optimization of the slave link assembly model, created in the first stage. A process overview for the analysis and optimization will be covered in each respective section, followed by a set of parameters used to implement the various behavior characterizations previously defined. Results for the MBD analysis and topology optimization are also shown in this section, along with statistics regarding the computational cost and resources used to obtain them.

\subsection{Multi-Body Dynamic Analysis}

\subsubsection{Analysis Overview}

Transient MBD analysis was performed using OptiStruct@ [52]. The MBD solver within OptiStruct $\odot$ utilizes a combination of Differential Algebraic Equation (DAE) integrator [53] and Backward Differentiation Formula (BDF) method to solve the Euler-Lagrange representation of the equations of motion [54] and a simplified version for explanation purposes is represented as follow:

$M \ddot{U}_{D}+\Phi_{x}^{T} \lambda-f\left(U_{D}, \dot{U}_{D}\right)=0$

$\Phi\left(U_{D}, t\right)=0$

where $M$ represents the mass matrix, $\Phi$ is the displacement constraints vector, $f$ is the external force vector, $\lambda$ are the Lagrange Multipliers, and $U_{D}, \dot{U}_{D}, \ddot{U}_{D}$ are the calculated displacement, velocity, and acceleration vectors. Note that these equations meant to serve as a high level overview of the process and detailed information is available from Altair's theory manual [55]. This solver was selected because it was robust and computationally efficient. 
To introduce flexible bodies into the analysis, component mode synthesis (CMS) was performed on the finite element model with the flexible body definition. In the case of the slave link assembly, these components were defined in Table 4 of the previous section for the slave links, cable guides, and pins. The governing equation for CMS obtained from the OptiStruct $\odot$ theory manual [55] is represented in the following form:

$\hat{u}=\phi q$

where $\hat{u}$ is the displacements of the reduced structure, $\phi$ is a set of orthogonal modes, and $q$ is the matrix of modal participation factors. To clarify, the displacements are obtained from the MBD analysis and the modal participation factors are calculated from the CMS analysis. There are various methods for obtaining orthogonal modes. For the case of MBD analysis, the Craig-Bampton method [56] was used. This method utilizes a system of constrained and unconstrained mode shapes obtained by performing a series of normal mode and static structural analysis to determine orthogonal modes. Mathematically, a high level overview of this process may be described in the following manner:

Let $X_{N}$ and $D_{N}$ be the constrained mode shapes and eigenvalues obtained through a fully constrained normal mode analysis of a flexible body.

Then, let $X_{S}$ and $P_{S}$ be the displacements and joint forces associated with a series of unit displacement static structural analysis, performed for each joint degree of freedom.

From this, the mode shapes $X$ can be defined as

$X=\left[\begin{array}{l}X_{N} \\ X_{S}\end{array}\right]$

The reduced model stiffness $\widehat{K}$ and mass matrices $\widehat{M}$ can now be obtained by the following equations:

$\widehat{K}=\left[\begin{array}{cc}D_{c} & 0 \\ 0 & X_{S}^{T} P_{S}\end{array}\right]$

$\widehat{M}=X^{T} M X$ 
where $M$ is the mass matrix of the flexible body. Utilizing the $\widehat{K}$ and $\widehat{M}$ matrices, an eigenvector normalization problem may be formulated and solved to determine the orthogonal modes $\phi$.

$\widehat{K} N=\widehat{M} N D$

$\phi=X N$

where $N, D$ are the normal modes and eigenvalues determined from the eigenvector normalization problem.

\subsubsection{Analysis Parameters}

The parameters for the MBD analysis were set by enabling various control cards within the Optistruct@ solver and setting them to reflect the behavior characterizations defined in Sections 4.3 and 4.4. The material properties for each component was set using the MAT1 (linear isotropic material) control card with respect to the values and classifications defined in Table 4 and Table 5 . The number of normal modes was limited to 30 for the CMS analysis and set within the body definition interface for flexible bodies. To account for the effects of gravity in the analysis, the GRAV (gravity vector) control card was enabled and set to $9810 \mathrm{~mm} / \mathrm{s}^{2}$ in the negative Y-direction. The joint input motion equations from Section 4.3 were defined using the MBVAR (multi-body solver variable) control card and prescribed to the joint nodes with the MOTNGE (expression for grid point motion) control card. Finally, the time step and termination condition for the MBD analysis were set within the MBSIM (multi-body simulation selection) control card. To fully capture the oscillations from the landing load case without aliasing, the time step for the analysis was set to $0.004 \mathrm{~s}$ for a total duration of one second. 


\subsubsection{Analysis Results}

The MBD analysis was conducted on a Windows workstation computer (Intel i7-5820k, 12 cores $4.0 \mathrm{GHz}, 65431 \mathrm{MB}$ DDR4 RAM $2133 \mathrm{MHz}$ ), and the CPU (central processing unit) time was 0.95 hour. Surprisingly, it was discovered that a significant portion of the CPU time and resources was not spent on the solver but rather on the compiling and writing of the result files. Although the proportions vary between different analyses, it was observed that approximately $30 \%$ of the CPU time was spent on the CMS analysis, $10 \%$ on the MBD solver, and $60 \%$ on generating the output files. The read and write speeds of the storage used to run these analysis was also determined to have an influence on the rate at which the result files were generated.

The outputs of the analysis can be categorized into dynamic and structural responses. For this research, the output dynamic responses were classified as displacement, and acceleration results, while the output structural responses were classified as stress and strain results. To examine the dynamic response, displacement and acceleration plots for the upper and lower slave link at their corresponding center of gravities (CG) was generated. In terms of structural response, a stress contour for the upper and lower slave link was plotted at the time-step when the maximum von Mises stress occurs. Additionally, another plot was generated to show a stress history for the element which experiences the highest stress in each component through the simulation. An overview of these results is shown in Figure 23.

Compared to yield strength values from Table 5, the areas of high stress were still relatively low and unlikely to fail due to yielding. However from section 2.1.5, landing gear components are not typically designed around yield but rather around a minimum number of loading cycles $(60,000)$. For the 7000 -series aluminum used in the slave link components, the fatigue stress limit for 60,000 cycles is approximately $140 \mathrm{MPa}$ [57]. Therefore, the slave links were determined to have a safety factor of 5.6 and 6.4 for the lower and upper slave link, respectively. This suggested the two components were likely over-designed and increased the potential for weight and cost optimization. 

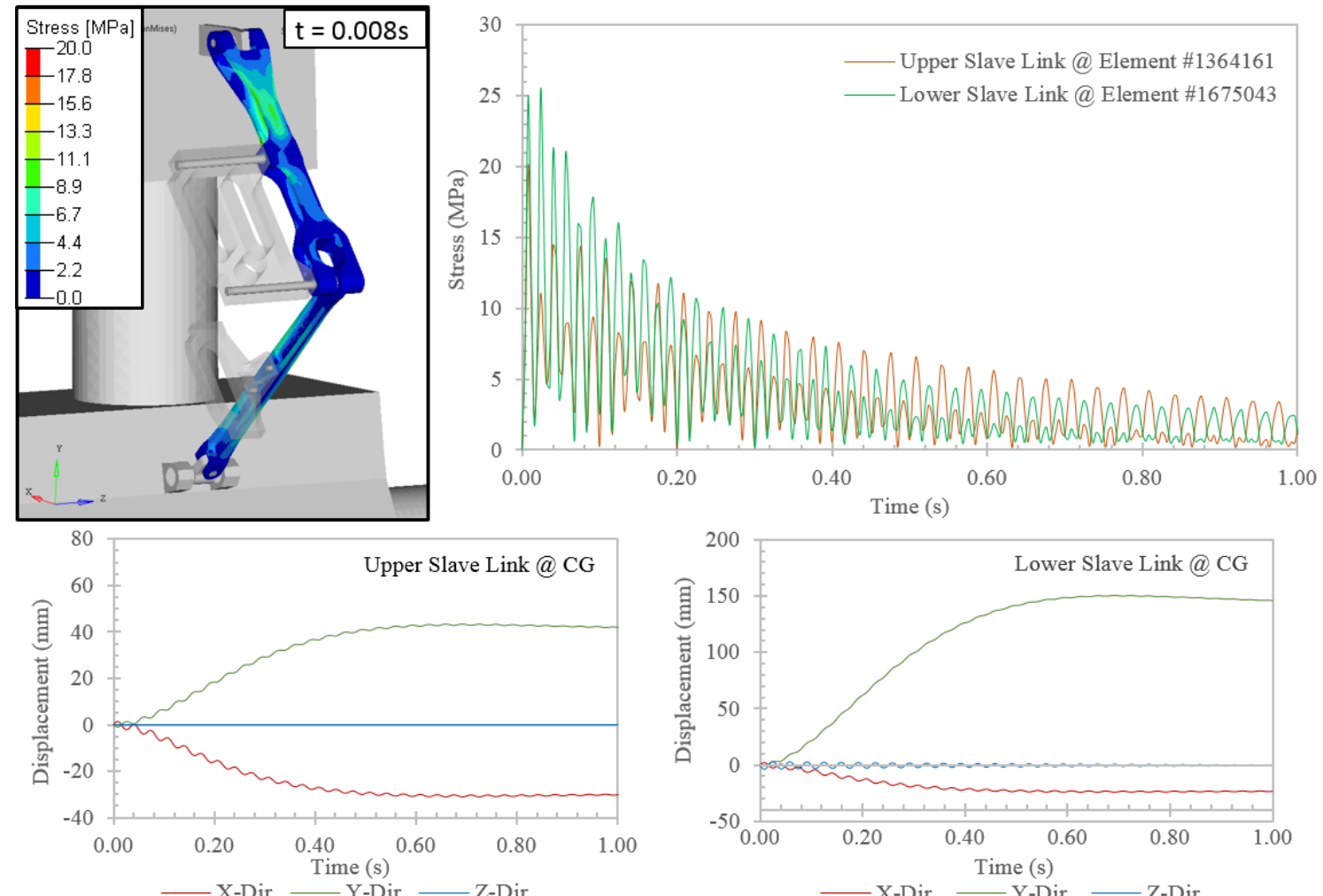

$\longrightarrow$ X-Dir — Y-Dir Z Z-Dir

- X-Dir — Y-Dir Z-Dir
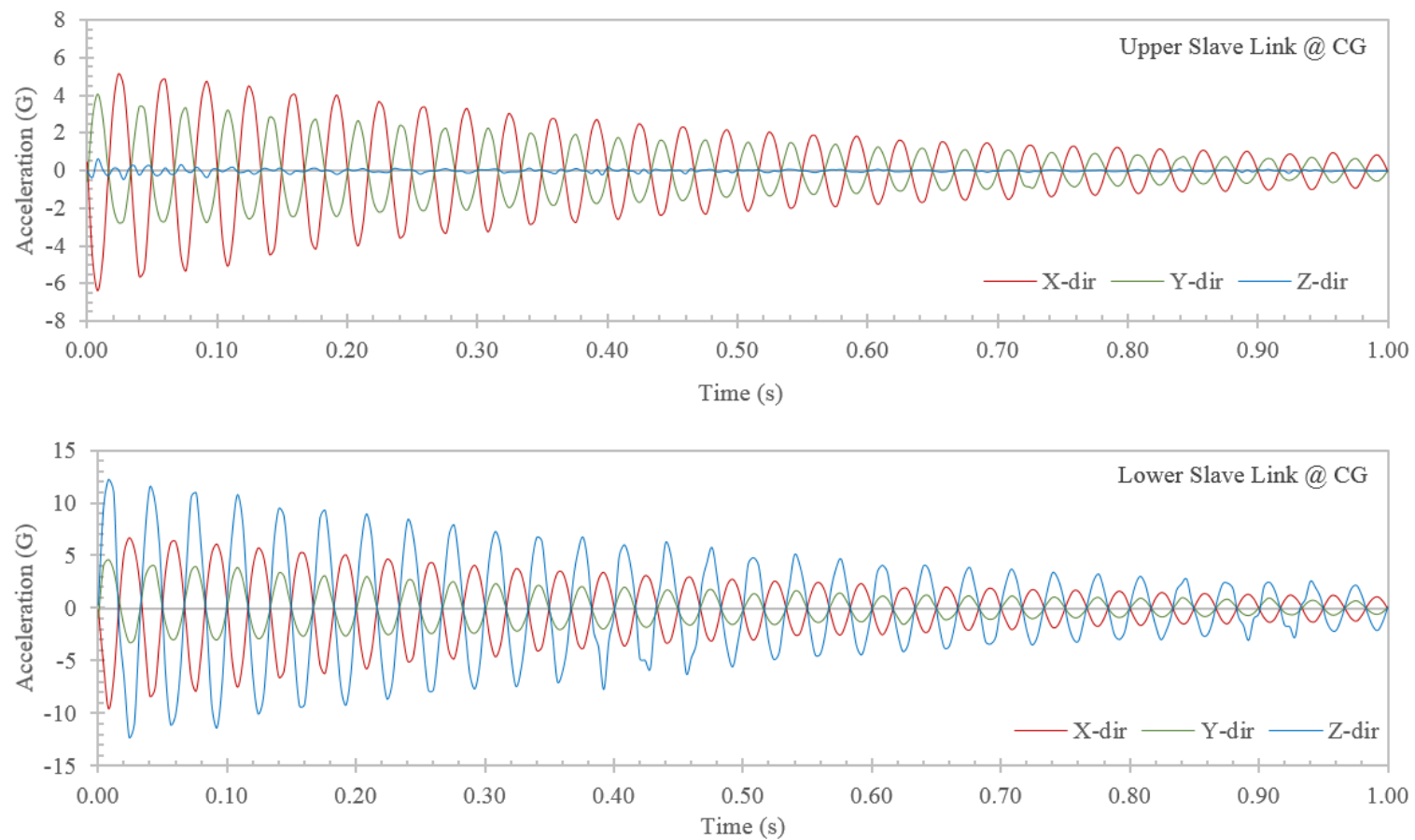

Figure 23 - Output from MBD analysis. 
Based on these results, it can be seen that the upper and lower slave link exhibit different responses in regards to which vibrational motion has the greater influence on the component. For the upper slave link, it can be seen that the rotational motion caused by gear walking induces the primary loading on this component, whereas the lower slave link is affected equally by both gear walk and shimmy vibrational loading. This response could have been anticipated based on the joint definitions prescribed to each component; given that the lower slave link was connected through a ball joint on one end and a universal joint on the other, the increased degrees of freedom explains the greater amount of excitation. However, it would have been more challenging to predict magnitudes of the response and highlight the benefits of conducting an MBD analysis.

From the stress plots, the highest stress for both components occurs at $0.008 \mathrm{~s}$, which corresponds to the first peak stress amplitude. Beyond this point, the stress continues to cycle and gradually diminish. Initially, the lower slave link exhibits higher stress relative to the upper slave link. At around $0.25 \mathrm{~s}$, this trend was inverted and the upper slave link began to experience higher stresses relative to the lower slave link past this point. The high stresses observed were due to the inertia of the cable guides acting on the slave link components. To clarify these observations, Figure 24 and Figure 25 were created to better visualize the observations made.

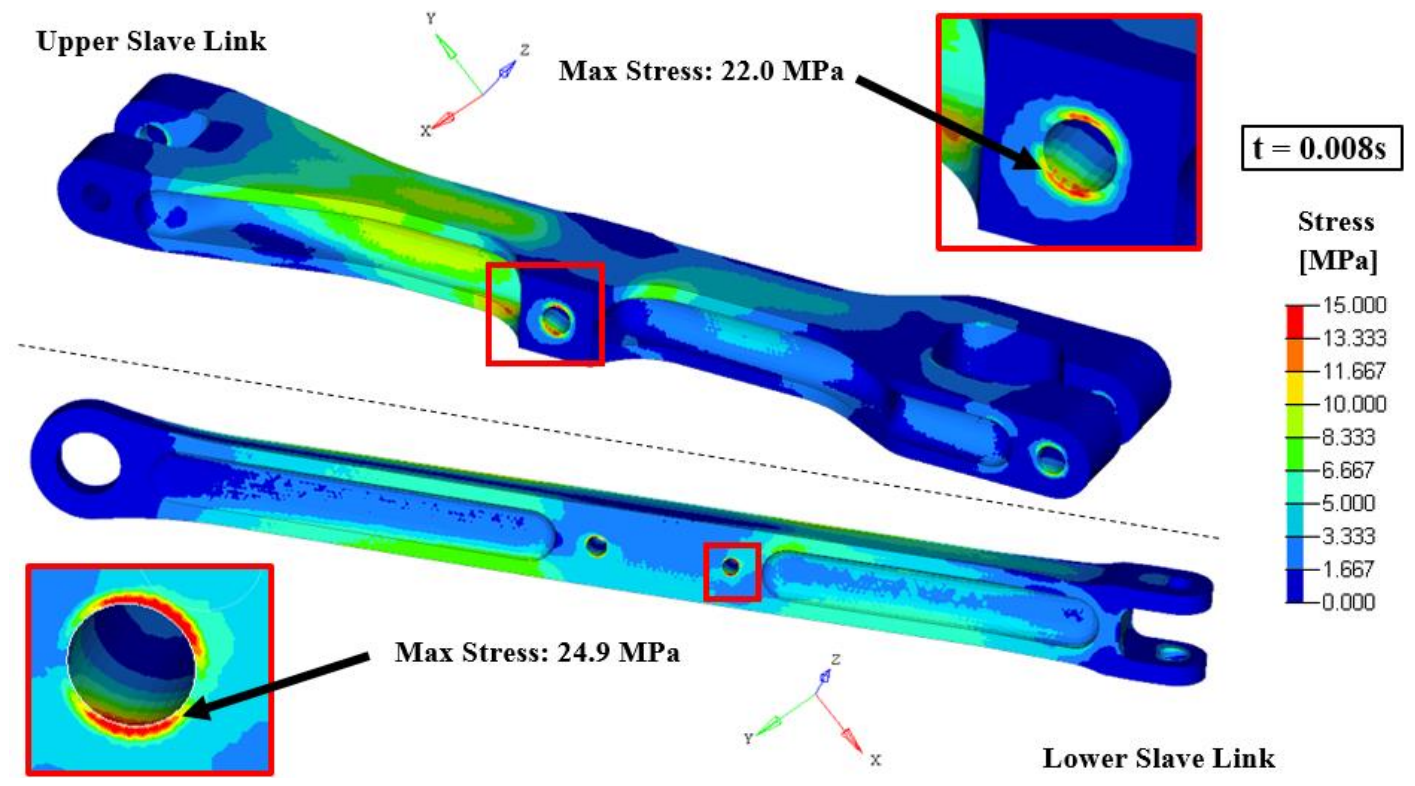

Figure 24 - Stress contours for baseline slave link components at $\mathbf{t}=\mathbf{0 . 0 0 8 s}$. 


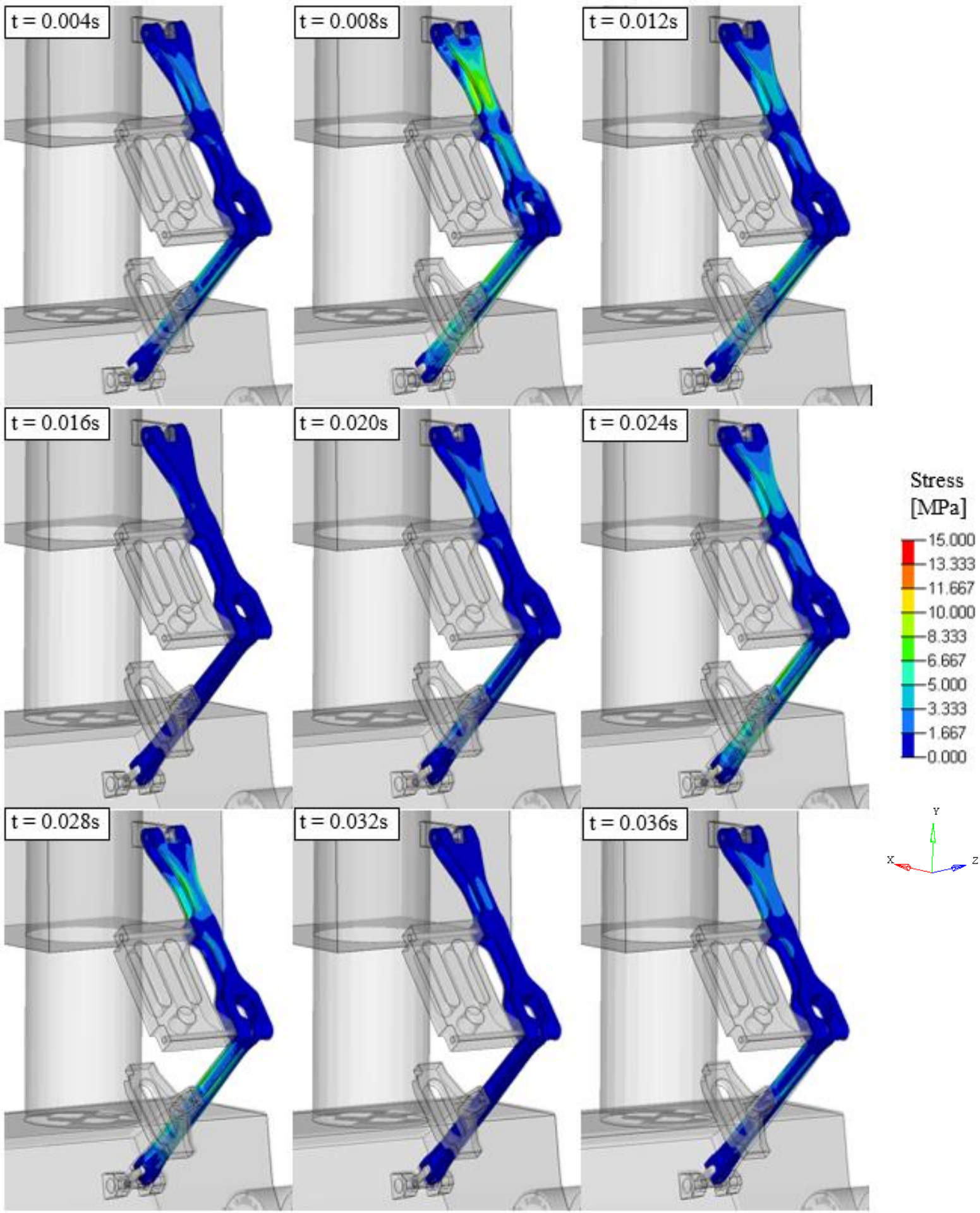

Figure 25 - Stress contours for slave link components for various time steps. 


\subsection{Topology Optimization}

\subsubsection{Optimization Overview}

The objective function of the topology optimization was to minimize compliance under dynamic loading determined from the MBD analysis. The optimization algorithm chosen in the Optistruct@ $\bigcirc$ solver was a dual optimizer based on separable convex approximation. In order to translate a time-based response into multiple load cases for topology optimization, the Equivalent Static Load Method (ESLM) developed by Park and implemented in Optistruct@ was utilized. Park has published several papers that thoroughly explain the methodology [46] and validations [58] performed on this technique. In essence, this process can be expressed mathematically in a simplified manner with the following equation:

$f_{e q}^{t}=K_{L}\left(\rho_{i}\right) U_{D}(t)$

where $f_{e q}^{t}$ is the calculated equivalent static loads vector at time step $t, K_{L}$ is the linear global stiffness matrix, $\rho_{i}$ is the density of the i-th element and $U_{D}$ is the dynamic analysis displacement vector at time $t$. To improve computational efficiency in this method, the solver systematically filters through each time step and only calculates equivalent loads where high compliance is observed. Although the exact methodology behind this filtering technique is not described in extensive detail in the theory manual, it should be noted that users have control over various settings of this filtering technique such as the maximum number of loads to extract. These parameters will be further covered in Section 5.2.3.

The MBD topology optimization problem of the slave link assembly components can be described with the following mathematical statement:

minimize $C\left(\rho_{i}^{(k)}\right)$

subject to $\left\{\begin{array}{c}K_{L}\left(\rho_{i}{ }^{(k)}\right) U_{L}^{t}=f_{e q}^{t} \\ \sum_{i=1}^{N} \rho_{i}{ }^{(k)} v_{i}-V^{*} \leq 0 \\ 0<\rho_{\min } \leq \rho_{i}{ }^{(k)} \leq 1 \\ \rho_{i}{ }^{(k)}=g_{j}\left(d_{i}\right)\end{array}\right.$ 
where the objective is to minimize the compliance $C$ under dynamic loading, $\rho_{i}$ represents the element density of the $i$-th design variable $d_{i}$ for topology optimization. $U_{L}^{t}$ and $f_{e q}^{t}$ are nodal displacement and equivalent static loads vectors at time step $t$, respectively, and $K_{L}$ is the linear global stiffness matrix. $k$ identifies the optimization outerloop iteration and is part of the ESLM. The purpose of this outerloop is to update the equivalent static loads $f_{e q}^{t}$ as the design domain evolves through the course of the optimization for a more accurate representation of loads experienced by the updated structure. $V^{*}$ denotes the volume fraction constraint, and $v_{i}$ is the element volume of the $i$-th design variable. $\rho_{\min }$ is the minimum allowable density to prevent the occurrence of numerical instabilities in the optimization. Lastly, $g_{j}$ represents the $j$-th manufacturing, such as draw and extrusion, or symmetry constraint function imposed on the design space to drive the optimal solution towards a more readily manufacturable result.

In Optistruct $\odot$, there are three conditions that will cause the optimization to terminate and output results. The first condition is when the maximum number of innerloop design iterations is reached. This condition is controlled by the DESMAX control card within the design optimization parameters. The value for DESMAX varies depending on other optimization parameter values and will be discussed in Section 5.2.3. For the purposes of explaining this process, let $N$ be the maximum number of design iterations. The second condition occurs when the maximum number of outerloop iterations $k$ is reached. This condition controlled by the ESLMAX control card and is set to 30. The third condition for termination is when an optimal solution has been achieved and the follow optimality criteria has been met:

$\left\|\rho_{i}^{(k)}-\rho_{i}^{(k-1)}\right\| \leq \varepsilon$

where $\varepsilon$ is the convergence criterion set by the OBJTOL control card and the default value being 0.005. An optimization process overview flow chart is shown in Figure 26. 


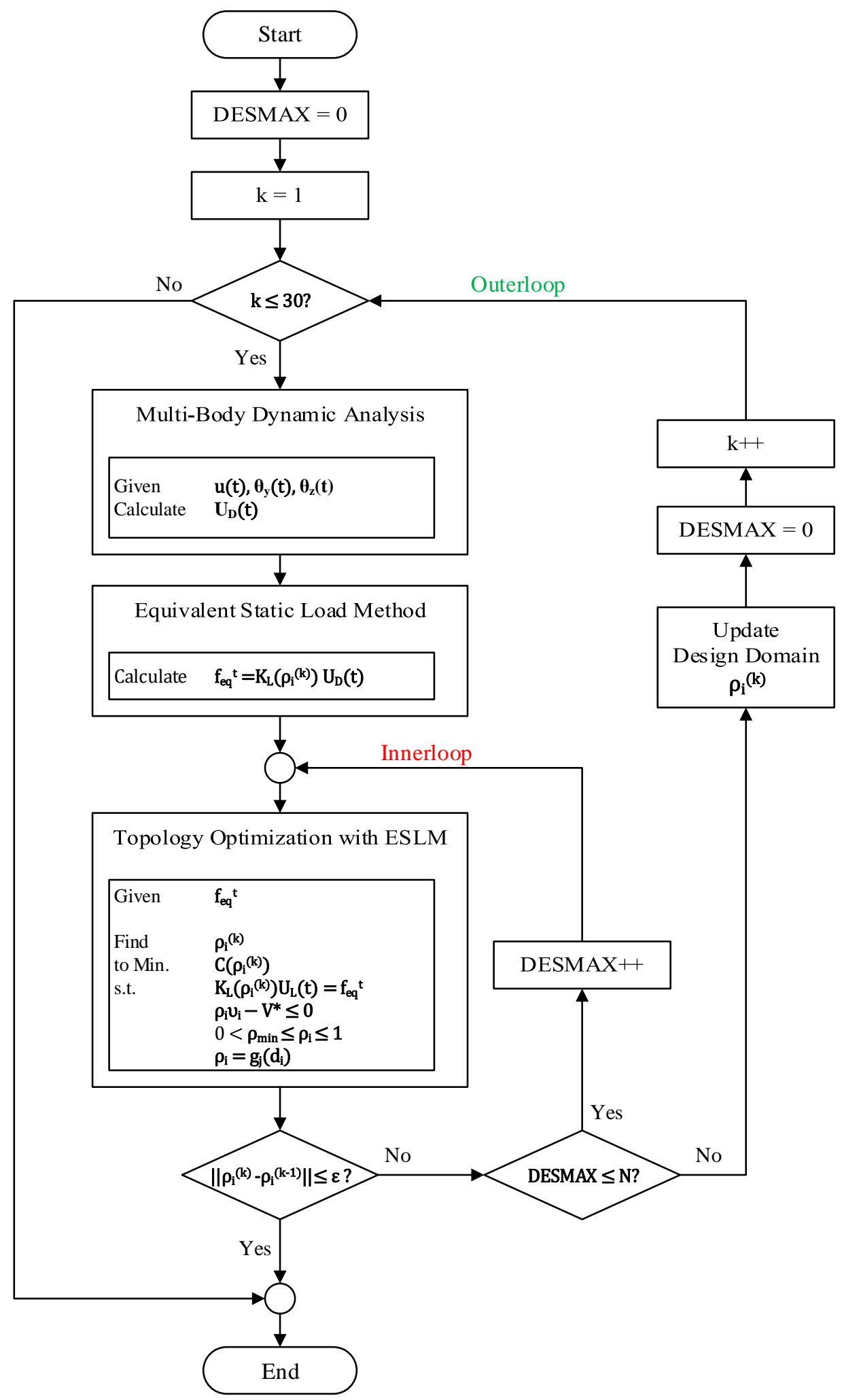

Figure 26 - MBD topology optimization overview flowchart. 


\subsubsection{Design Space Generation}

With the MBD analysis successfully implemented, the landing gear model was ready to be reconfigured to perform topology optimization. The first step to reconfiguring the model was to generate a solid design space based on the original upper and lower slave link CAD geometries by de-featuring the model to create a uniform domain.

Certain cutouts, such as the clearance cutout between the upper and lower slave link joint connection, were left unchanged in order to prevent interference between each component when loaded. Since there are various locations where connections have to be made to connect the design space to joints, a ring of elements was designated as a non-designable space, meaning these elements are not altered throughout the optimization. This ensures the joints are always properly connected to the component and prevent any numerical instabilities from occurring. The generated design spaces are shown in Figure 27.

The upper slave link design space was segmented into two separate design spaces because preliminary topology optimization runs resulted in elements accumulating solely in design space B while design space A remained virtually void. Upon closer inspection, it was determined that the cause of this behavior was due to the difference in compliance caused by the presence of the upper cable guide. The stiffness from the cable guide was the reason that no solid elements were generated in design space A. The solution implemented to mitigate this phenomenon was to optimize with only one design space active at a time. This resolved issues for later optimization runs, and optimization parameters will be further discussed in the subsequent section. 


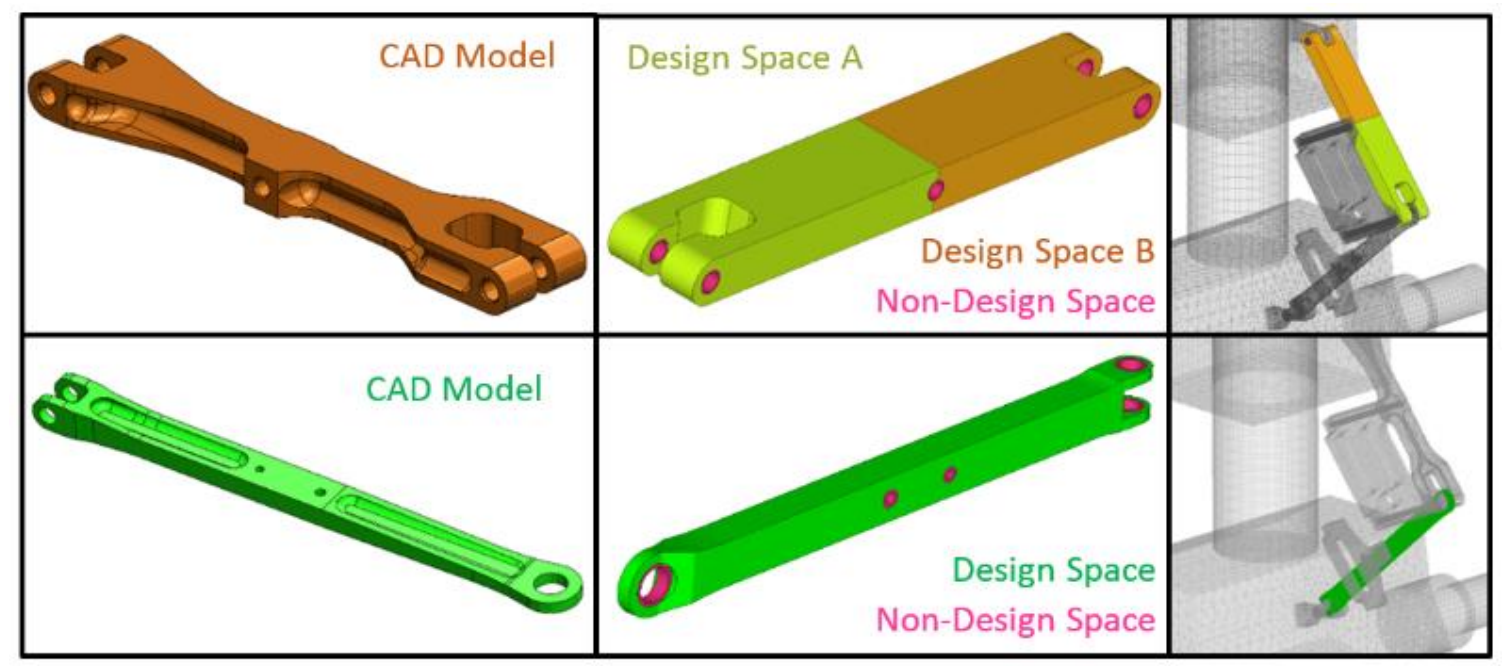

Figure 27 - Design space generation.

\subsubsection{Optimization Parameters}

For density-based topology optimization, solid isotropic microstructure penalization (SIMP) method, developed by Bendsøe and Sigmund [59], is used to enhance the discretizing of the underlying relaxed mixed integer non-linear problem (MINLP) and forcing intermediate element densities to converge towards either a 0 (void element) or 1 (solid element). This is done using the following penalization scheme:

$E=\rho_{i}^{P} E_{0}$

where $E$ represents the penalized Young's modulus of the $i$-th element, $E_{0}$ is the Young's modulus of the material, and $P$ is the penalty factor. For all optimization runs, $P$ is set to 3 in the DISCRETE control card.

As mentioned in Section 5.2.1, Optistruct $\subset$ has several control cards to vary the ESLM load filtering and selection. For this research, these parameters were set to the following:

- $\quad$ ESLSOPT - Time step screening strategy toggle. (Value = $1 \mathrm{ON})$

- $\quad$ ESLSTOL - Screening tolerance $($ Value $=0.3)$

- ESLMAX - Maximum number of outerloop iterations, $k($ Value $=30)$ 
To drive the solution towards a feasible design, control parameters such as checkerboard and minimum member size filters [60], manufacturing constraints and symmetry planes [61] were used. These optimization settings are controlled by the following control cards and summarized in the list below:

- CHECKER - Checkboard filter $($ Value $=1 \mathrm{ON})$

- $\quad$ MATINIT - Initial material density of design domain $($ Value $=0.9)$

- OPTMETH - Optimization algorithm $($ Value = DUAL $)$

- OBJTOL - Convergence criterion $\varepsilon($ Value $=0.05)$

- DESMAX - Maximum number of innerloop design iterations

$\circ \quad($ Value $=30$ or 80 if MINDIM is defined $)$

- $\quad$ MINDIM - Minimum member size $($ Value $=$ See Table 7$)$

- $\quad$ TYP - Symmetry pattern constraint $($ Value $=$ See Table 7$)$

- $\quad$ DRAW - Manufacturing constraint $($ Value $=$ See Table 7)

Multiple optimization runs were performed with different control parameter settings and volume fraction targets. To establish a baseline for a comparison of the effects in manipulating certain parameter settings, an unconstrained topology optimization run with no control parameters enabled was done for each design space. All topology optimization runs were conducted on a Windows workstation computer (Intel i7-5820k, 12 cores $4.0 \mathrm{GHz}, 65431$ MB DDR4 RAM 2133 $\mathrm{MHz}$ ) and the CPU time for each run was recorded. These settings and CPU times are listed in Table 7. 
Table 7 - Optimization parameters, settings, and computational cost

\begin{tabular}{|c|c|c|c|c|c|c|c|c|}
\hline Design Space & Run ID & $\begin{array}{c}\text { Volume } \\
\text { Fraction } \\
\left(V^{*}\right)\end{array}$ & $\begin{array}{l}\text { Manufacturing } \\
\text { Constraint } \\
\text { (DRAW) }\end{array}$ & $\begin{array}{c}\text { Symmetry } \\
\text { Constraint } \\
\text { (TYP) }\end{array}$ & $\begin{array}{l}\text { Minimum } \\
\text { Member Size } \\
\text { (MINDIM) }\end{array}$ & $\begin{array}{l}\text { CPU Time } \\
\text { [hour] }\end{array}$ & $\begin{array}{l}\text { MBD ESLM } \\
\text { Outerloops }(k)\end{array}$ & $\begin{array}{l}\text { Total Innerloop } \\
\text { Design Iterations* }\end{array}$ \\
\hline \multirow[t]{3}{*}{ Upper Slave Link (A) } & R1 & 0.20 & N/A & N/A & N/A & 15.70 & 4 & 41 \\
\hline & $\mathrm{R} 2$ & 0.20 & Split Draw & 1 plane & 3 & 30.30 & 4 & 73 \\
\hline & $\mathrm{R} 3$ & 0.20 & Split Draw & 2 plane & 3 & 27.13 & 4 & 76 \\
\hline \multirow[t]{3}{*}{ Upper Slave Link (B) } & R4 & 0.20 & N/A & N/A & N/A & 19.30 & 4 & 37 \\
\hline & R5 & 0.20 & Split Draw & 1 plane & 3 & 56.75 & 5 & 92 \\
\hline & R6 & 0.20 & Split Draw & 2 plane & 3 & 46.12 & 6 & 106 \\
\hline \multirow[t]{3}{*}{ Lower Slave Link } & R7 & 0.25 & N/A & N/A & N/A & 7.92 & 4 & 41 \\
\hline & R8 & 0.20 & Split Draw & 1 plane & 3 & 10.18 & 3 & 55 \\
\hline & R9 & 0.25 & Split Draw & 2 plane & 3 & 13.43 & 3 & 55 \\
\hline
\end{tabular}

* - The total number of innerloop design iterations is calculated across all ESLM outerloops. 


\subsubsection{Optimization Results}

Figure 28 shows an overview of all the topology optimization results generated for the upper slave link design space A (R1-R3), upper slave link design space B (R4-R6), and lower slave link (R7-R9). These results were generated in Altair's post-processing software HyperView $\bigodot$, with a density threshold set to 0.505 for the isosurface results of R1 to R3, and 0.755 for R4 to R9. These parameter settings were chosen as they best exemplify the load paths for each optimization run. The red regions signify areas of high element density close to 1.000 , yellow regions signify element densities around 0.755 , and green regions signify densities around 0.505 .

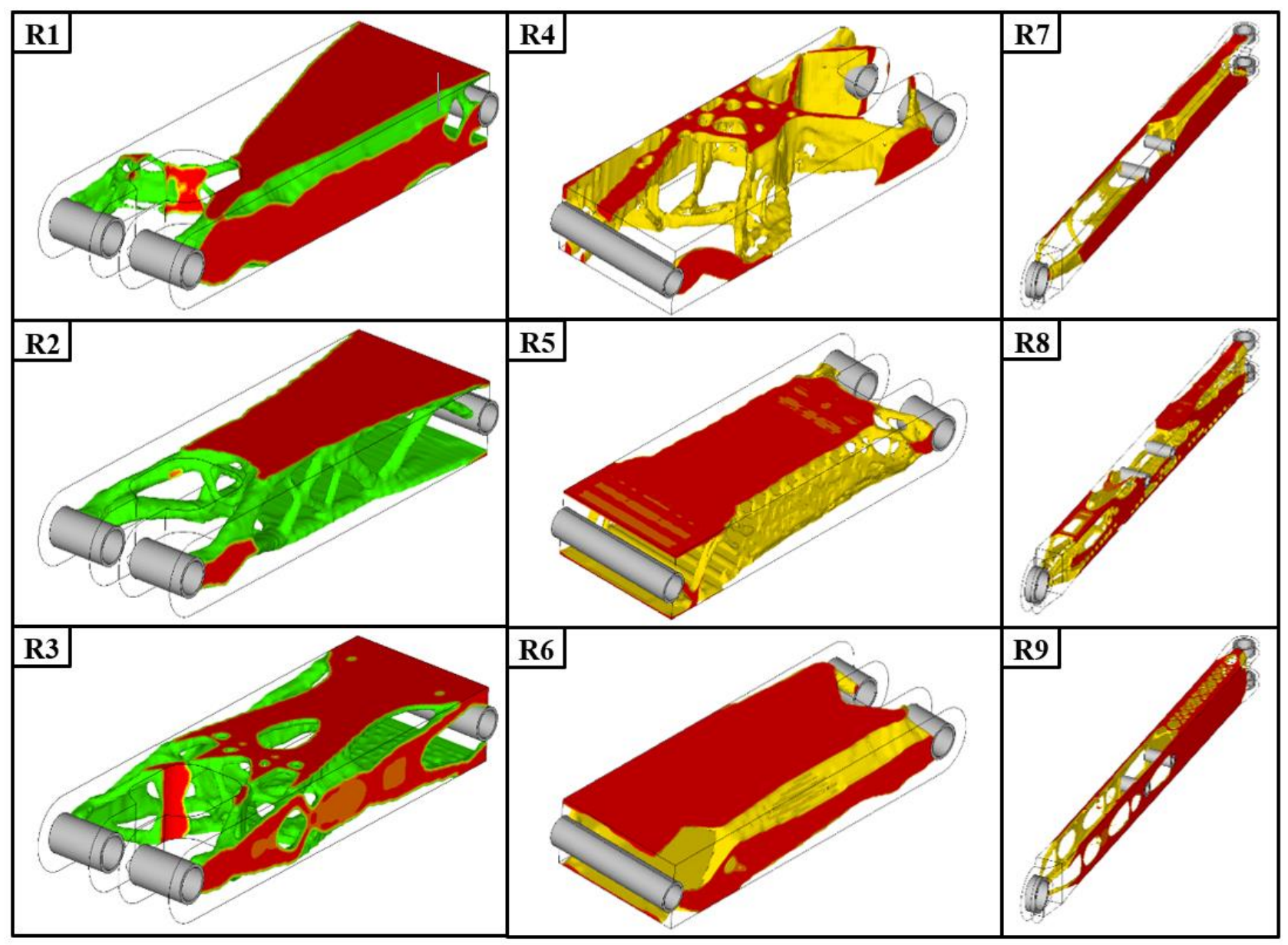

Figure 28 - Topology Optimization Results. 
Once all runs were completed, objective values for compliance at every iteration were recorded in an output text files for each optimization run. At the time of conducting this research, there were no built-in features within Optistruct $\odot$ that allowed for the extraction of these values to observe the convergence behavior of each optimization. To overcome this challenge, a custom MATLAB $\odot$ parser code was written to read and extract the necessary information needed to build the convergence plots. A portion of the output file and the MATLABC code used was included in Appendix A and B, respectively. Convergence plots generated are shown in Figure 29. 

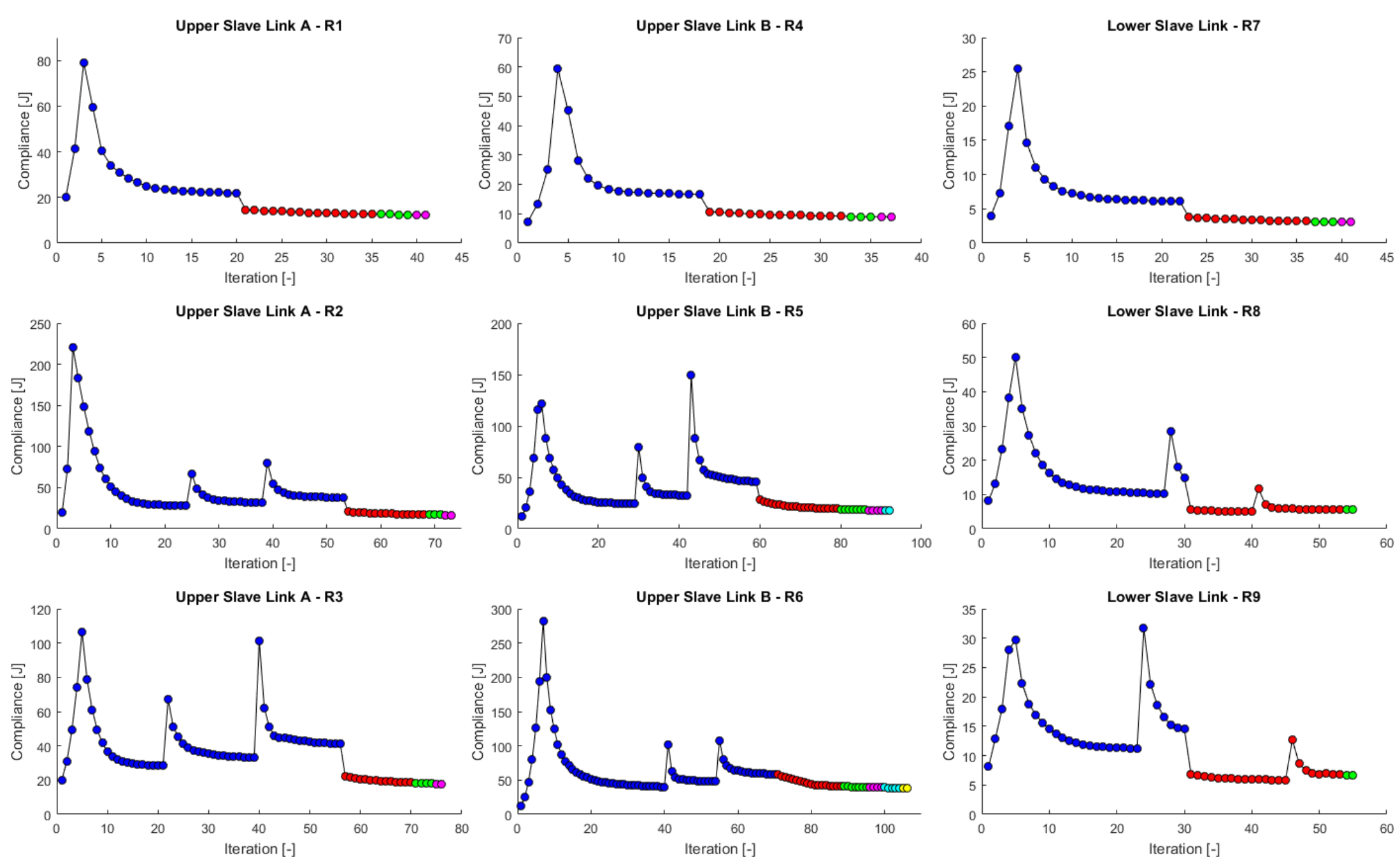

- Outerloop $1 \bullet$ Outerloop $2 \bigcirc$ Outerloop $3 \bigcirc$ Outerloop $4 \bigcirc$ Outerloop $5 \bigcirc$ Outerloop 6

Figure 29 - Optimization Convergence Plots. 
Without any manufacturing and symmetry constraints (R1, R4, and R7), the optimization converges relatively quickly and the profile was as one would expect. The expected optimization behavior is an initial increase in compliance as a result of the volume constraint being violated. Once the volume constraint has been satisfied, the structure was gradually refined until either an optimal solution or the maximum number of design iterations is achieved, whichever occurs first. In the other optimization runs, there were three noticeable spikes in compliance followed by a gradual convergence at the end. This behavior was attributed to how OptiStruct handles and implements manufacturing and symmetry constraints.

As seen from the plots R3 and R9, a phenomenon that was caused by the presence of the outerloop updating scheme is observed. Since the loads experienced changes with the geometry of the structure, outerloops play a necessary step in recalculating the equivalent static loads of the updated geometry for improved accuracy in the optimization. The results in the abrupt changes in compliance that is typically observed during the transition from outerloop 1 to 2 . It is important to note that this phenomenon is only seen in MBD topology optimization and not in traditional linearstatic topology optimization.
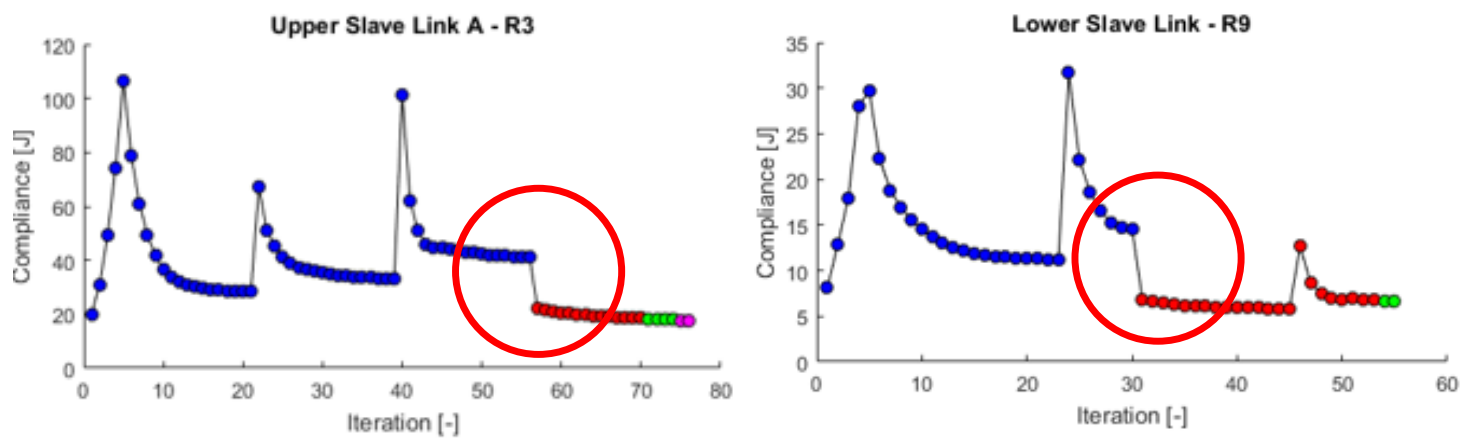

Figure 30 - Abrupt changes in compliance due to ESLM outerloop recalculation of loads

Another interesting trend was the transition of subsequent outerloops was typically observed to be smooth. This would suggest the topology of the design domain is not changing drastically during this outerloop transition, and as a result, the changes in the recalculated loads are observed to be negligible. 


\subsection{Chapter 6: Detailed Design Reinterpretation}

Based on the findings from multiple topology optimization results from

Figure 28, there were certain commonalities and differences that can be used as insights towards developing an optimal design. In runs where no manufacturing or symmetry constraints were imposed (R1, R4 and R7), the structures formed had an organic nature in their formation, with no clear method for reproducing the results with traditional manufacturing techniques. Another unique behavior exhibited by these results was that areas of high densities typically occurred near the boundaries of the design space. This seems to suggest the area moment of inertia has a noticeable impact on the stiffness of the structure and was a key design insight to utilize.

It is common for unconstrained topology optimization results to give some indication for the primary load path within a structure. However, this was not the case for the results generated from MBD topology optimization and a potential reason for this behavior may be explained by the oscillating nature of the loading experienced. Since the load cases extracted were at various instances of the oscillation cycle, multiple load paths may have been superimposed and yielded the results observed.

For results R2, R5, and R8, a split draw manufacturing constraint and single plane of symmetry was activated during these runs, which shows some promising insights for contouring and cutout locations. Again, all results exhibited a hollow structure behavior; however, R2 and R8 also show resemblances of a truss structures with various cutouts.

Lastly, results R3, R6, and R9, a split draw constraint along with 2 planes of symmetry were used to constrain the design space towards a feasible design. The results followed a similar hollow structure behavior, but the distribution of cutouts and truss structures did change in terms of location, size, and shape. 
To realize and subsequently validate the results obtained from MBD topology optimization, isosurfaces from runs R2, R6, and R8 were chosen as the template to be used to generate revised designs. Even though the other results were not directly used as templates in the design generation, a significant amount of design insights were drawn and indirectly influenced various design decisions throughout the reinterpretation stage.

Once the results were selected, isosurfaces from HyperView@ $@$ were exported as STL (stereo lithography) files and reimported into SolidWorks@ for CAD modeling. In order to have a better visualization of the boundaries of the design space as well as to ensure the pinhole locations of the revised design match the existing baseline, the imported isosurfaces were projected onto the existing CAD models of the design space for both the upper and lower slave link.

\subsection{Revised Design Generation}

Two different approaches were taken when generating designs from the topology isosurfaces. The first approach for Revised Design \#1 (RD\#1) involved a close replication of the features present in the topology results as possible with little consideration of manufacturability and cost. Therefore, a design based upon the use of rectangular tubing, bushing inserts, laser cutting, and sheet metal operations as the manufacturing inspiration was created. Bushings are used in areas where pins are to be inserted, as they will increase the contact area. Although it is possible to manufacture this design, it would likely be infeasible due to the high degree of complexity and cost associated with producing such a component. Nonetheless, this design was produced to demonstrate the full potential benefits in terms of weight and performance that can be achieved directly from the raw topology results. Compared with the baseline the design, RD\#1 yielded weight savings of $60 \%$ and $70 \%$, for the upper and lower slave link, respectively. 


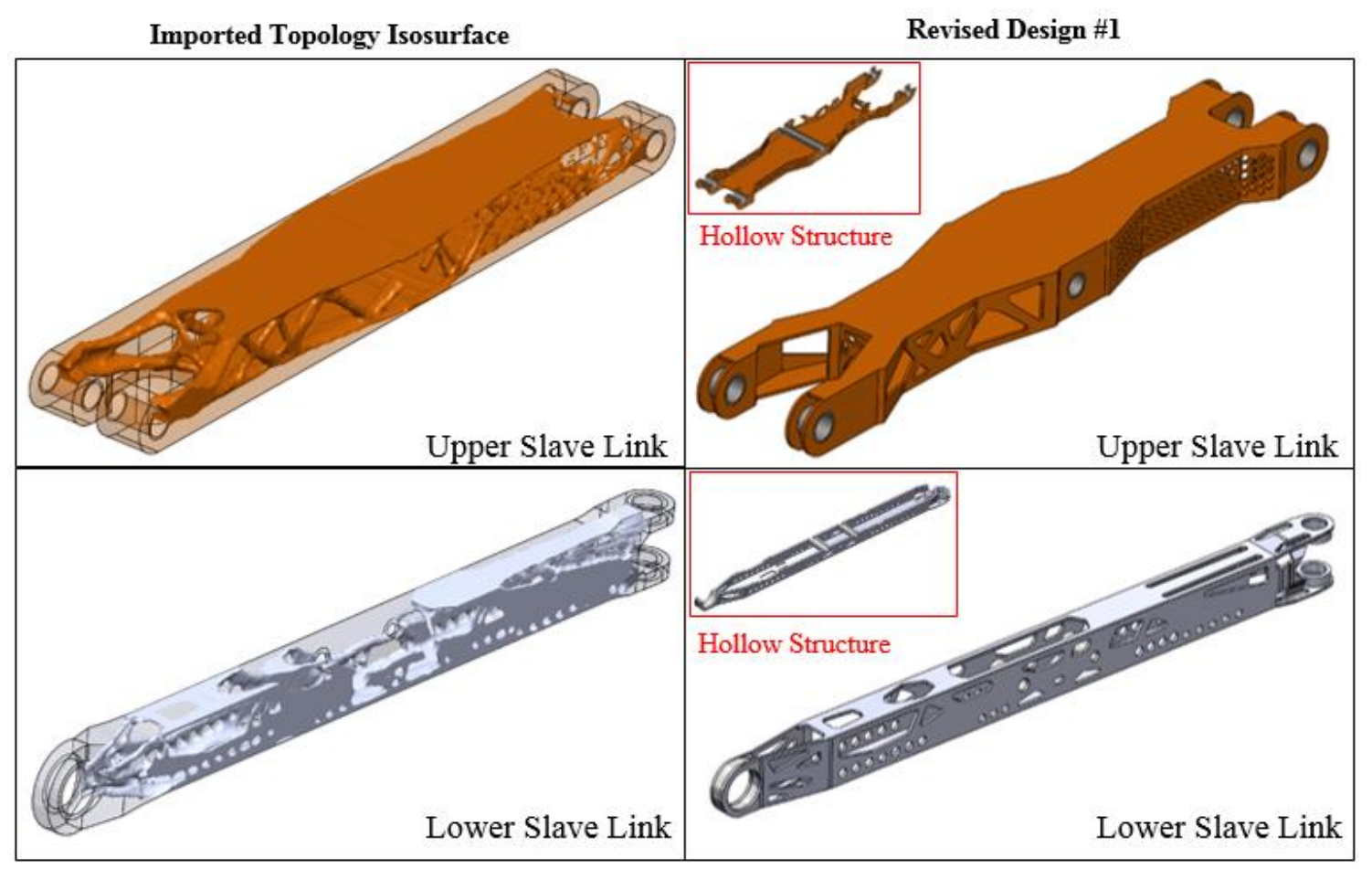

Figure 31 - RD\#1 interpreted from topology isosurface results

The second approach for Revised Design \#2 (RD\#2) in design generation focused on creating a design that improved on the manufacturability and cost savings of the baseline design, with some consideration for weight savings whenever possible. Collaboration with Safran Landing Systems has indicated there are often cases where the importance and initiatives for cost savings outweigh the benefits of weight savings; it was strongly advised to also develop designs with this consideration in mind. The baseline design were manufactured using CNC milling of a block of aluminum with several drilling, pocketing, and contouring operations. Constrained by manufacturing processes outline in Section 4.5, the generated designs would likely have to deviate from the isosurfaces of the topology results, as it would be both infeasible and impractical to manufacture all features present in the result. This should not be interpreted as a limitation of this methodology, but rather as a limitation of our current manufacturing technology to feasibly realize these mathematically optimized structures. 
In an attempt to simultaneously introduce both weight and cost savings with current manufacturing methods, various iterations for a practical design were created. Variations between design iterations were determined heuristically, with the goal of realizing the reciprocating benefits described in Section 4.5. This was accomplished through design and manufacturing experience as well as insights drawn from the computational results produced. Once all design iterations were modelled, a manufacturing cost analysis utilizing metrics developed from this research was performed to determine the most cost efficient design. The results of this analysis are summarized in Appendix C.

From this analysis, it was ultimately determined that the most efficient method for realizing reciprocating benefits in manufacturing cost was by reducing the stock material volume required. By reducing the stock volume, the amount of material removal required to achieve the target weight savings also decreased, leading to fewer machining operations required for producing the component. This technique was implemented for the redesign of the upper slave link, as it was deemed to be over-conservative, considering the loads it experiences. This claim was justified by the results obtained from the MBD analysis and topology optimization results. Since the lower slave link was already an efficient structure for handling the loads seen in the MBD analysis, it was determined that there were minimal design changes that could effectively introduce both weight and cost savings. As such, minor alterations to certain dimensions and introducing a slot feature in place of a pocketing feature allowed for weight savings to be introduced without additional cost. This outcome was deemed acceptable and CAD models generated for RD\#2 and the corresponding manufacturing cost analysis are shown in Figure 32 and Table 8. 


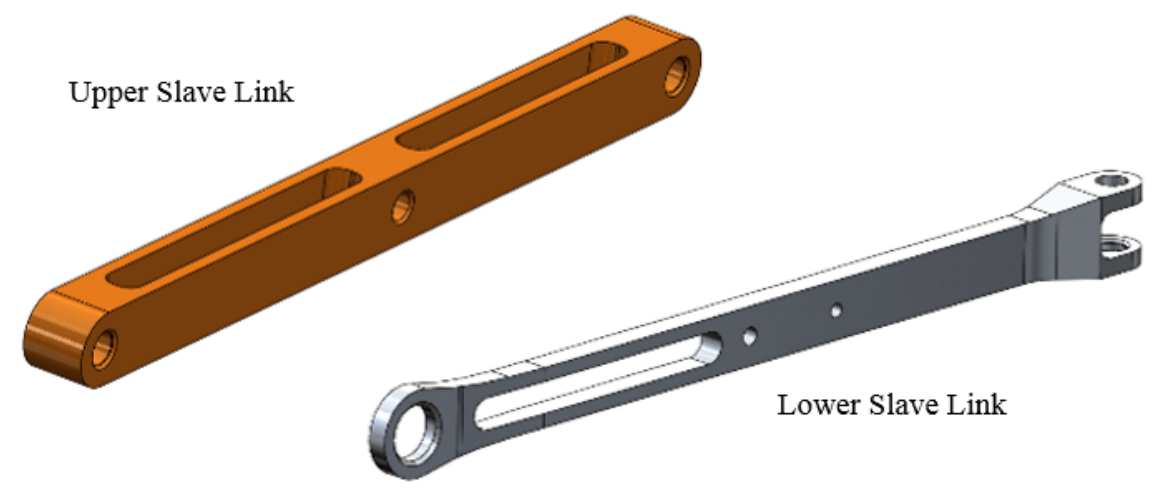

Figure 32 - Final CAD models for RD\#2.

Compared to the baseline design, the optimized design, RD\#2, yielded weight savings of $41 \%$ and $24 \%$, for the upper and lower slave link. Through the use of metrics developed for this research, the predicted cost savings for the upper and lower slave link were determined to be $37 \%$ and 3\%, respectively. When RD\#2 was proposed to Safran Landing Systems, interest was shown in determining accurate cost estimates for these components. As a result, detailed drawings were created for the RD\#2 design and sent to Safran Landing Systems to conduct a component manufacturing cost estimation. Although details were not disclosed, the estimations yielded potential cost savings of approximately $60 \%$ with $50 \%$ reduction in machining time, relative to the baseline. These detailed drawings are shown in Appendix D.

Table 8 - RD\#2 manufacturing cost analysis.

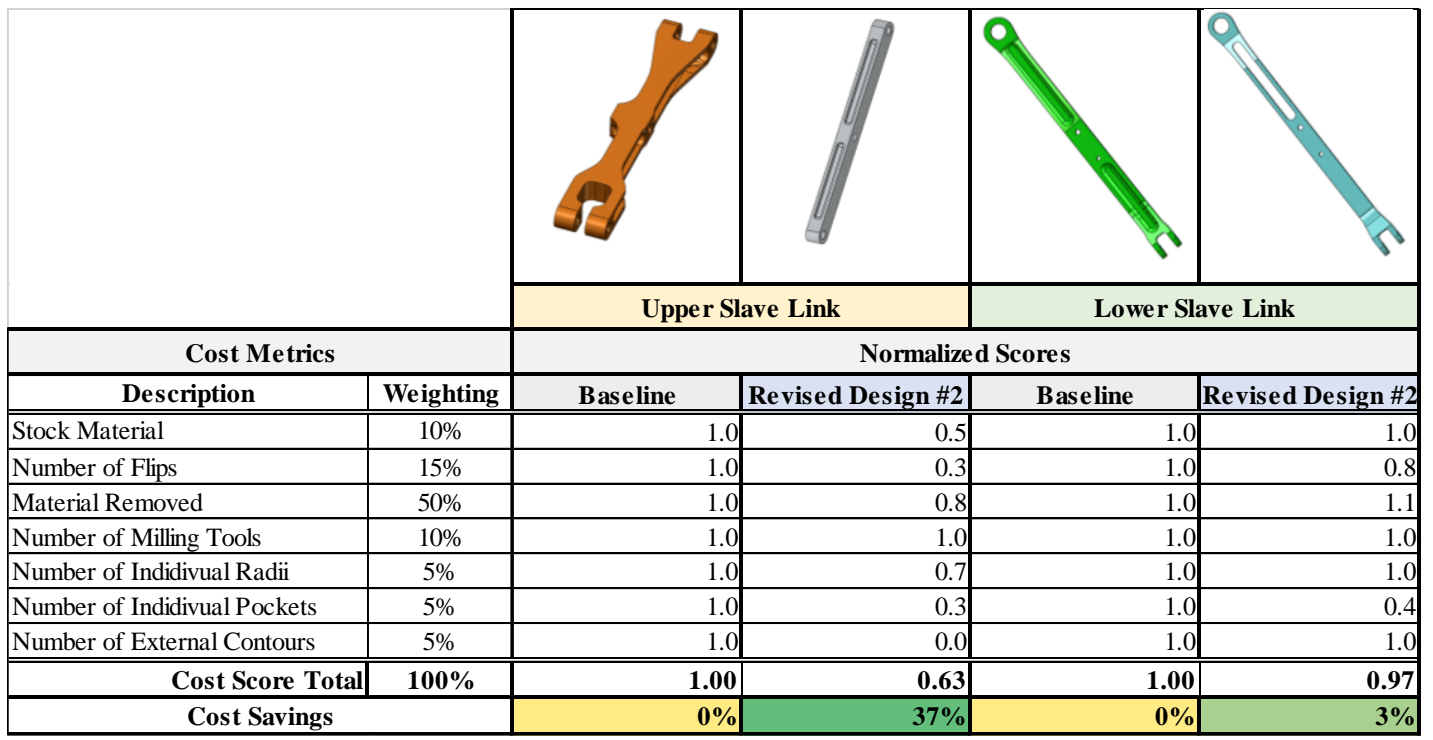




\subsection{Design Performance Evaluation}

The CAD models of the revised designs were exported from SolidWorks $@$ and brought into the existing finite element model for the baseline slave link assembly. The new components were meshed and joint definitions were redefined accordingly, as was done with the baseline model setup. Once the models were reconfigured to accept RD\#1 and RD\#2, MBD analysis was once again performed and a comparison of the dynamic and structural responses was made. The models for the MBD analysis are found in Figure 33. For the dynamic response, acceleration and displacement plots were generated for each revised design, shown in Figure 34. For the structural response, a comparative stress versus time plot between the revised and baseline designs was created, along with a stress contour for each design at time step 0.008s. This time step was chosen because it corresponds to when the maximum von Mises stress was induced for both slave link components. The stress contours and plots for the elements that experience the peak stresses are shown in Figure 35. Finally, detailed stress contours were generated to better observe and visualize the peak stresses at time step 0.008s. These are shown in Figure 36 for RD\#1 and Figure 37 for $\mathrm{RD \# 2.}$

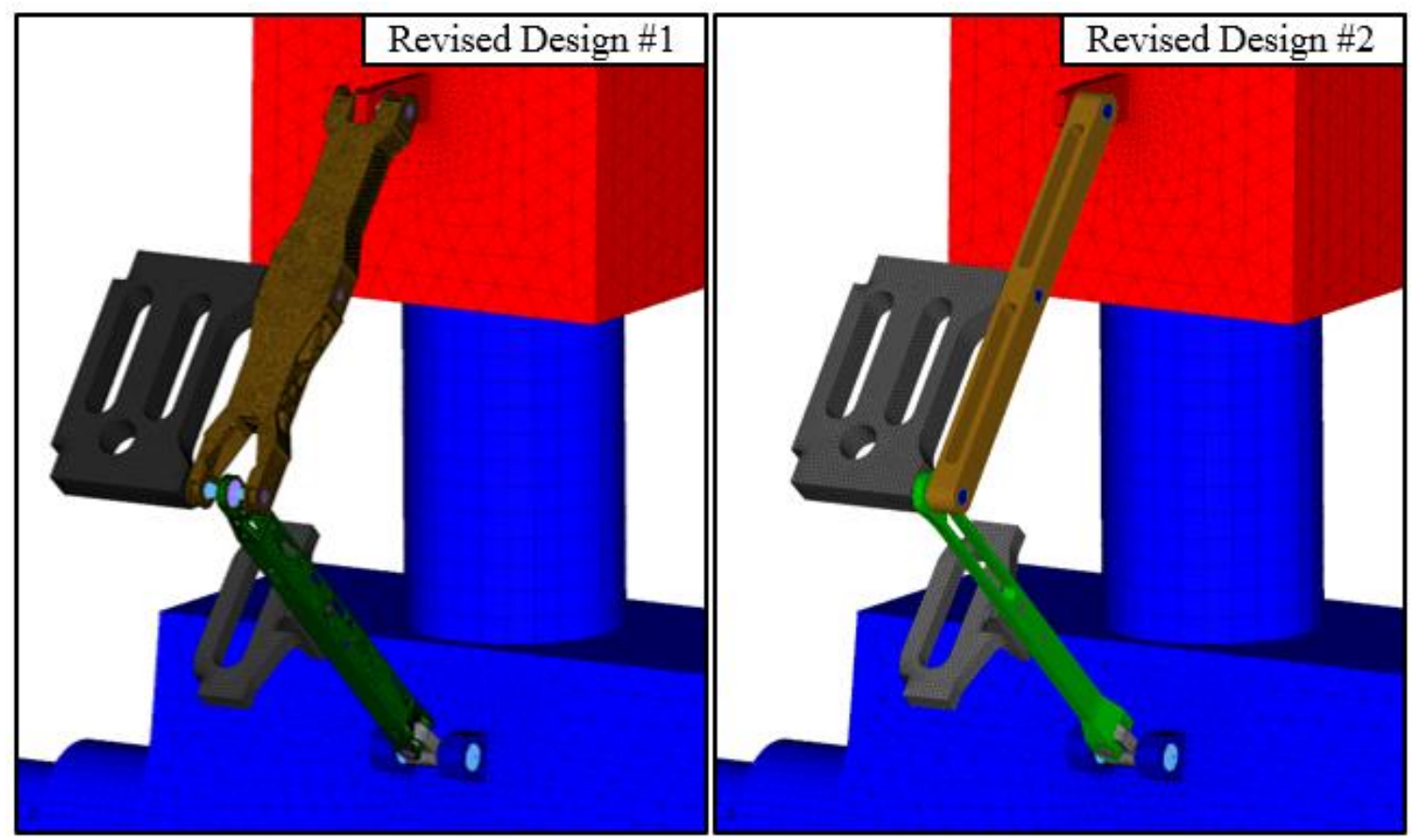


Figure 33 - Reconfigured finite element models for the revised designs.
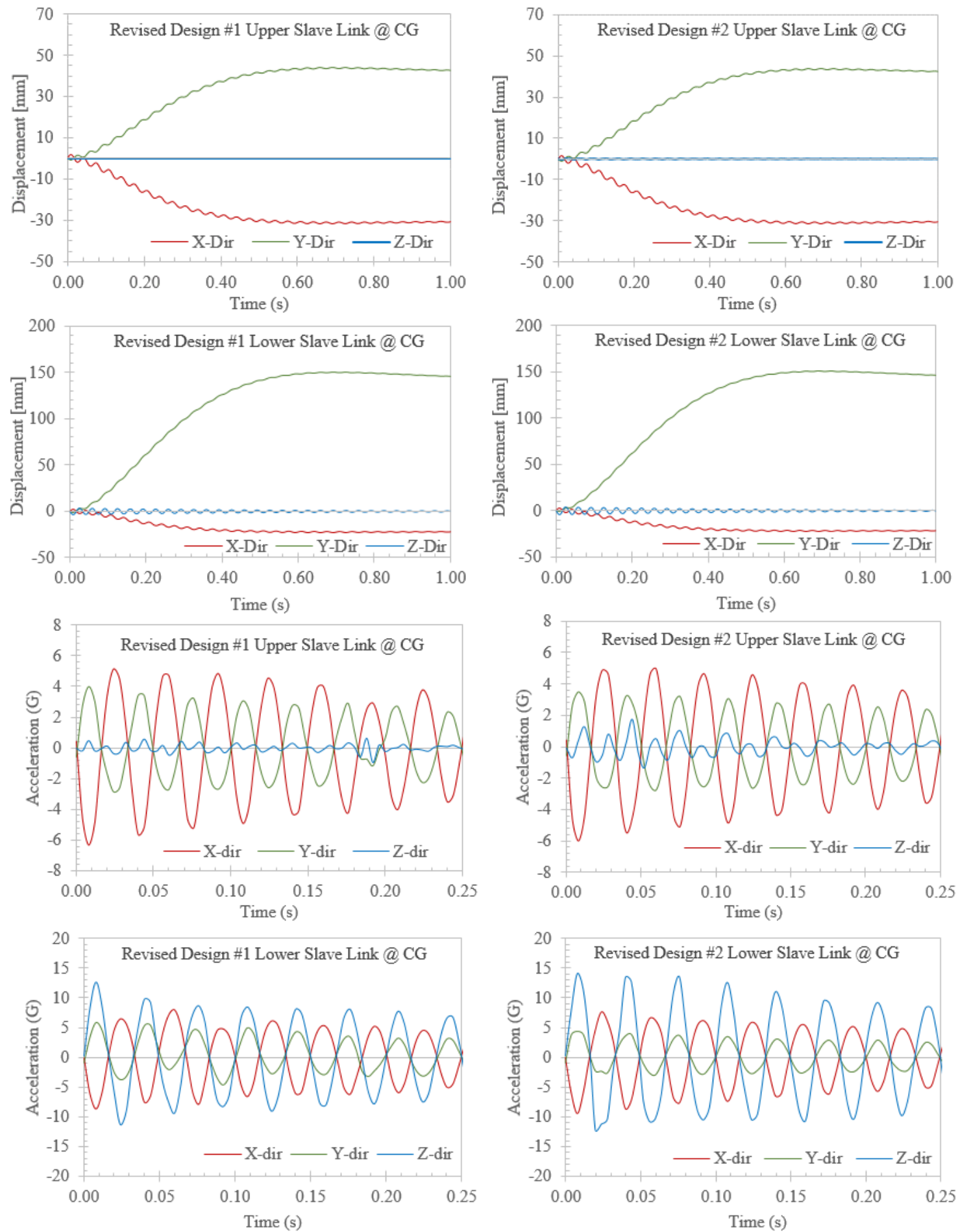

Figure 34 - Displacement and acceleration plots for RD\#1 and RD\#2. 
It can be seen the displacement and acceleration plots do not significantly differ from the baseline in Figure 23, with an overall variance less than $5 \mathrm{~mm}$ for displacements and $1 \mathrm{G}$ for acceleration. Components in RD\#1 do experience marginally lower acceleration values in the Z-

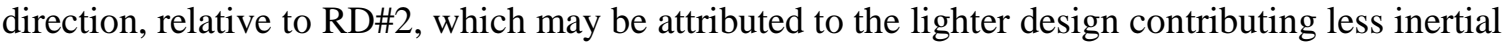
loading. Additionally, the presence of the double shear joint in the geometry of the upper slave link in $\mathrm{RD \# 1}$ versus the single shear joint in RD\#2 may also have an influence on this response. Even though lower Z-direction accelerations were observed, RD\#1 still saw higher stresses than RD\#2, as evident in Figure 36 and Figure 37. Overall, it should be noted that despite the differences in the approach to generate each design, the dynamic responses are quite similar.

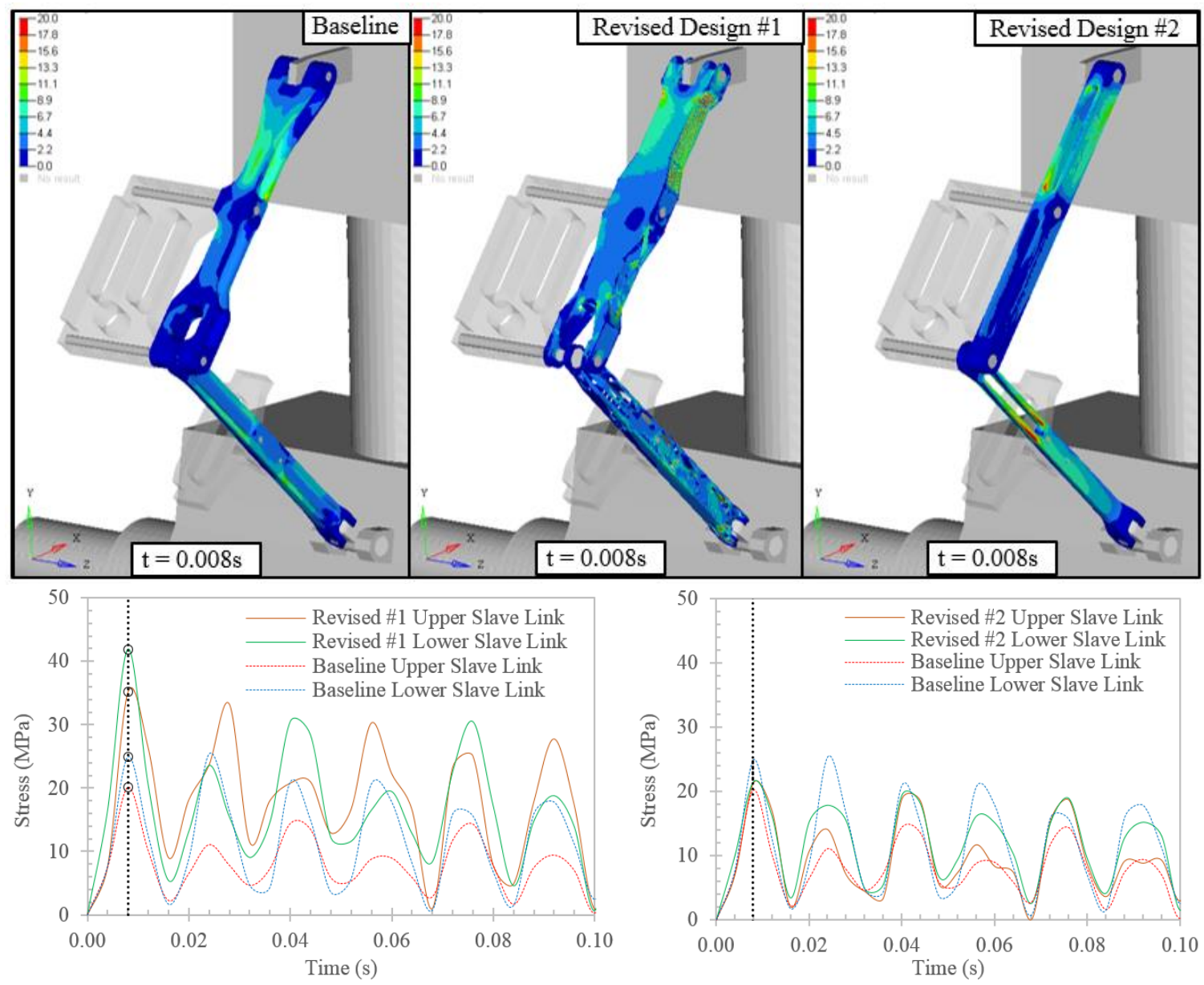

Figure 35 - Stress contours and plots comparison for revised and baseline designs. 


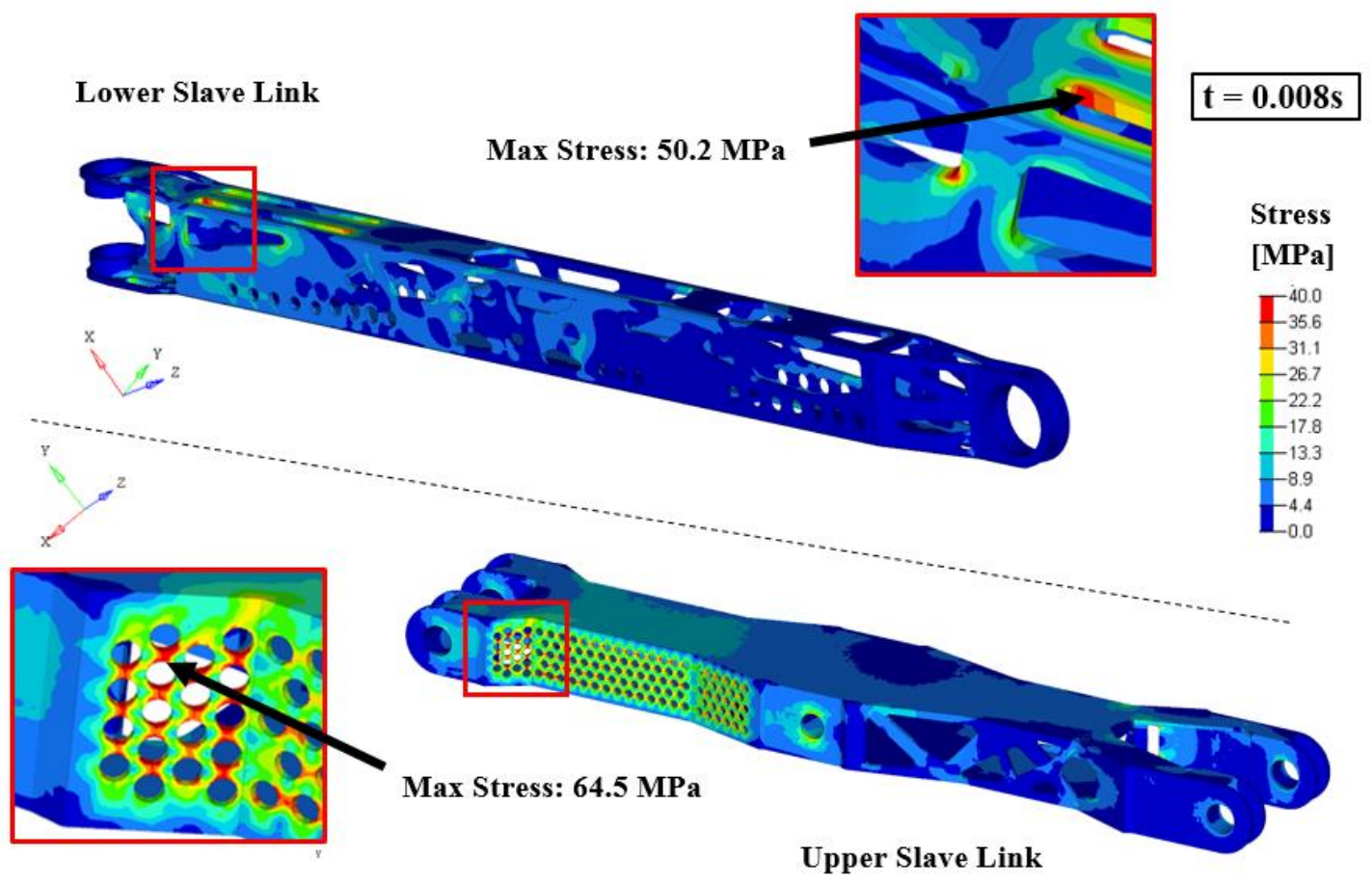

Figure 36 - Peak stress for components of RD\#1

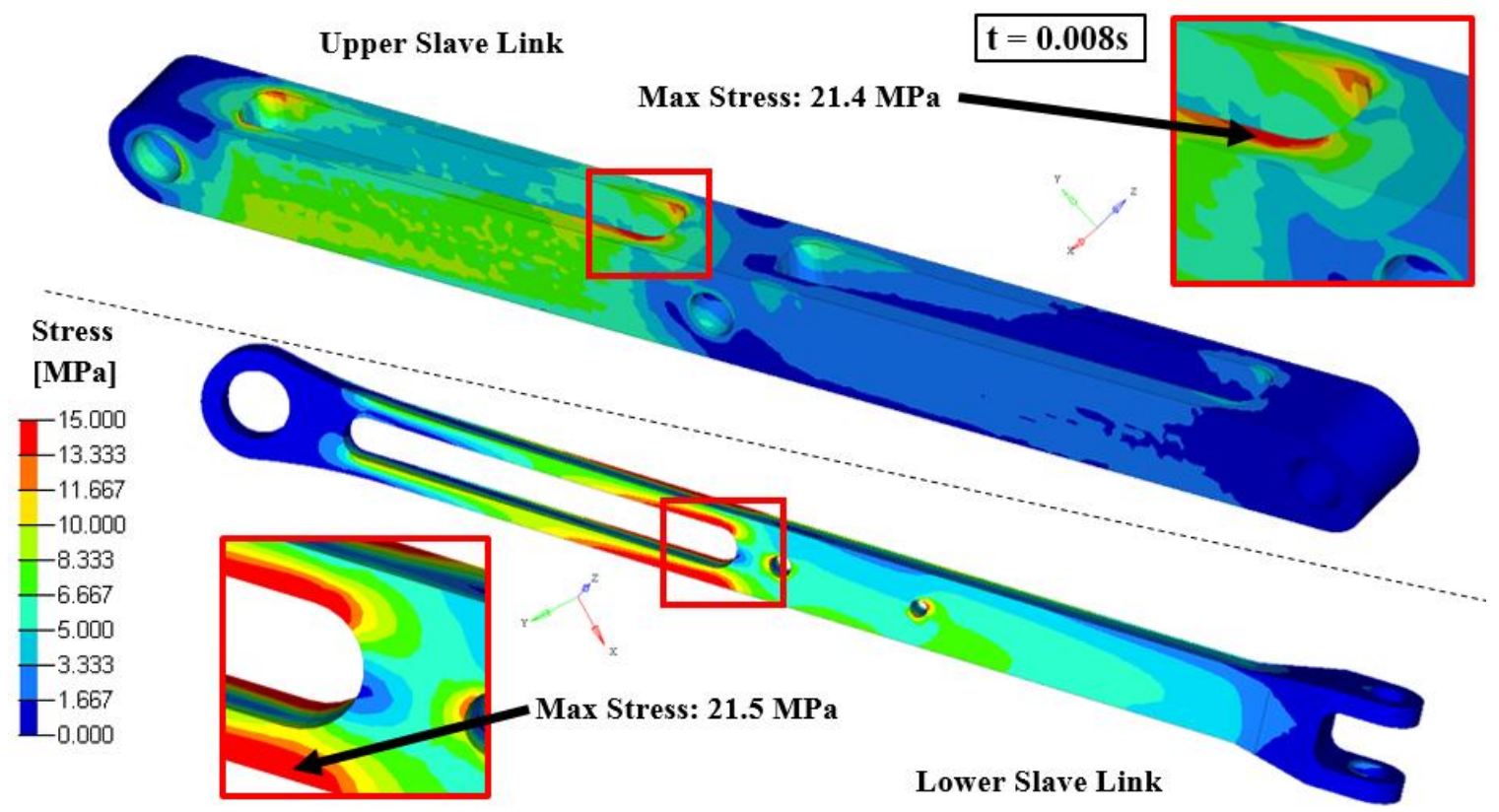

Figure 37 - Peak stress for components of RD\#2 
However, this trend was not observed in terms of the structural response. Figure 35 shows the two revised designs exhibiting a noticeable difference in terms of structural performance. The stresses experienced by RD\#1 was predominantly higher throughout the loading cycle when compared to the baseline. Alternatively, the stresses experienced by RD\#2 were at various instances either lower or similar to those observed in the baseline, despite being overall $36 \%$ lighter. This result justified the assumptions made for the upper slave link in the revised generation stage, and has consequently led to realized weight savings without compromising structural performance.

To closely examine and further investigate the cause of the stress concentrations, the peak stresses for RD\#1 shown in Figure 36 were identified, occurring in localized areas where cutouts or weight savings measures had been implemented. For the upper slave link, the high stresses occur in the perforated areas near the revolute joint (outlined in red), while the lower slave link experiences stress concentrations in a fillet close to the revolute joint (outlined in red). To reduce these peak stresses without significant impact on weight savings, size and shape optimization could be done as a subsequent step to further refine select features and dimensions in the design.

From Figure 37, the peak stresses for RD\#2 were identified near the center of each component and at the cable guides attachment. Similar to RD\#1, the peak stresses occurred on filleted features around the area identified. A probable cause for the induced stresses in this area may be due to the mass of the cable guides. Since material was removed from the slave link components to introduce weight savings, the inertial loading effects exerted by the cable guide onto the slave link are amplified. Despite this amplification, the stresses in RD\#2 were still comparable with those observed in the baseline design. 
Another important aspect to note is the difference in location where the peak stress occurs in the baseline design compared to the revised design. In the baseline, the peak stresses occur within the pinhole connection to the cable guide, while the revised designs experience their peak stresses away from the hole and along areas where thin features are present. A potential explanation for this result may be due to the change in overall compliance of the components as a result of lightweighting. This would consequently result in a decrease in stress near the pinhole because the revised designs generally has a lower cross sectional area than that of the baseline.

A summary of the performance metrics used to evaluate the revised designs are shown in Table 9.

Table 9 - Performance metrics of revised designs

\begin{tabular}{rccc}
\hline \multicolumn{1}{c}{ Component } & Weight Savings & Cost Savings & Peak Stress \\
\hline Revised Design \#1 & & & \\
Upper Slave Link & $-70 \%$ & N/A & $+74 \%$ \\
Lower Slave Link & $-60 \%$ & N/A & $+68 \%$ \\
Overall & $-67 \%$ & N/A & - \\
Revised Design \#2 & & & \\
Upper Slave Link & $-41 \%$ & $-37 \%$ & $+6 \%$ \\
Lower Slave Link & $-24 \%$ & $-3 \%$ & $-14 \%$ \\
Overall & $-36 \%$ & $(\sim 60 \% *)$ & - \\
\hline - Based on Safran's internal cost estimation tools. &
\end{tabular}




\subsection{Chapter 7: Discussions and Conclusions}

The research presented successfully demonstrated an effective and efficient methodology to computationally characterize, analyze, and optimize components of a landing gear assembly. First, the dynamic and structural behaviors were characterized for the slave link assembly under a worst-case landing load case. Second, a finite element model of the slave link assembly was created to perform MBD analysis for the dynamic and structural response of the system. Multiple MBD topology optimization runs with various settings were then performed on design spaces generated for each slave link component. Using the design insights gained from reinterpreting the optimal solutions, two revised designs were created with different approaches. The new designs were validated using the same model used to analyze the baseline design. RD\#1 achieved an overall weight savings of $67 \%$, peak stress increase of $74 \%$, and no apparent cost savings due to complex features present in the design. RD\#2 achieved an overall weight saving of $36 \%$, peak stress increase of $6 \%$, and an estimated $60 \%$ in cost savings.

It can be argued that the discrepancies between the calculated cost savings obtained from the manufacturing cost model developed in this research and Safran's cost estimation tool should be addressed. As mentioned in previous sections, there are numerous uncertainties and considerations within the manufacturing processes that make it challenging to predict; depending on the type of facilities and equipment available, the complexity of the cost model required may change significantly and may not be accurately captured by the techniques used. Although improvements to this aspect could be implemented, it should be noted that the emphasis for this research was on demonstrating the feasibility of utilizing MBD topology optimization for geometries and systems with greater complexity than simple test case problems.

Compared to traditional modeling and optimization approaches for dynamic systems, this methodology shows great potential for shortening product development cycles. Rather than performing manual translations of dynamic reaction forces, which are dependent on the geometry, 
into multiple static load cases for optimization, the utilization of ESLM for MBD topology optimization can enable users to perform this task efficiently. These time benefits can also be seen during the validation process, where new components were simply swapped into an existing MBD model and analyzed with minimal user inputs. Analogous with being a virtual test rig, another benefit that could be gained from this methodology is the ability to save costs in prototype iterations. The efficiency in setup and running multiple analysis for different designs helps identify and mitigate the risk of expensive design flaws later in the design process.

Although not performed in this research, it should be noted that the results generated from MBD analysis has the potential to be directly utilized in a fatigue analysis. The results generated from the MBD analysis may be readily exported as a dynamic load history file containing stress and strain data over time and read in a fatigue analysis software program such as nCode Design Life $\bigcirc$. The first advantage to this is the additional post-processing and computational resources required to export these results are minimal. Since it is possible to extract the load history directly from the MBD analysis, the results produced by the fatigue analysis has the potential to be more accurate than artificially scaling a static load history. This is under the assumption that the simulated conditions closely matches the behavior of the physical system.

The objective of this research was to demonstrate the potential of incorporating the methodology of MBD analysis and topology optimization with ESLM for aircraft landing gear assemblies, which undergo complex dynamic loading. Although manufacturability and shape/size optimization were not extensively covered, these items will be important to explore in future work, once this methodology has been accepted and adopted into modern design practices. If implemented, this methodology has the prospect of realizing additional cost and weight savings of various components and assemblies. As the future of the aerospace industry will inevitably lead to shorter development cycles and reduced prototyping iterations, it is important to continually develop and refine new design optimization methodologies to push the limits of design targets and performance. 


\subsection{References}

[1] International Air Transport Association, "IATA Forecasts Passenger Demand to Double Over 20 Years," IATA Press Release No. 59, no. October, pp. 18-22, 2016.

[2] S. Deveau, "Transportation - Should Airlines start charging by the pound," Financial Post, 2013.

[3] R. C. Fordham, "Airport Planning in the Context of the Third London Airport," Econ. J., vol. 80 , no. 3183, pp. 307-322, 1970.

[4] L. News, "Updating the A380 : the prospect of a neo version and what's involved," pp. $1-5,2014$.

[5] A. Kharina and D. Rutherford, "Fuel efficiency trends for new commercial jet aircraft: 1960 to 2014," Int. Counc. Clean Transp., no. August, 2015.

[6] A. France, "Checked Baggage Weight Limit," 2017. [Online]. Available: http://www.airfrance.com.br/BR/en/common/guidevoyageur/pratique/bagages-souteairfrance.htm.

[7] P. Hollinger, "Airlines bid to beat their weight problem," Financ. Times, 2016.

[8] J.-H. Zhu, W.-H. Zhang, and L. Xia, "Topology Optimization in Aircraft and Aerospace Structures Design,” Arch. Comput. Methods Eng., pp. 1-28, 2015.

[9] A. Engineering, "Multi-Disciplinary Design of an Aircraft Landing Gear with Altair HyperWorks Altair Engineering, October 2008,” no. October, 2008.

[10] G. Roloff, “Aircraft Landing Gear: The Evolution,” Airbus, no. April, p. 92, 2002.

[11] R. K. G. V. V. and S. R. P. Divakaran V.N., "Aircraft Landing Gear Design \& Development - How Advanced Technologies are helping to meet the challenges ?," Infosys (White Pap., 2015.

[12] B. Cox, “Cessna 210: High Wing Cruiser,” Cessna Own. Organ., 2015.

[13] Federal Aviation Authority, "Chapter 13 - Aircraft Landing Gear Systems," Aviat. Maint. Tech. Handb. - Airframe, pp. 20-22, 2014.

[14] Boeing, "Boeing 747 Landing gear," 2017. [Online]. Available: http://www.boeing.com/commercial/747.

[15] Boeing, "Boeing 777 Landing Gear," 2017. [Online]. Available: http://www.boeing.com/commercial/777/.

[16] Sukhoi, "Sukhoi-Superjet100 The evolutions of flight." [Online]. Available: http://www.superjetinternational.com/products/sukhoi-superjet100/overview/. 
[17] EASA, "Certification Specifications for Large Aeroplanes," Cs-25, no. September, p. 750, 2008.

[18] Tosaka, "Wheel arrangements of large airliners," 2010.

[19] H. P. Y. Hitch, “Aircraft Ground Dynamics,” Veh. Syst. Dyn., vol. 10, no. 4-5, pp. 319332, 1981.

[20] AGARD Report, The Design, Qualification and Maintenance of Vibration-Free Landing Gear (R-800), no. October. 1995.

[21] I. J. M. Besselink, Shimmy of Aircraft Main Landing Gears. Delft, Netherlands: Delft University of Technology, 2000.

[22] W. R. Krüger, I. J. M. Besselink, D. Cowling, D. B. Doan, W. Kortüm, and W. Krabacher, “Aircraft Landing Gear Dynamics: Simulation and Control," Veh. Syst. Dyn., vol. 28, no. 2-3, pp. 119-158, 1997.

[23] E. Denti and D. Fanteria, "Models of Wheel Contact Dynamics : An Analytical Study on the In-Plane Transient Responses of a Brush Model," Veh. Syst. Dyn., vol. 34, no. December 2012, pp. 37-41, 2010.

[24] N. Tadeusz, M. Jerzy, and B. Adam, "Numerical Analysis of a Front Support Landing Gear Dynamics,” pp. 3-10, 2006.

[25] J. Pritchard, "Overview of Landing Gear Dynamics," J. Aircr., vol. 38, no. 1, pp. 130137, 2001.

[26] W. R. Krüger and M. Morandini, "Recent developments at the numerical simulation of landing gear dynamics," CEAS Aeronaut. J., vol. 1, no. 1-4, pp. 55-68, 2014.

[27] T. Nguyen, A. Schonning, P. Eason, and D. Nicholson, "Methods for Analyzing Nose Gear During Landing Using Structural Finite Element Analysis," J. Aircr., vol. 49, no. 1, pp. 275-280, 2012.

[28] A. C. Gowda and N. B. S, "Trends in Mechanical Engineering \& Technology Linear Static and Fatigue Analysis of Nose Landing Gear for Trainer Aircraft,” vol. 4, no. 2, pp. $1-10,2014$.

[29] S. H. Oh, "A Study on Development of Dual Locking Linkage for Landing Gear for the Application to UAV," vol. 7, no. 2, pp. 41-48, 2014.

[30] J. A. C. Knowles, B. Krauskopf, and M. Lowenberg, "Numerical continuation analysis of a three-dimensional aircraft main landing gear mechanism," Nonlinear Dyn., vol. 71, no. 1-2, pp. 331-352, 2013.

[31] A. a Shabana, "Flexible multibody dynamics: review of past and recent developments," Multibody Syst. Dyn., vol. 1, pp. 189-222, 1997. 
[32] O. A. Bauchau, Flexible Multibody Dynamics, vol. 176. 2011.

[33] A. Cardona, "Superelements modelling in flexible multibody dynamics," Multibody Syst. Dyn., vol. 4, no. 2--3, pp. 245-266, 2000.

[34] M. Xingguo, Y. Xiaomei, and W. Bangchun, "Multi-body Dynamics Simulation on Flexible Crankshaft System," 12th IFTOMM World Congr. World Congr., pp. 3-5, 2007.

[35] D. G. Luenberger and Y. Ye, Linear and Nonlinear Programming, vol. 228, no. 9. 2016.

[36] K. Svanberg, "MMA and GCMMA - two methods for nonlinear optimization," vol. 1, pp. $1-15,2007$.

[37] A. Konak, D. W. Coit, and A. E. Smith, "Multi-objective optimization using genetic algorithms: A tutorial," Reliab. Eng. Syst. Saf., vol. 91, no. 9, pp. 992-1007, 2006.

[38] Y. Lian, A. Oyama, and M.-S. Liou, "Progress in Design Optimization Using Evolutionary Algorithms for Aerodynamic Problems," Prog. Aerosp. Sci., vol. 46, no. 56, pp. 199-223, 2010.

[39] O. Sigmund, "A 99 line topology optimization code written in MATLAB," Struct. Multidiscip. Optim., vol. 21, no. 2, pp. 120-127, 2001.

[40] M. P. Bendsøe and O. Sigmund, Topology optimization: theory, methods, and applications, vol. 2nd Editio, no. 724. 2003.

[41] L. Krog, A. Tucker, and G. Rollema, "Application of Topology, Sizing and Shape Optimization Methods To Optimal Design of Aircraft Components,” pp. 1-12, 2002.

[42] M. Tomlin and J. Meyer, "Topology Optimization of an Additive Layer Manufactured (ALM) Aerospace Part," 7th Altair CAE Technol. Conf. 2011, pp. 1-9, 2011.

[43] A. Remouchamps, M. Bruyneel, C. Fleury, and S. Grihon, "Application of a bi-level scheme including topology optimization to the design of an aircraft pylon," Struct. Multidiscip. Optim., vol. 44, no. 6, pp. 739-750, 2011.

[44] H. A. Lee and G. J. Park, “Topology Optimization for Structures With Nonlinear Behavior Using the Equivalent Static Loads Method," J. Mech. Des., vol. 134, no. 3, p. 14, 2012.

[45] W. S. Choi, K. B. Park, and G. J. Park, "Calculation of equivalent static loads and its application,” Nucl. Eng. Des., vol. 235, no. 22, pp. 2337-2348, 2005.

[46] G. J. Park, "Technical overview of the equivalent static loads method for non-linear static response structural optimization," Struct. Multidiscip. Optim., vol. 43, no. 3, pp. 319-337, 2011.

[47] B. S. Kang, W. S. Choi, and G. J. Park, "Structural optimization under equivalent static loads transformed from dynamic loads based on displacement," Comput. Struct., vol. 79, no. 2, pp. 145-154, 2001. 
[48] M. Li, W. Tang, and M. Yuan, "Structural dynamic topology optimization based on dynamic reliability using equivalent static loads," Struct. Multidiscip. Optim., vol. 49, no. 1, pp. 121-129, 2014.

[49] J. Sun, Q. Tian, and H. Hu, "Structural optimization of flexible components in a flexible multibody system modeled via ANCF," Mech. Mach. Theory, vol. 104, no. 29, pp. 59-80, 2016.

[50] M. Schmidt and K. Rohrbach, "ASM Handbook, Volume 1: Properties and Selection: Irons, Steels, and High-Performance Alloys," Met. Handb., vol. 1, pp. 793-800, 1990.

[51] DuPont, “Acetal Resin DuPont ${ }^{\mathrm{TM}}$ Delrin ${ }^{\circledR} 100$ NC010,” 2016.

[52] Altair OptiStruct, "OptiStruct 14.0 Reference Guide. Altair Engineering, Inc. Troy, MI," Altair Eng., 2015.

[53] K. E. Brenan, S. L. V. Campbell, and L. R. Petzold, "Numerical solution of initial-value problems in differential-algebraic equations," Book. p. 256, 1996.

[54] Altair OptiStruct, "MotionSolve 14.0 Reference Guide. Altair Engineering, Inc. Troy, MI," Altair Eng., 2015.

[55] A. OptiStruct, “OptiStruct 14.0 User Guide. Altair Engineering, Inc. Troy, MI,” Altair Eng., 2015.

[56] R. R. Craig and M. C. C. Bampton, "Coupling of Substructure for Dynamic Analyses," AIAA J., vol. 6, no. 7, pp. 1313-1319, 1968.

[57] B. B. Verma, J. D. Atkinson, and M. Kumar, "Study of fatigue behaviour of 7475 aluminium alloy,” Bull. Mater. Sci., vol. 24, no. 2, pp. 231-236, 2001.

[58] G. J. Park and B. S. Kang, "Validation of a structural optimization algorithm transforming dynamic loads into equivalent static loads," J. Optim. Theory Appl., vol. 118, no. 1, pp. 191-200, 2003.

[59] M. P. Bendsøe and O. Sigmund, "Material interpolation schemes in topology optimization,” Arch. Appl. Mech., vol. 69, no. 9-10, pp. 635-654, 1999.

[60] M. Zhou, Y. K. Shyy, and H. L. Thomas, "Checkerboard and minimum member size control in topology optimization," Struct. Multidiscip. Optim., vol. 21, no. 2, pp. 152-158, 2001.

[61] S. L. Vatanabe, T. N. Lippi, C. R. de Lima, G. H. Paulino, and E. C. N. Silva, "Topology optimization with manufacturing constraints: A unified projection-based approach," $A d v$. Eng. Softw., vol. 100, pp. 97-112, 2016. 


\subsection{Appendix}

\subsection{Appendix A - Sample Optimization Output File}

Due to the length of these output files, only a portion is shown to keep this section concise and still serve the purpose of explaining the functionality of the MATLAB code. Essentially, the code searches through the output file to find the iteration number (yellow) and extracts the corresponding compliance value (green).

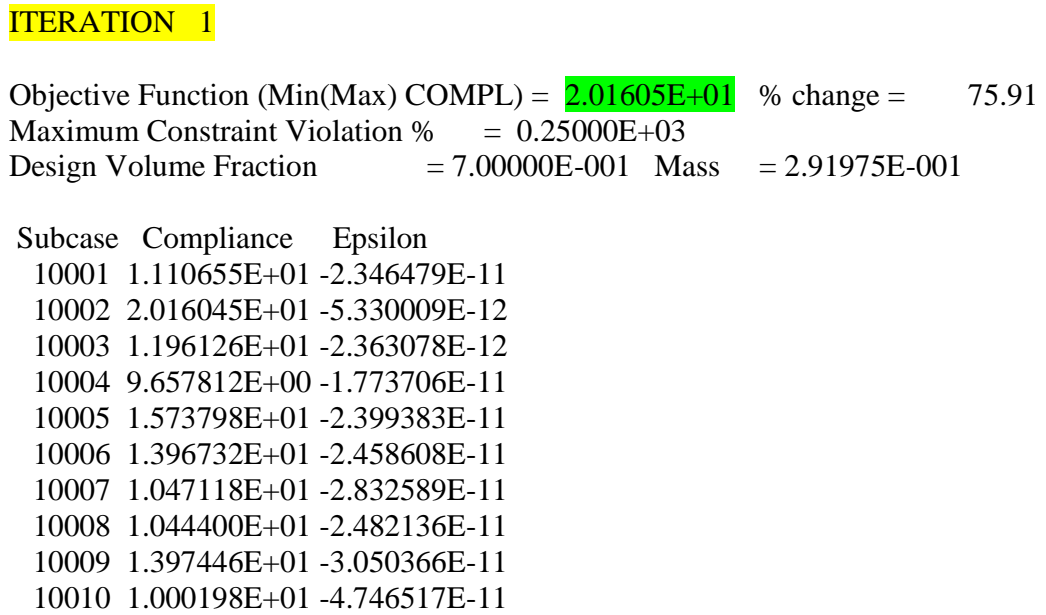

Note $:$ Epsilon $=$ Residual Strain Energy Ratio.

\section{RETAINED RESPONSES TABLE}

\begin{tabular}{|c|c|c|c|c|c|}
\hline $\begin{array}{l}\text { Response Type Response } \\
\text { User-ID Label } / \mathrm{R} \\
\\
\begin{array}{c}\text { /Model } \\
+ \text { Frqncy }\end{array}\end{array}$ & $\begin{array}{l}\text { se Subcase } \\
\text { /RANDPS El } \\
\text { 1 MID/PID/ } \\
\text { cy Mode No. }\end{array}$ & $\begin{array}{l}\text { Grid/ DOF } \\
\text { ement/ Comp } \\
\text { /Reg }\end{array}$ & $\begin{array}{l}\text { F/ Response } \\
\text { Value } \\
\text { Constraint } \\
\text { Bound }\end{array}$ & $\begin{array}{l}\text { Objective } \\
\text { Reference/ }\end{array}$ & $\begin{array}{l}\text { Viol. } \\
\%\end{array}$ \\
\hline 2 COMPL complian & 10001 & & & & OBJ \\
\hline 2 COMPL complian & 100 & -- TOTL & $2.016 \mathrm{E}+$ & / $1.000 \mathrm{E}+00$ & OBJ \\
\hline 2 COMPL complian & 10003 & -- TOTL & 1.196 & / 1.00 & OBJ \\
\hline 2 COMPL complian & 10004 & -- TOTL & $9.658 \mathrm{E}+00$ & / $1.000 \mathrm{E}+00$ & OBJ \\
\hline 2 COMPL complian & 10005 & -- TOTL & $1.574 \mathrm{E}+01$ & / $1.000 \mathrm{E}+00$ & OBJ \\
\hline 2 COMPL complian & 10006 & -- TOTL & $1.397 \mathrm{E}+01 /$ & / $1.000 \mathrm{E}+00$ & OBJ \\
\hline 2 COMPL complian & 10007 & -- TOTL & $1.047 \mathrm{E}+01$ & / $1.000 \mathrm{E}+00$ & OBJ \\
\hline 2 COMPL complian & 10008 & -- TOTL & $1.044 \mathrm{E}+01 /$ & / $1.000 \mathrm{E}+00$ & OBJ \\
\hline 2 COMPL complian & 10009 & -- TOTL & $1.397 \mathrm{E}+01$ & / $1.000 \mathrm{E}+00$ & OBJ \\
\hline 2 COMPL complian & 10010 & -- TOTL & $1.000 \mathrm{E}+01 /$ & / $1.000 \mathrm{E}+00$ & OBJ \\
\hline 1 VOLFR volfrac & $\begin{array}{ll}-- & 1\end{array}$ & SOLI 7.000 & $0 \mathrm{E}-01<2.0$ & $000 \mathrm{E}-01 \quad 250.0$ & \\
\hline
\end{tabular}

\section{MOST VIOLATED CONSTRAINTS TABLE}

Response Type Response Subcase Grid/ DOF/ Response Constraint Viol. User-ID Label /RANDPS Element/ Comp Value Bound \% /Model MID/PID/ /Reg

+Frqncy Mode No.

1 VOLFR volfrac $\quad--\quad 12$ SOLI $7.000 \mathrm{E}-01<2.000 \mathrm{E}-01250.0 \mathrm{~V}$ 


\subsection{Appendix B - MATLAB Output File Parser Code}

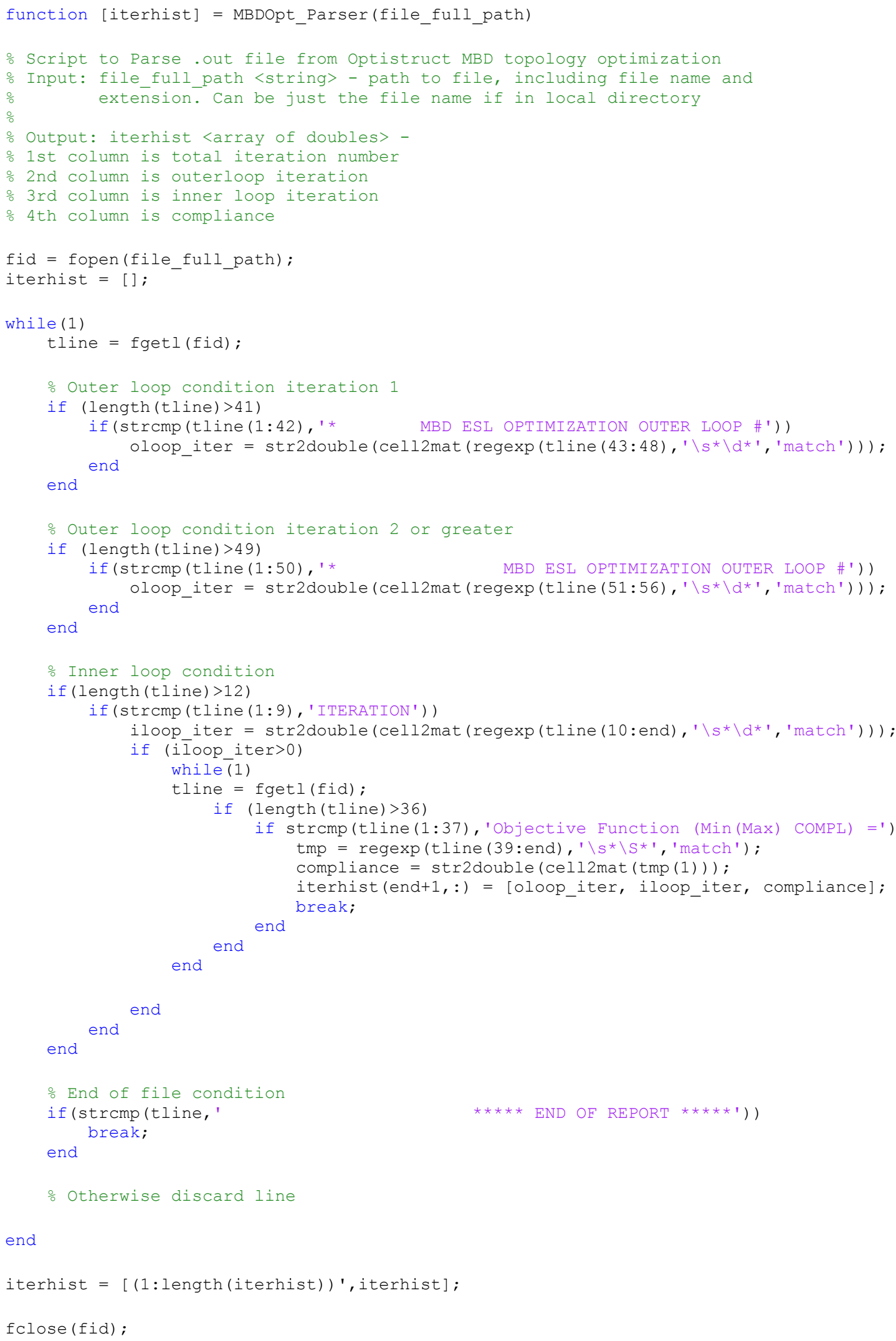




\subsection{Appendix C - Manufacturing Cost Analysis}

Table A-10 - Manufacturing cost analysis for upper slave link.

\begin{tabular}{|c|c|c|c|c|c|c|}
\hline Description & Weighting & USL-Baseline & USL-1a & USL-1b & USL-2a & USL-2b \\
\hline \multicolumn{7}{|c|}{ Performance Score } \\
\hline Weight Score & $100.00 \%$ & 1.000 & 0.783 & 0.700 & 0.631 & 0.591 \\
\hline Performance Score Total & & 1.000 & 0.783 & 0.700 & 0.631 & 0.591 \\
\hline \multicolumn{7}{|c|}{ Cost Score } \\
\hline Stock Material Score & $10.00 \%$ & 1.000 & 1.000 & 1.000 & 0.486 & 0.486 \\
\hline \# of Flips Score & $15.00 \%$ & 1.000 & 0.667 & 0.667 & 0.667 & 0.333 \\
\hline Material Removed Score & $50.00 \%$ & 1.000 & 1.233 & 1.323 & 0.680 & 0.769 \\
\hline Number of Milling Tools Score & $10.00 \%$ & 1.000 & 1.000 & 1.000 & 1.000 & 1.000 \\
\hline Number of Indidivual Radii Score & $5.00 \%$ & 1.000 & 1.000 & 1.000 & 0.667 & 0.667 \\
\hline Number of Indidivual Pockets Score & $5.00 \%$ & 1.000 & 0.500 & 0.833 & 0.667 & 0.333 \\
\hline Number of External Contours Score & $5.00 \%$ & 1.000 & 0.000 & 0.000 & 0.000 & 0.000 \\
\hline Cost Score Total & $100.00 \%$ & 1.000 & 0.992 & 1.053 & 0.655 & 0.633 \\
\hline
\end{tabular}

Lower score is better 
Table A-11 - Manufacturing cost analysis for upper slave link.

\begin{tabular}{|c|c|c|c|c|c|}
\hline Description & Units & LSL-Baseline & LSL-1 & LSL-2a & LSL-2b \\
\hline \multicolumn{6}{|c|}{ Performance Score } \\
\hline Weight Score & $100.00 \%$ & 1.000 & 1.065 & 0.683 & 0.756 \\
\hline Performance Score Total & & 1.000 & 1.065 & 0.683 & 0.756 \\
\hline \multicolumn{6}{|c|}{ Cost Score } \\
\hline Stock Material Score & $10.00 \%$ & 1.000 & 1.000 & 1.000 & 1.000 \\
\hline \# of Flips Score & $15.00 \%$ & 1.000 & 0.750 & 0.750 & 0.750 \\
\hline Material Removed Score & $50.00 \%$ & 1.000 & 0.977 & 1.111 & 1.086 \\
\hline Number of Milling Tools Score & $10.00 \%$ & 1.000 & 1.000 & 1.000 & 1.000 \\
\hline Number of Indidivual Radii Score & $5.00 \%$ & 1.000 & 1.000 & 1.000 & 1.000 \\
\hline Number of Indidivual Pockets Score & $5.00 \%$ & 1.000 & 0.600 & 0.600 & 0.400 \\
\hline Number of External Contours Score & $5.00 \%$ & 1.000 & 1.000 & 1.000 & 1.000 \\
\hline Cost Score Total & $100.00 \%$ & 1.000 & 0.931 & 0.998 & 0.975 \\
\hline
\end{tabular}

Lower score is better 


\subsection{Appendix D - Revised Design \#2 Drawings}

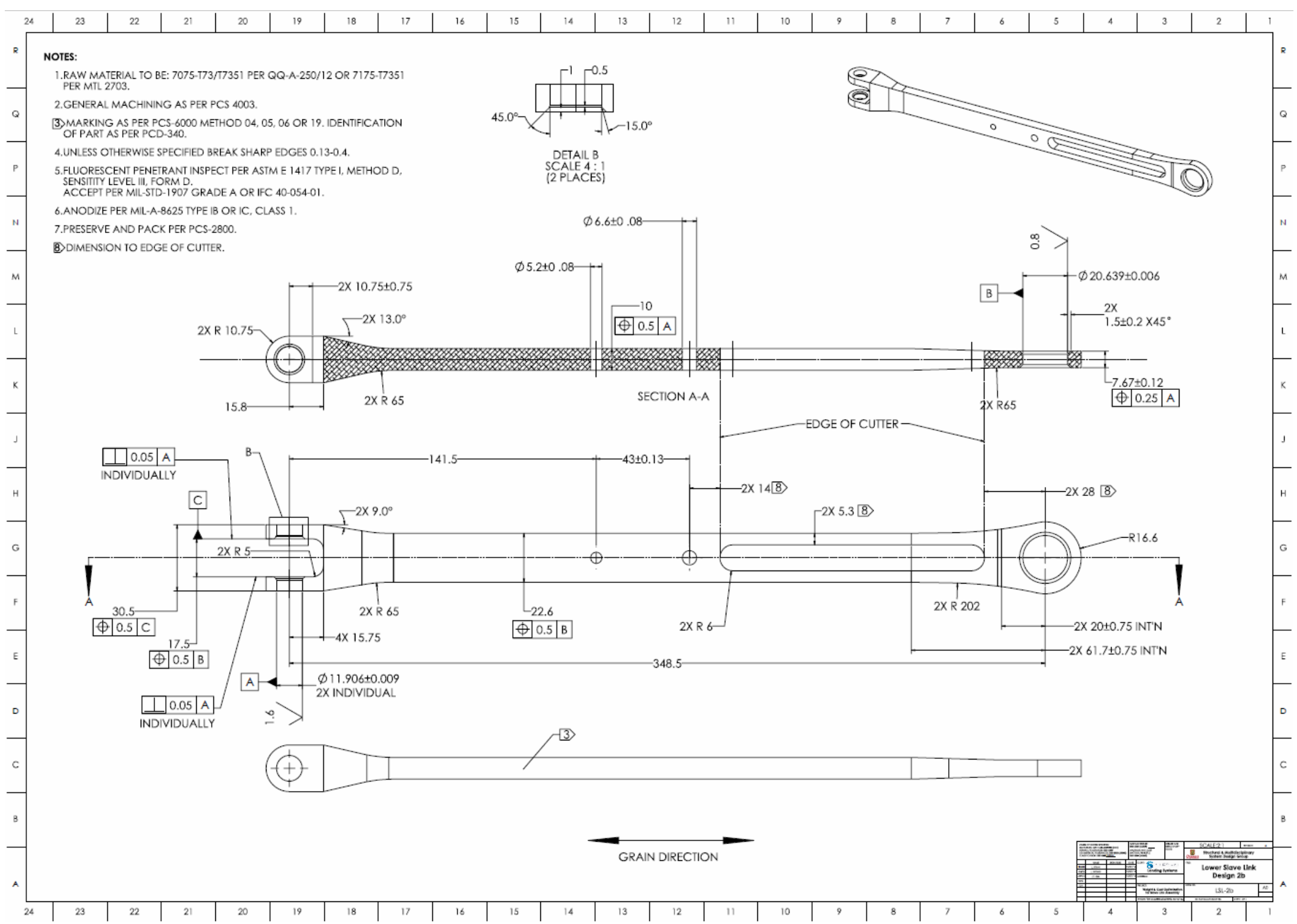

Figure A-38 - Revised Design \#2 lower slave link detailed drawing. 


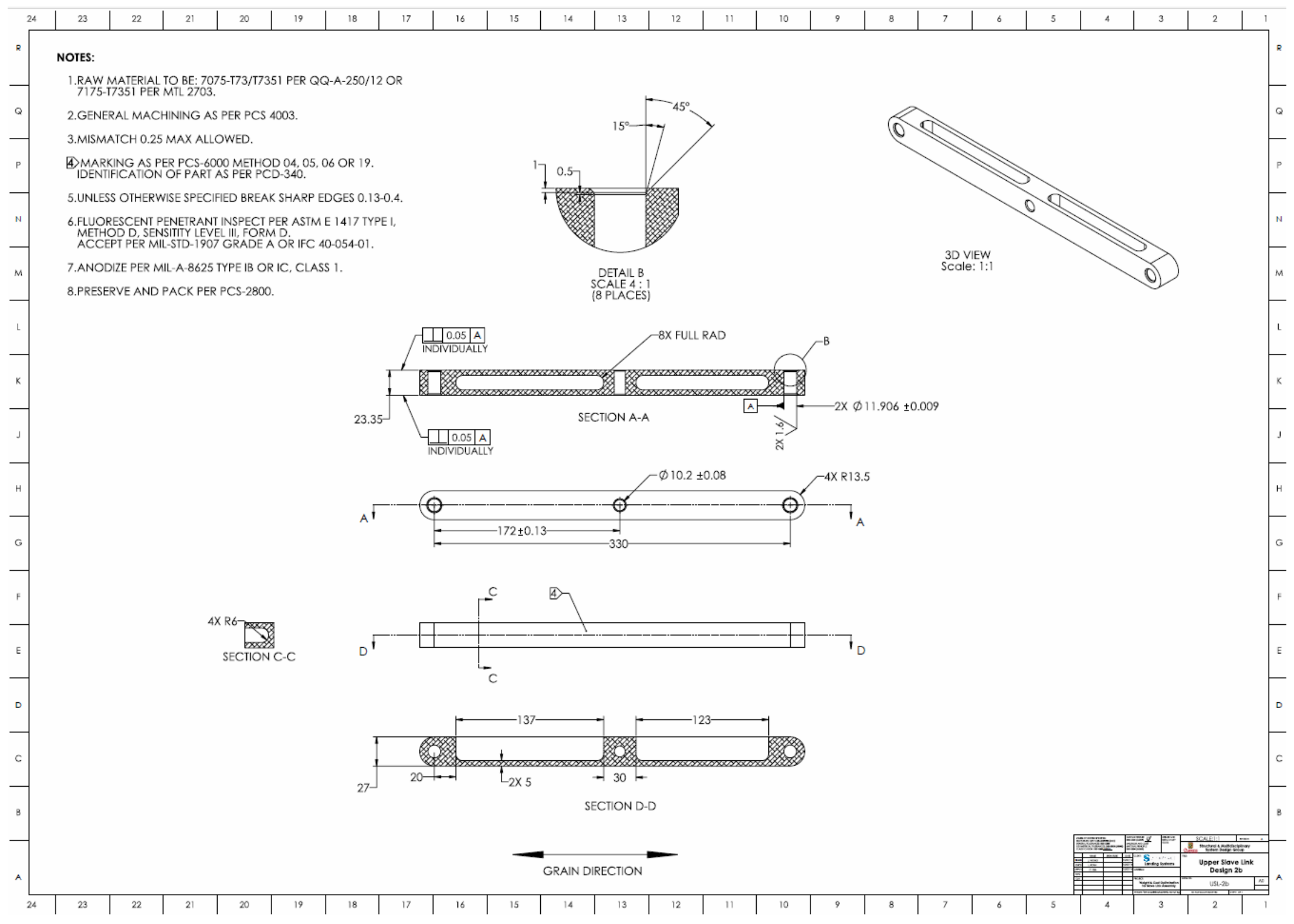

Figure A-39 - Revised Design \#2 upper slave link detailed drawing. 